Olivier Glass

\title{
On the controllability of the 1-D isentropic Euler equation
}

Received October 4, 2005 and in revised form February 23, 2006

\begin{abstract}
We study the controllability problem for the one-dimensional Euler isentropic system, both in Eulerian and Lagrangian coordinates, by means of boundary controls, in the context of weak entropy solutions. We give a sufficient condition on the initial and final states under which the first one can be steered to the latter.
\end{abstract}

Keywords. Controllability, isentropic Euler equations, hyperbolic systems of conservation laws, entropy solutions, front-tracking algorithm

\section{Introduction}

\subsection{The problem}

In this paper, we study the problem of controllability of the isentropic compressible Euler equation in one space variable. The equation under consideration, when written in Eulerian coordinates, is the following system:

$$
\left\{\begin{array}{l}
\partial_{t} \rho+\partial_{x}(m)=0 \\
\partial_{t}(m)+\partial_{x}\left(m^{2} / \rho+\kappa \rho^{\gamma}\right)=0 .
\end{array}\right.
$$

In this system, $\rho=\rho(t, x) \geq 0$ describes the local density of the fluid at time $t$ and position $x \in[0,1]$, and $m(t, x)$ its momentum (that is to say, $v(t, x)=m(t, x) / \rho(t, x)$ is the local velocity of the fluid). The first of these two equations describes the conservation of mass, whereas the second one describes the conservation of momentum, when the pressure is given by the following polytropic law: $p(\rho)=\kappa \rho^{\gamma}$, with $\gamma \in(1,3]$ and $\kappa>0$.

We are also interested in the system written in Lagrangian coordinates:

$$
\left\{\begin{array}{l}
\partial_{t} \tau-\partial_{x} v=0 \\
\partial_{t} v+\partial_{x}\left(\kappa \tau^{-\gamma}\right)=0
\end{array}\right.
$$

Here $\tau:=1 / \rho$ is the specific volume. As proven in [35], solutions of (EI) and of (P) are equivalent via a suitable change of coordinates, even in the case of weak (entropy)

O. Glass: Université Pierre et Marie Curie-Paris 6, UMR 7598 Laboratoire Jacques-Louis Lions, F-75005 Paris, France; e-mail: glass@ ann.jussieu.fr

Mathematics Subject Classification (2000): 93B05, 35B37, 35L65 
solutions. However, the controllability problems described below are different, since they occur in the fixed space domain $[0,1]$.

It is well-known that such equations are hyperbolic systems of conservation laws, in which singularities may appear in finite time even if the initial condition is smooth. Hence it is natural to consider weak solutions, which satisfy additional "entropy conditions" aimed at singling out the physically relevant solution.

When we fix an initial condition, $(\mathrm{EI})$ and $(\mathrm{P})$ are underdetermined because we have not prescribed boundary conditions. In this paper, the boundary conditions are not given in advance and are considered as a control, that is, a way to influence the system in order to make it reach a given state. In this framework, the controllability problem that we consider is the following: given suitable states $u_{0}$ and $u_{1}$ of the system, is it possible to find an entropy solution of $(\mathrm{EI})$ (resp. $(\mathrm{P})$ ) defined for a time interval $[0, T]$, such that

$$
u_{\mid t=0}=u_{0} \quad \text { and } \quad u_{\mid t=T}=u_{1} \text { ? }
$$

The nature of the system suggests that one has to require additional conditions on the states $u_{0}$ and $u_{1}$ (particularly the latter) in order to get a positive answer. Here we give sufficient conditions on $u_{0}$ and $u_{1}$ in order that the above problem has a solution. Note however that the conditions below are not necessary in general.

\subsection{Mathematical setting}

Let us define more precisely the type of solutions that we consider. We restrict ourselves to the case of solutions that are in $B V([0,1])$ for all time, and which do not meet the vacuum (moreover, we will restrict to the case when $u_{0}$ and $u_{1}$ have small total variation). Let us remark that existence theory for isentropic gas dynamics is established in the much more general framework of $L^{\infty}$ solutions that can contain vacuum (see [29]).

Both systems $(\mathrm{EI})$ and $(\mathrm{P})$ are written in the form

$$
u_{t}+f(u)_{x}=0,
$$

for $u=(\rho, m)$ and $u=(\tau, v)$ respectively. In this paper, the state $u$ belongs to $\mathbb{R}^{+*} \times \mathbb{R}$ (hence no vacuum is present).

Recall that an entropy/entropy flux couple is defined as a couple $(\eta, q)$ of regular functions satisfying

$$
\forall u \in \mathbb{R}^{+*} \times \mathbb{R}, \quad D \eta(u) \cdot D f(u)=D q(u) .
$$

Then we have the following definition:

Definition 1. A function $u \in L^{\infty}\left([0, T] ; B V\left([0,1] ; \mathbb{R}^{+*} \times \mathbb{R}\right)\right) \cap \operatorname{Lip}\left([0, T] ; L^{1}([0,1]\right.$; $\left.\mathbb{R}^{+*} \times \mathbb{R}\right)$ ) is called a weak solution of $(\mathrm{EI})$ or $(\mathrm{P})$ when it satisfies $(\mathrm{EI})$ or $(\mathrm{P})$ in the distribution sense:

$\forall \varphi \in \mathcal{D}([0, T) \times(0,1))$,

$\int_{[0,+\infty) \times(0,1)}\left(u(t, x) \varphi_{t}(t, x)+f(u)(t, x) \varphi_{x}(t, x)\right) d t d x+\int_{[0,1]} \varphi(x) u_{0}(x) d x=0$. 
It is called an entropy solution when moreover, for any entropy/entropy flux couple $(\eta, q)$ with $\eta$ convex, one has in the sense of measures

$$
\eta(u)_{t}+q(u)_{x} \leq 0
$$

that is,

$$
\begin{aligned}
& \forall \varphi \in \mathcal{D}((0, T) \times(0,1)) \text { with } \varphi \geq 0, \\
& \qquad \int_{(0,+\infty) \times(0,1)}\left(\eta(u(t, x)) \varphi_{t}(t, x)+q(u(t, x)) \varphi_{x}(t, x)\right) d t d x \geq 0 .
\end{aligned}
$$

Now the problem is: given $u_{0}$ and $u_{1}$ in $B V([0,1])$ (with small total variation), does there exist $T>0$ and an entropy solution defined in $[0, T] \times[0,1]$ such that $(1]$ is satisfied?

Let us remark that here we do not prescribe the time of controllability in advance; it strongly depends on the states $u_{0}$ and $u_{1}$ considered.

We emphasize that it is more convenient to work with the underdetermined system, without looking for the control explicitly. It could be retrieved from the traces of the solution on the boundary. Note however that for such systems, one cannot impose Dirichlet boundary conditions, but rather "entering" boundary conditions as described in [18]. For the study of the initial boundary problem, we refer for instance to [2].

\subsection{Results}

Fix $c_{\gamma}:=(1 / 2+(\gamma-1) / 4 \sqrt{\gamma})^{-1}$. We define pairs of Riemann invariants for the systems (EI) and (P) as follows:

$$
w^{1}(u)=\frac{m}{\rho}+\frac{2 \sqrt{\kappa \gamma}}{\gamma-1} \rho^{(\gamma-1) / 2} \quad \text { and } \quad w^{2}(u)=\frac{m}{\rho}-\frac{2 \sqrt{\kappa \gamma}}{\gamma-1} \rho^{(\gamma-1) / 2}
$$

for $u=(\rho, m)$, and

$$
w^{1}=v+\frac{2 \sqrt{\kappa \gamma}}{\gamma-1} \tau^{-(\gamma-1) / 2} \quad \text { and } \quad w^{2}=v-\frac{2 \sqrt{\kappa \gamma}}{\gamma-1} \tau^{-(\gamma-1) / 2}
$$

for $u=(\tau, v)$, respectively. We denote the characteristic speeds of the systems by $\lambda_{1}$ and $\lambda_{2}$.

The results that we prove in this paper are the following: concerning the system (EI):

Theorem 1. Let $\bar{u}_{0}$ and $\bar{u}_{1}$ be two constant states in $\mathbb{R}^{+*} \times \mathbb{R}$. Set $\bar{\lambda}_{1}:=\lambda_{1}\left(\bar{u}_{1}\right)$ and $\bar{\lambda}_{2}:=\lambda_{2}\left(\bar{u}_{1}\right)$. For any $\alpha \in(0,1)$, there exist $\varepsilon_{1}=\varepsilon_{1}\left(\bar{u}_{0}\right)>0, \varepsilon_{2}=\varepsilon_{2}\left(\bar{u}_{1}, \alpha\right)>0$, and $T=T\left(\bar{u}_{0}, \bar{u}_{1}\right)>0$ such that, for any $u_{0}, u_{1} \in B V\left([0,1] ; \mathbb{R}^{+*} \times \mathbb{R}\right)$ satisfying:

$$
\begin{aligned}
& \left\|u_{0}-\bar{u}_{0}\right\| \leq \varepsilon_{1} \quad \text { and } \quad T V\left(u_{0}\right) \leq \varepsilon_{1}, \\
& \left\|u_{1}-\bar{u}_{1}\right\| \leq \varepsilon_{2} \quad \text { and } \quad T V\left(u_{1}\right) \leq \varepsilon_{2},
\end{aligned}
$$

and 


$$
\begin{aligned}
& \forall x, y \in[0,1], x<y, \\
& \qquad \frac{w^{2}\left(u_{1}(x)\right)-w^{2}\left(u_{1}(y)\right)}{x-y} \leq c_{\gamma}(1-\alpha) \max \left(\frac{\bar{\lambda}_{2}-\bar{\lambda}_{1}}{1-y}, \frac{\bar{\lambda}_{1}}{x}, \frac{-\bar{\lambda}_{1}}{1-y}\right),
\end{aligned}
$$

$\forall x, y \in[0,1], x<y$,

$$
\frac{w^{1}\left(u_{1}(x)\right)-w^{1}\left(u_{1}(y)\right)}{x-y} \leq c_{\gamma}(1-\alpha) \max \left(\frac{\bar{\lambda}_{2}-\bar{\lambda}_{1}}{x}, \frac{-\bar{\lambda}_{2}}{1-y}, \frac{\bar{\lambda}_{2}}{x}\right),
$$

there is an entropic solution $u$ of $(\mathrm{EI})$ in $[0, T] \times[0,1]$ such that

$$
\begin{aligned}
& u_{\mid t=0}=u_{0}, \\
& u_{\mid t=T}=u_{1} .
\end{aligned}
$$

For the system $(\mathrm{P})$ in Lagrangian coordinates, we have:

Theorem 2. Let $\bar{u}_{0}$ and $\bar{u}_{1}$ be two constant states in $\mathbb{R}^{+*} \times \mathbb{R}$. Set $\bar{\lambda}_{1}:=\lambda_{1}\left(\bar{u}_{1}\right), \bar{\lambda}_{2}:=$ $\lambda_{2}\left(\bar{u}_{1}\right)$, and

$$
\bar{\xi}_{1}:=\frac{\partial \lambda_{1}}{\partial w^{2}}\left(\bar{u}_{1}\right) \text { and } \quad \bar{\xi}_{2}:=\frac{\partial \lambda_{2}}{\partial w^{1}}\left(\bar{u}_{1}\right) .
$$

For any $\alpha \in(0,1)$, there exist $\varepsilon_{1}=\varepsilon_{1}\left(\bar{u}_{0}\right)>0, \varepsilon_{2}=\varepsilon_{2}\left(\bar{u}_{1}, \alpha\right)>0$, and $T=$ $T\left(\bar{u}_{0}, \bar{u}_{1}\right)>0$ such that, for any $u_{0}, u_{1} \in B V([0,1])$ satisfying:

$$
\begin{aligned}
& \left\|u_{0}-\bar{u}_{0}\right\| \leq \varepsilon_{1} \quad \text { and } \quad T V\left(u_{0}\right) \leq \varepsilon_{1}, \\
& \left\|u_{1}-\bar{u}_{1}\right\| \leq \varepsilon_{2} \quad \text { and } \quad T V\left(u_{1}\right) \leq \varepsilon_{2},
\end{aligned}
$$

and

$$
\begin{aligned}
& \forall x, y \in[0,1], x<y, \quad \frac{w^{2}\left(u_{1}(x)\right)-w^{2}\left(u_{1}(y)\right)}{x-y} \leq(1-\alpha) \frac{1}{\bar{\xi}_{1}} \frac{\bar{\lambda}_{2}-\bar{\lambda}_{1}}{1-y}, \\
& \forall x, y \in[0,1], x<y, \quad \frac{w^{1}\left(u_{1}(x)\right)-w^{1}\left(u_{1}(y)\right)}{x-y} \leq(1-\alpha) \frac{1}{\bar{\xi}_{2}} \frac{\bar{\lambda}_{2}-\bar{\lambda}_{1}}{x},
\end{aligned}
$$

there is an entropic solution $u$ of $(\bar{P})$ in $[0, T] \times[0,1]$ such that

$$
\begin{aligned}
& u_{\mid t=0}=u_{0}, \\
& u_{\mid t=T}=u_{1} .
\end{aligned}
$$

Remark 1. Let us remark that the Oleĭnik-type conditions $(10)-(11)$ and $(16-(17)$ are not satisfied for general trajectories of the systems $(\mathrm{EI})$ and $(\mathrm{P})$ (the interaction of two shocks of the same family just before the final time generates waves in which the ratios considered in $(10)-(11)$ and $(16)-(17)$ can be arbitrarily large). See for instance [8, Subsection 10.2].

Remark 2. Let us also remark that the right hand sides of $(10),(11),(16)$ and $(17)$ are always positive. In particular, constant states can always be reached. 


\subsection{Previous studies and comments}

Let us recall some results obtained in the field of controllability of one-dimensional hyperbolic systems of conservations laws. In the case of regular (that is, $C^{1}$ ) solutions, it was shown by $\mathrm{Li}$ and Rao (see [28]) that for a quasilinear hyperbolic equation in which characteristic speeds are bounded away from 0 , it is possible to connect two states with small $C^{1}$ norm. Let us remark also that for $(\mathrm{EI})$ the particular case $\gamma=2$ corresponds to the Saint-Venant (or shallow-water) equation, for which several controllability problems have been considered in the framework of $C^{1}$ solutions (see for instance [16, 23]).

Concerning weak entropy solutions, the control of convex scalar conservation laws has been studied by Ancona and Marson [5], who completely describe the states attainable starting from $u_{0}=0$. In [24], Horsin considers the case $u_{0} \neq 0$ for Burgers' equation, by using the return method, which was introduced by Coron in [14] and is also a key ingredient here.

The study of controllability problems for weak entropy of systems of conservation laws has been initiated by Bressan and Coclite in [9]. For general strictly hyperbolic systems of conservation laws with genuinely nonlinear or linearly degenerate fields (in the sense of Lax [25]), and characteristic speeds bounded away from 0 , it is shown that one can drive a small $B V$ state to a constant state, asymptotically in time, by an open-loop control. For the problem of controllability in finite time, they prove the following negative result for a class of systems containing the system below (which was introduced by Di Perna [17]), and which is somewhat close to (EI):

$$
\left\{\begin{array}{l}
\partial_{t} \rho+\partial_{x}(\rho u)=0 \\
\partial_{t} u+\partial_{x}\left(\frac{u^{2}}{2}+\frac{K^{2}}{\gamma-1} \rho^{\gamma-1}\right)=0 .
\end{array}\right.
$$

Theorem 2 in [9] proves that there are initial conditions, with arbitrarily small total variation, for which no entropy solution which has small total variation for any $t$ can reach a constant state. The system (DP) has the feature that the interaction of two shocks of a family generates a shock in the other family. Note that for $(\overline{E I})$, on the contrary, the interaction of two shocks of a family generates a rarefaction wave in the other family. However, [9] gives strong indications that controlling (EI) with the solution of total variation of the same order as $T V\left(u_{0}\right)$ for all times would be a strongly nontrivial matter (one can even doubt that this is possible). Here we use the "return method" which consists in strongly perturbing the system in order to achieve controllability. This suggests distinguishing two types of controllability properties: a controllability property for which one should expect the size of the control to be of the same order as the distance from $u_{0}$ to $u_{1}$, and controllability via strong perturbations in which the control can be large with respect to the distance to achieve. In the present paper, the latter is considered; however, one can construct a solution with small total variation $\tau$ connecting states that are of small total variation $\varepsilon$, but $\varepsilon$ is very small with respect to $\tau$ (clearly not of the same order) - see Subsection 1.5 below. It is, of course, rather unsatisfactory that, despite the fact that we consider solutions which may have large total variation, the result applies only to initial and final states with small total variation. 
Finally, in [4], Ancona and Coclite investigate the controllability properties for the Temple class systems (see [33] for a precise definition). They prove that the controllability applies in the case of characteristic speeds strictly separated from 0 and Oleĭnik-type inequalities on $u_{1}$. An important difference with the results here is that no small total variation is assumed (the solutions are even considered in $L^{\infty}$ ), and the Oleĭnik conditions imposed on $u_{1}$ are actually satisfied by the trajectories of the system (with perhaps a different multiplicative constant). Parts of the construction here are analogous to those of [4] and [9].

\subsection{Additional remarks}

Two different methods are developed in this paper for the systems $(\mathrm{EI})$ and $(\mathrm{P})$, respectively. It should be noted that, while the first method cannot apply to $(\mathrm{P})$, the method developed in Section 6 could be used for the system (EI), if the reference state $\bar{u}_{0}$ satisfies $\lambda_{1}\left(\bar{u}_{0}\right)<0<\lambda_{2}\left(\bar{u}_{0}\right)$. This is due to the fact that the first method relies on the possibility to shift the sign of the characteristic speeds, while the second one relies on the possibility to generate rarefaction waves (and hence cancelations) via interaction of shocks of the same family.

Also, the following fact will appear during the proofs: for the system (P) and for (EI) when moreover $\lambda_{1}\left(\bar{u}_{0}\right)<0<\lambda_{2}\left(\bar{u}_{0}\right)$, one can use the second method to get solutions with small total variation $\tau$ (for all time), for states $u_{0}$ and $u_{1}$ sufficiently close to $\bar{u}_{0}$ and $\bar{u}_{1}$ in $B V$, but the radii of the corresponding balls in $B V$ (that is, $\varepsilon_{1}$ and $\varepsilon_{2}$ in Theorems 1 and 2 are very small with respect to $\tau$ (in a nonlinear way). Also, the time of controllability could grow as $\tau \rightarrow 0^{+}$. For the system [EI] when either $\lambda_{1}\left(\bar{u}_{0}\right)<\lambda_{2}\left(\bar{u}_{0}\right) \leq 0$ or $0 \leq \lambda_{1}\left(\bar{u}_{0}\right)<\lambda_{2}\left(\bar{u}_{0}\right)$, one can use the first method to obtain solutions with small total variation $\tau$ (again if $T V\left(u_{0}\right)$ and $T V\left(u_{1}\right)$ are very small with respect to $\tau$ ).

\subsection{Structure of the paper}

Theorem 1 is proven in Sections 2 to 5 In Section 2, we introduce some preliminaries and notations. The general method is described in Subsection 2.4 The proof of the result is in three steps developed respectively in Sections 3, 4, 5. The first step in the case of Theorem 2 is completely different and is described in Section 6.

\section{Preliminaries and notations}

\subsection{Characteristics of the system}

Written as an equation for $u=(\rho, m)$, the system (EI) is a system of conservations laws, which is strictly hyperbolic as long as $\rho>0$, that is,

$$
\lambda_{1}(u)<\lambda_{2}(u)
$$


where $\lambda_{1}$ and $\lambda_{2}$ given by

$$
\lambda_{1}=\frac{m}{\rho}-\sqrt{\kappa \gamma} \rho^{(\gamma-1) / 2} \quad \text { and } \quad \lambda_{2}=\frac{m}{\rho}+\sqrt{\kappa \gamma} \rho^{(\gamma-1) / 2}
$$

are the two (real) eigenvalues of the Jacobian matrix $A$ associated with the flux function $f$ :

$f(\rho, m)=\left(\begin{array}{c}m \\ m^{2} / \rho+\kappa \rho^{\gamma}\end{array}\right) \quad$ and $\quad A(\rho, m)=\left(\begin{array}{cc}0 & 1 \\ -m^{2} / \rho^{2}+\gamma \kappa \rho^{\gamma-1} & 2 m / \rho\end{array}\right)$.

Many properties of the system are derived from the resolution of the Riemann problem, that is, the Cauchy problem when the initial data has the shape of a step-function:

$$
U(0, x)=\left(\rho_{l}, m_{l}\right) \quad \text { for } x<0 \quad \text { and } \quad U(0, x)=\left(\rho_{r}, m_{r}\right) \quad \text { for } x>0,
$$

where $\left(\rho_{l}, m_{l}\right)$ and $\left(\rho_{r}, m_{r}\right)$ are two fixed states in $\mathbb{R}^{+*} \times \mathbb{R}$. The resolution of this problem leads to the introduction of the following classical objects which we list below.

We denote by $\hat{r}_{i}(u)$ the following right eigenvectors of $A(u)$ corresponding to its $i$-th eigenvalue:

$$
\hat{r}_{1}=\left(\begin{array}{l}
1 \\
\lambda_{1}
\end{array}\right) \quad \text { and } \quad \hat{r}_{2}=\left(\begin{array}{l}
1 \\
\lambda_{2}
\end{array}\right) .
$$

Note that $\nabla \lambda_{i}(u) \cdot \hat{r_{i}}(u) \neq 0$ for any $u \in \mathbb{R}^{+*} \times \mathbb{R}$, that is, the two fields are genuinely nonlinear in the sense of Lax (see [25]). We can renormalize these eigenvectors in order to get $\nabla \lambda_{i}(u) \cdot r_{i}(u)=1$ :

$$
r_{1}=\frac{-2 \rho^{(3-\gamma) / 2}}{\sqrt{\kappa \gamma}(\gamma+1)}\left(\begin{array}{l}
1 \\
\lambda_{1}
\end{array}\right) \quad \text { and } \quad r_{2}=\frac{2 \rho^{(3-\gamma) / 2}}{\sqrt{\kappa \gamma}(\gamma+1)}\left(\begin{array}{l}
1 \\
\lambda_{2}
\end{array}\right) .
$$

We introduce the pair (6) of Riemann invariants of the system, which is a couple of functions $\left(w_{1}, w_{2}\right)$ satisfying $\nabla w_{i} \cdot r_{i}=0$, which here provide other coordinates for the state of the system. We will consider them as variables and as functions $u \mapsto w^{i}(u)$ as well.

\subsection{Wave curves}

Rarefaction and shock curves. Rarefaction curves are made of states $u_{r}$ (on the right) that can be connected to a state $u_{l}$ (on the left) by rarefaction waves, that is, smooth selfsimilar solutions of the Riemann problem, associated with either eigenvalue $\lambda_{1}$ and $\lambda_{2}$. We denote these curves by $R_{i}$. Here they are given by the following equations:

1-rarefactions: $m_{r}-m_{l}=\frac{m_{l}}{\rho_{l}}\left(\rho_{r}-\rho_{l}\right)-\frac{2 \sqrt{\kappa \gamma}}{\gamma-1} \rho_{r}\left(\rho_{r}^{(\gamma-1) / 2}-\rho_{l}^{(\gamma-1) / 2}\right)$ with $\rho_{r}<\rho_{l}$,

2-rarefactions: $m_{r}-m_{l}=\frac{m_{l}}{\rho_{l}}\left(\rho_{r}-\rho_{l}\right)+\frac{2 \sqrt{\kappa \gamma}}{\gamma-1} \rho_{r}\left(\rho_{r}^{(\gamma-1) / 2}-\rho_{l}^{(\gamma-1) / 2}\right)$ with $\rho_{r}>\rho_{l}$. 
Shock curves of the $i$-th family are made of states $u_{r}$ (on the right) that can be connected to a state $u_{l}$ (on the left) by an $i$-shock, that is, a discontinuity that propagates at constant speed $s$, satisfying the Rankine-Hugoniot relation

$$
[f(u)]=s[u]
$$

(where $[\phi]$ denotes the difference $\phi_{r}-\phi_{l}$ across the discontinuity, and $s$ the speed of the shock), and the admissibility conditions of Lax:

$$
\begin{aligned}
& s<\lambda_{1}\left(u_{l}\right) \quad \text { and } \quad \lambda_{1}\left(u_{r}\right)<s<\lambda_{2}\left(u_{r}\right) \quad \text { for a shock of the first family, } \\
& s>\lambda_{2}\left(u_{r}\right) \quad \text { and } \quad \lambda_{1}\left(u_{l}\right)<s<\lambda_{2}\left(u_{l}\right) \quad \text { for a shock of the second family. }
\end{aligned}
$$

After computation, these curves are given by:

$$
\begin{aligned}
& \text { 1-shocks: } m_{r}=m_{l}+\frac{m_{l}}{\rho_{l}}\left(\rho_{r}-\rho_{l}\right)-\sqrt{\kappa \frac{\rho_{r}}{\rho_{l}} \frac{\rho_{r}^{\gamma}-\rho_{l}^{\gamma}}{\rho_{r}-\rho_{l}}}\left(\rho_{r}-\rho_{l}\right) \quad\left(=: S_{1}\left(\rho_{r}, u_{l}\right)\right) \\
& \text { with the condition } \rho_{r}>\rho_{l}, \quad(30) \\
& \text { 2-shocks: } m_{r}=m_{l}+\frac{m_{l}}{\rho_{l}}\left(\rho_{r}-\rho_{l}\right)+\sqrt{\kappa \frac{\rho_{r}}{\rho_{l}} \frac{\rho_{r}^{\gamma}-\rho_{l}^{\gamma}}{\rho_{r}-\rho_{l}}}\left(\rho_{r}-\rho_{l}\right) \quad\left(=: S_{2}\left(\rho_{r}, u_{l}\right)\right) \\
& \quad \text { with the condition } \rho_{r}<\rho_{l} .
\end{aligned}
$$

Let us agree to extend the definition of the shock speed $s\left(u_{1}, u_{2}\right)$ to states that do not satisfy the Rankine-Hugoniot relations (but are such that $\rho_{1} \neq \rho_{2}$ ) by the following expression:

$$
s\left(u_{1}, u_{2}\right):=\frac{m_{2}-m_{1}}{\rho_{2}-\rho_{1}} .
$$

It should be noted that, by Taylor's formula, on the $i$-th rarefaction curves, the "shock speed" admits the following expansion: for $u_{r}=R_{i}\left(s, u_{l}\right)$,

$$
s\left(u_{l}, u_{r}\right)=\frac{\lambda_{i}\left(u_{l}\right)+\lambda_{i}\left(u_{r}\right)}{2}+O\left(\left|u_{l}-u_{r}\right|^{2}\right) .
$$

Wave curves. Merging shocks and rarefaction curves allows us to introduce Lax's wave curves $\tilde{\Phi}_{1}$ and $\tilde{\Phi}_{2}$ :

$$
\tilde{\Phi}_{1}\left(\rho, u_{l}\right)= \begin{cases}\left(\rho, m_{l}+\frac{m_{l}}{\rho_{l}}\left(\rho-\rho_{l}\right)-\frac{2 \sqrt{\kappa \gamma}}{\gamma-1} \rho\left(\rho^{(\gamma-1) / 2}-\rho_{l}^{(\gamma-1) / 2}\right)\right) & \text { for } \rho \leq \rho_{l}, \\ \left(\rho, \frac{m_{l}}{\rho_{l}} \rho-\sqrt{\kappa \frac{\rho}{\rho_{l}}\left(\rho^{\gamma}-\rho_{l}^{\gamma}\right)\left(\rho-\rho_{l}\right)}\right) & \text { for } \rho \geq \rho_{l},\end{cases}
$$




$$
\tilde{\Phi}_{2}\left(\rho, u_{l}\right)= \begin{cases}\left(\rho, \frac{m_{l}}{\rho_{l}} \rho-\sqrt{\kappa \frac{\rho}{\rho_{l}}\left(\rho^{\gamma}-\rho_{l}^{\gamma}\right)\left(\rho-\rho_{l}\right)}\right) & \text { for } \rho \leq \rho_{l}, \\ \left(\rho, m_{l}+\frac{m_{l}}{\rho_{l}}\left(\rho-\rho_{l}\right)+\frac{2 \sqrt{\kappa \gamma}}{\gamma-1} \rho\left(\rho^{(\gamma-1) / 2}-\rho_{l}^{(\gamma-1) / 2}\right)\right. & \text { for } \rho \geq \rho_{l} .\end{cases}
$$

One may parameterize these curves with a coordinate different from $\rho$ : it is a consequence of $\nabla \lambda_{i} \cdot r_{i}=1$ that

$$
\sigma_{i}:=\lambda_{i}\left(u_{r}\right)-\lambda_{i}\left(u_{l}\right)
$$

increases with $u_{r}$ along the rarefaction curve (that is, as $\rho_{r} \leq \rho_{l}$ decreases for a 1rarefaction, as $\rho_{r} \geq \rho_{l}$ increases for a 2-rarefaction). On the other hand, one sees that

$$
\sigma_{i}:=2\left(\lambda_{i}\left(u_{r}\right)-s\right),
$$

where $s$ is the speed of the shock, decreases along the shock curve (that is, as $\rho_{r} \geq \rho_{l}$ increases for a 1-shock, as $\rho_{r} \leq \rho_{l}$ decreases for a 2-shock). Moreover, the resulting parameterization is twice continuously differentiable (see [25]). We denote by $\Phi_{i}$ the wave curves with the above described parameterization.

Curves in Riemann coordinates. Another parameterization for these curves is obtained by using Riemann coordinates: this provides two curves $w^{2} \mapsto \hat{\Phi}_{1}\left(w^{2}, u\right)$ and $w^{1} \mapsto$ $\hat{\Phi}_{2}\left(w^{1}, u\right)$ where the states are considered in Riemann coordinates. It is elementary to deduce from $(6)$ and $(26)-(27)$ that along the curve $R_{i}, w^{i}$ is constant, while $w^{3-i}$ increases. On the other hand, it follows from (6), (30)- 31 , and the Cauchy-Schwarz inequality that along the curve $S_{i}$, both Riemann invariants decrease.

We will denote by $\hat{S}_{1}\left(w^{2}, u_{l}\right)$ and $\hat{S}_{2}\left(w^{1}, u_{l}\right)$ the Rankine-Hugoniot curves in Riemann coordinates (not necessarily satisfying 29$)$ ); that is, $\hat{S}_{1}\left(w^{2}, u_{l}\right)$ is the point on the first Hugoniot locus starting at $u_{l}$, having $w^{2}$ as second Riemann invariant.

Approximations of the axes via wave curves. We finish this subsection by introducing the following "approximations of the axes", in Riemann coordinates, obtained by gluing some pieces of wave curves. Given a base point $u$, the curves $\Psi_{i}^{k}$ are defined for $i=1,2$ and $k \in \mathbb{N}$ as follows:

- For $n=0$ the curves $\Psi_{1}^{0}$ and $\Psi_{2}^{0}$ are defined to be the curves $\hat{\Phi}_{i}$.

- For $n \geq 1$, we define $\Psi_{1}^{n}: w^{2} \mapsto \Psi_{1}^{n}\left(w^{2}, u\right)$ and $\Psi_{2}^{n}: w^{1} \mapsto \Psi_{1}^{n}\left(w^{1}, u\right)$ as follows:

- 1-curves: for $w^{2} \geq w^{2}(u), \Psi_{1}^{n}$ is defined to be the rarefaction curve, that is, the axis $w^{1}=w^{1}(u)$. For $w^{2} \leq w^{2}(u)$ we define the points $\mathfrak{u}_{m}$ recursively by $\mathfrak{u}_{0}=u$ and $\mathfrak{u}_{m+1}=\hat{S}_{1}\left(w^{2}\left(\mathfrak{u}_{m}\right)-1 / n, \mathfrak{u}_{m}\right)$ for any $m \in \mathbb{N}$. Then the curve $w^{2} \mapsto \Psi_{1}^{n}\left(w^{2}, u\right)$ is defined by $\Psi_{1}^{n}\left(w^{2}, u\right)=\hat{S}_{1}\left(w^{2}, \mathfrak{u}_{m}\right)$ for $w^{2}\left(\mathfrak{u}_{m+1}\right) \leq w^{2} \leq w^{2}\left(\mathfrak{u}_{m}\right)$.

- 2-curves: for $w^{1} \geq w^{1}(u), \Psi_{2}^{n}$ is defined to be the rarefaction curve, that is, the axis $w^{2}=w^{2}(u)$. For $w^{1} \leq w^{1}(u)$ we define the points $\mathfrak{u}_{m}$ recursively by $\mathfrak{u}_{0}=u$ and $\mathfrak{u}_{m+1}=\hat{S}_{2}\left(w^{1}\left(\mathfrak{u}_{m}\right)-1 / n, \mathfrak{u}_{m}\right)$ for any $m \in \mathbb{N}$, as long as the point does not meet the vacuum. Then the curve $w^{1} \mapsto \Psi_{1}^{n}\left(w^{1}, u\right)$ is defined by $\Psi_{2}^{n}\left(w^{1}, u\right)=\hat{S}_{2}\left(w^{1}, \mathfrak{u}_{m}\right)$ for $w^{1}\left(\mathfrak{u}_{m+1}\right) \leq w^{1} \leq w^{1}\left(\mathfrak{u}_{m}\right)$.

The curves $\Psi_{1}^{n}$ and $\Psi_{2}^{n}$ are illustrated in Figure 2 


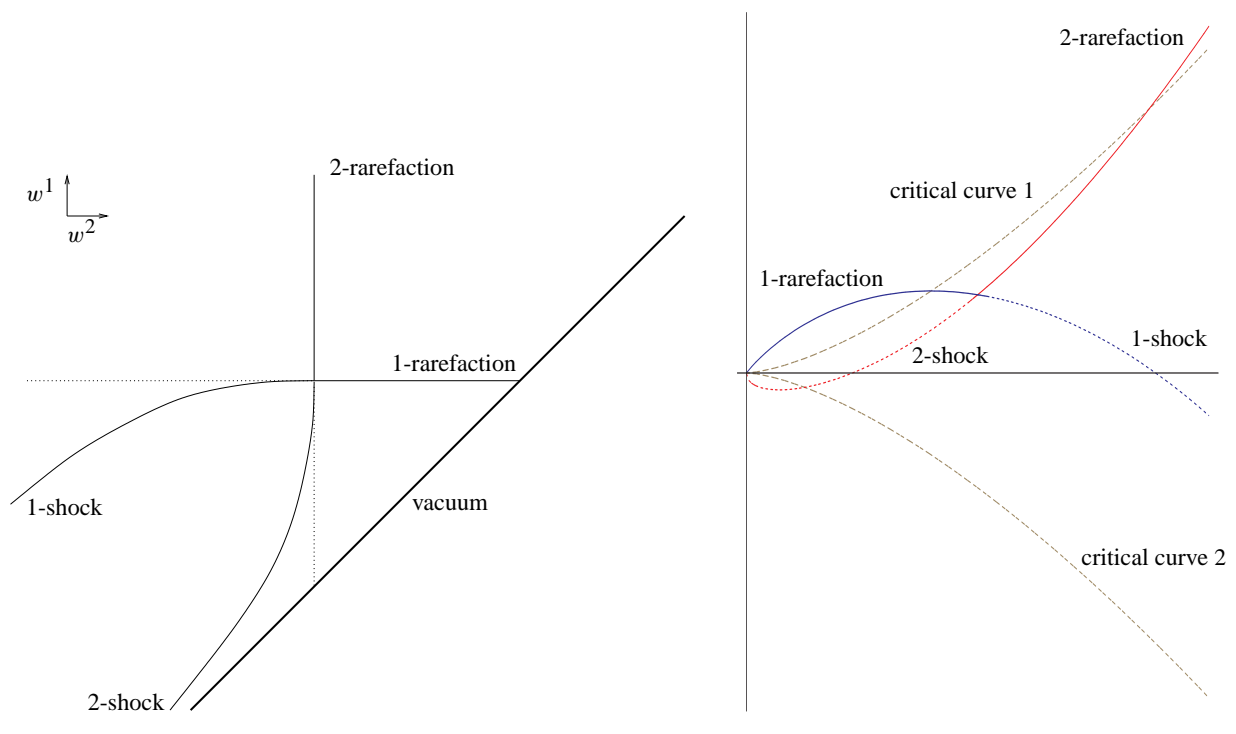

(a) $\left(w^{1}, w^{2}\right)$ coordinates

(b) $(\rho, m)$ coordinates

Fig. 1. Wave curves.

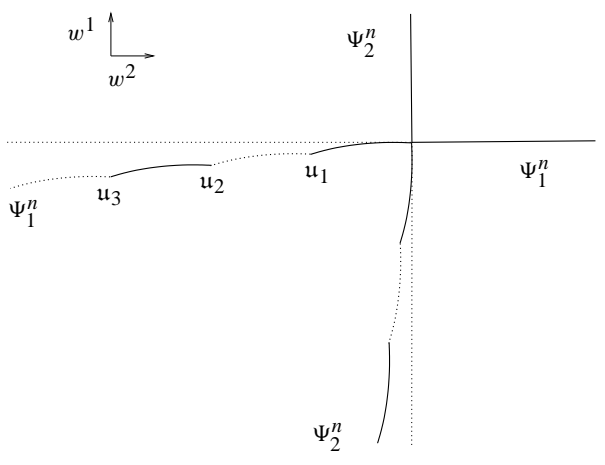

Fig. 2. The curves $\Psi_{1}^{n}$ and $\Psi_{2}^{n}$.

As the Rankine-Hugoniot curve and the rarefaction curve are smooth and have a $C^{2}$ contact at the base point (see [25]), it follows that

$$
\left|\hat{S}_{i}\left(w^{3-i}, u\right)-\hat{\Phi}_{i}\left(w^{3-i}, u\right)\right|=O\left(\left|w^{3-i}-w^{3-i}(u)\right|^{3}\right) .
$$

Hence, for each compact $K \subset \mathbb{R}^{+*} \times \mathbb{R}$, there is a constant $C_{K}>0$ such that

$$
\begin{aligned}
& \left|\Psi_{1}^{n}\left(w^{2}, u\right)-\left(w^{1}(u), w^{2}\right)\right| \leq \frac{C_{K}\left|w_{2}-w_{2}(u)\right|}{n^{2}}, \\
& \left|\Psi_{2}^{n}\left(w^{1}, u\right)-\left(w^{1}, w^{2}(u)\right)\right| \leq \frac{C_{K}\left|w_{1}-w_{1}(u)\right|}{n^{2}},
\end{aligned}
$$


as long as the states considered are in $K$. (Note that $\Psi_{1}^{n}$ is below the $w^{2}$-axis and $\Psi_{2}^{n}$ is to the left of the $w^{1}$-axis, because the corresponding shock curves are.)

Generalities. Finally, we add $l$ as an exponent to denote left curves, i.e. the sets of points that are connected as left states to a given right state by either a shock or a rarefaction wave.

The classical theorem of Lax proves that, for general hyperbolic systems of conservation laws with characteristic fields which are either genuinely nonlinear or linearly degenerate, one can solve the Riemann problem between two states sufficiently close to each other, in terms of (small) waves which are either shocks, rarefaction waves or contact discontinuities. Concerning the system $\overline{E I}$, the Riemann problem can be solved globally, but vacuum may appear between two rarefaction waves: we refer for instance to [34].

\subsection{Notations}

We will always put the time variable $t$ before the space variable $x$. Hence we make the convention that in the product $[a, b] \times[c, d]$ the time variable $t$ describes $[a, b]$ whereas the space variable $x$ describes $[c, d]$.

We introduce several regions in $\mathbb{R}^{+*} \times \mathbb{R}$ :

$$
\begin{aligned}
& \mathcal{D}_{1}=\left\{(\rho, m) \in \mathbb{R}^{+*} \times \mathbb{R}: \lambda_{1}(\rho, m)>0\right\}, \\
& \mathcal{D}_{2}=\left\{(\rho, m) \in \mathbb{R}^{+*} \times \mathbb{R}: \lambda_{1}(\rho, m)<0<\lambda_{2}(\rho, m)\right\}, \\
& \mathcal{D}_{3}=\left\{(\rho, m) \in \mathbb{R}^{+*} \times \mathbb{R}: \lambda_{2}(\rho, m)<0\right\}, \\
& \mathcal{C}_{1}=\left\{(\rho, m) \in \mathbb{R}^{+*} \times \mathbb{R}: \lambda_{1}(\rho, m)=0\right\}, \\
& \mathcal{C}_{2}=\left\{(\rho, m) \in \mathbb{R}^{+*} \times \mathbb{R}: \lambda_{2}(\rho, m)=0\right\} .
\end{aligned}
$$

We refer to $\mathcal{C}_{1}$ and $\mathcal{C}_{2}$ as critical curves. Of course one has

$$
\mathbb{R}^{+*} \times \mathbb{R}=\mathcal{D}_{1} \amalg \mathcal{D}_{2} \amalg \mathcal{D}_{3} \amalg \mathcal{C}_{1} \amalg \mathcal{C}_{2} .
$$

Finally, given a simple wave $\left(u_{-}, u_{+}\right)$with $u_{+}=\Phi_{i}\left(\sigma, u_{-}\right)$, we will call $\sigma$ the wave amplitude and $|\sigma|$ the strength of the wave. When the wave is called $\alpha$, we denote its amplitude by $\sigma_{\alpha}$. When specified, we may use as a strength of the wave the value $\left|w^{3-i}-w^{3-i}(u-)\right|$ if $u_{+}=\hat{\Phi}_{i}\left(w^{3-i}, u_{-}\right)$. A couple $\left(\sigma_{1}, \sigma_{2}\right)$ describing a Riemann problem is called a wave-vector. For simple waves, we may identify the amplitude and the wave-vector.

\subsection{Main ideas of the proof}

The proof is divided into three steps, which we develop in separate sections:

- In Section 3, we show how to steer any initial state $u_{0}:=\left(\rho_{0}, m_{0}\right)$ in $B V([0,1]$; $\mathbb{R}^{+*} \times \mathbb{R}$ ) with small variation and with $\rho_{0} \geq \underline{\rho}_{0}>0$ to a constant state (not necessarily given in advance). 
- In Section 4, it is shown how one can drive the system from a given constant state $\omega_{0}$ to any other constant state $\omega_{1}$.

- Finally, in Section 5 it is proven how a state $u_{1}=\left(\rho_{1}, m_{1}\right)$ as in Theorem 1 can be attained, starting from a certain constant state (which depends on $u_{1}$ ).

The principal idea in Section 3 is to destabilize the system by letting a strong shock enter the domain. As mentioned in Subsection 1.4, one can doubt that boundary conditions with small total variation are enough to get rid of the structure of the initial condition. With a strong shock entering the domain (for instance from the left, that is, through the boundary $x=0$ ), one can hope that all the information contained in the initial condition can be shifted outside the domain. This is an application of the "return method" introduced by J.- M. Coron in [14] in the context of finite-dimensional systems, and used since for various PDE problems such as Euler incompressible equation [15, 19], Burgers equation [24], Vlasov-Poisson equation [20], Schrödinger equation [6, 7]. Note that the existence of a solution of the system with initial condition given by a small $B V$ perturbation of a strong shock has been studied by several authors (see in particular [32], [10], [13], [27] and [31]; see also [1]).

The second step of the control process, developed in Section 4 is quite elementary: one drives a constant state to another simply by solving several Riemann problems one after another, that is, the two states are separated by several shocks and centered rarefaction waves (passing either through the left or the right side of the domain according to the sign of their speed).

The last step is done by a (backward) front-tracking algorithm. We construct, backward in time, a sequence of piecewise constant functions $U_{n} \in L^{\infty}([-T, 0] ; B V([0,1]$; $\mathbb{R}^{+*} \times \mathbb{R}$ )) (for an appropriate $T>0$ ) which converges to a solution of the system with $u_{\mid t=0}=u_{1}$ and $u_{\mid t=-T}$ equal to a constant state. The different constant states are separated either by shocks or by (small) rarefaction fronts (as in the classical front-tracking algorithm, see [8]). We start from an approximation of $u_{1}$ at time $t=0$, and then solve (approximately) "backward Riemann problems". In fact, we add to $u_{1}$ one or two (strong) shocks which should enter the domain (for $t<0$ ). When interaction occurs, we solve it as in the usual front-tracking algorithm, except when a rarefaction front meets one of the two strong shocks, in which case the problem is solved in terms of two shocks of the same family. The main issue here is to avoid the situation where the rarefaction fronts meet and merge (which would result in a non-entropic solution). We manage to make these rarefaction fronts either quit the domain or meet one of the strong shocks before any possible encounter with another rarefaction front. This justifies the Oleı̆nik-type constraints that we impose on $u_{1}$ in Theorem 1

\section{Step 1: getting rid of the initial condition}

The goal of this section is to prove the following proposition:

Proposition 1. Let $u_{0} \in B V\left([0,1] ; \mathbb{R}^{+*} \times \mathbb{R}\right)$ be as in Theorem 1 Then there exist $T_{1}>0$, a constant state $\omega_{1} \in \mathbb{R}^{+*} \times \mathbb{R}$, and an entropy solution $u:\left[0, T_{1}\right] \times[0,1] \rightarrow$ 
$\mathbb{R}^{+*} \times \mathbb{R}$ of $(\mathrm{EI})$ such that

$$
\begin{gathered}
u_{\mid t=0}=u_{0}, \\
u_{\mid t=T_{1}}=\omega_{1} .
\end{gathered}
$$

Before getting to the proof, we need some preliminary material needed to handle strong shocks, which as we explained earlier are the main tool in this part.

\subsection{Preliminaries}

Here we recall some results on the solvability of the Riemann problem in the neighborhood of a strong shock, and on the interaction of a small wave with a strong shock.

These results are derived under the following stability condition on the strong shock (due to Majda, see [30]): a shock (of the $j$-th family) $\left(\tilde{u}^{-}, \tilde{u}^{+}\right.$) with speed $\tilde{s}$ and wave amplitude $\tilde{\varepsilon}$ is said to be Majda-stable if:

(i) $\tilde{s}$ is not an eigenvalue of $\frac{\partial f}{\partial u}\left(\tilde{u}^{ \pm}\right)$,

(ii) $\left\{r_{j}\left(\tilde{u}^{+}\right): \lambda_{j}\left(\tilde{u}^{+}\right)>s\right\} \cup\left\{\tilde{u}^{+}-\tilde{u}^{-}\right\} \cup\left\{r_{j}\left(\tilde{u}^{-}\right): \lambda_{j}\left(\tilde{u}^{-}\right)<s\right\}$ is a basis of $\mathbb{R}^{n}$.

The second condition for a 1 -shock (resp. a 2 -shock) reduces here to: $\left\{\tilde{u}^{+}-\tilde{u}^{-}, r_{2}\left(\tilde{u}^{+}\right)\right\}$ (resp. $\left\{r_{1}\left(\tilde{u}^{-}\right), \tilde{u}^{+}-\tilde{u}^{-}\right\}$) is a basis of $\mathbb{R}^{2}$. The condition 41$\}$ is stronger than Lax entropy inequalities, and is satisfied by any shock for (EI).

We have the following result (see [32, Lemma 3.1], and also [13, 31]):

Lemma 1. Suppose that the shock $\left(\tilde{u}^{-}, \tilde{u}^{+}\right)$with wave-vector $\tilde{\varepsilon}$ is Majda stable. Then the Riemann problems $\left(u^{-}, u^{+}\right)$close to $\left(\tilde{u}^{-}, \tilde{u}^{+}\right)$have a unique solution with wave-vector $\varepsilon$ close to $\tilde{\varepsilon}$. Moreover, if $\gamma$ is the wave-vector of a small wave interacting with $\left(\tilde{u}^{-}, \tilde{u}^{+}\right)$, then, up to higher order terms in $|\gamma|$, the resulting wave is given by $\tilde{\varepsilon}+\varepsilon^{\prime}$, with $\varepsilon^{\prime}$ given by:

- Case of a strong 2-shock wave

- Small wave interacting from the right side:

$$
\begin{aligned}
\left(\begin{array}{cc}
\left(\lambda_{1}\left(\tilde{u}_{-}\right)-\tilde{s}\right) l_{1}\left(\tilde{u}_{+}\right) \cdot r_{1}\left(\tilde{u}_{-}\right) & \frac{\partial s}{\partial \varepsilon^{-}}\left(\tilde{u}_{-}\right) l_{1}\left(\tilde{u}_{+}\right) \cdot\left[\tilde{u}_{+}-\tilde{u}_{-}\right] \\
\left(\lambda_{2}\left(\tilde{u}_{-}\right)-\tilde{s}\right) l_{2}\left(\tilde{u}_{+}\right) \cdot r_{1}\left(\tilde{u}_{-}\right) & \frac{\partial s}{\partial \varepsilon^{-}}\left(\tilde{u}_{-}\right) l_{2}\left(\tilde{u}_{+}\right) \cdot\left[\tilde{u}_{+}-\tilde{u}_{-}\right]
\end{array}\right)\left(\begin{array}{c}
\varepsilon_{1}^{\prime} \\
\varepsilon_{2}^{\prime}
\end{array}\right) \\
=\left(\begin{array}{cc}
\lambda_{1}\left(\tilde{u}_{+}\right)-\tilde{s} & 0 \\
0 & \lambda_{2}\left(\tilde{u}_{+}\right)-\tilde{s}
\end{array}\right)\left(\begin{array}{l}
\gamma_{1} \\
\gamma_{2}
\end{array}\right) .
\end{aligned}
$$

- Small wave interacting from the left side:

$$
\begin{aligned}
\left(\begin{array}{cc}
\frac{\partial s}{\partial \varepsilon^{+}}\left(\tilde{u}_{+}\right) l_{2}\left(\tilde{u}_{+}\right) \cdot\left[\tilde{u}_{+}-\tilde{u}_{-}\right] & 0 \\
\frac{\partial s}{\partial \varepsilon^{+}}\left(\tilde{u}_{+}\right) l_{1}\left(\tilde{u}_{+}\right) \cdot\left[\tilde{u}_{+}-\tilde{u}_{-}\right] & \left(\lambda_{1}\left(\tilde{u}_{-}\right)-\tilde{s}\right)
\end{array}\right) & \left(\begin{array}{l}
\varepsilon_{1}^{\prime} \\
\varepsilon_{2}^{\prime}
\end{array}\right) \\
= & \left(\begin{array}{cc}
\lambda_{2}\left(\tilde{u}_{-}\right)-\tilde{s} & 0 \\
0 & 0
\end{array}\right)\left(\begin{array}{c}
\gamma_{2} \\
0
\end{array}\right) .
\end{aligned}
$$


- Case of a strong 1-shock wave

- Small wave interacting from the right side:

$$
\begin{aligned}
\left(\begin{array}{cc}
\frac{\partial s}{\partial \varepsilon^{-}}\left(\tilde{u}_{-}\right) l_{1}\left(\tilde{u}_{+}\right) \cdot\left[\tilde{u}_{+}-\tilde{u}_{-}\right] & 0 \\
\frac{\partial s}{\partial \varepsilon^{-}}\left(\tilde{u}_{-}\right) l_{2}\left(\tilde{u}_{+}\right) \cdot\left[\tilde{u}_{+}-\tilde{u}_{-}\right] & \left(\lambda_{2}\left(\tilde{u}_{-}\right)-\tilde{s}\right)
\end{array}\right)\left(\begin{array}{c}
\varepsilon_{1}^{\prime} \\
\varepsilon_{2}^{\prime}
\end{array}\right) & \\
& =\left(\begin{array}{cc}
\lambda_{1}\left(\tilde{u}_{+}\right)-\tilde{s} & 0 \\
0 & 0
\end{array}\right)\left(\begin{array}{c}
\gamma_{1} \\
0
\end{array}\right)
\end{aligned}
$$

- Small wave interacting from the left side:

$$
\begin{array}{r}
\left(\begin{array}{cc}
\frac{\partial s}{\partial \varepsilon_{+}}\left(\tilde{u}_{+}\right) l_{1}\left(\tilde{u}_{-}\right) \cdot\left[\tilde{u}_{+}-\tilde{u}_{-}\right] & \left(\lambda_{2}\left(\tilde{u}_{+}\right)-\tilde{s}\right) l_{1}\left(\tilde{u}_{-}\right) \cdot r_{2}\left(\tilde{u}_{+}\right)-\left(r_{2}\left(\tilde{u}_{+}\right) \cdot \nabla_{u} s\left(\tilde{\varepsilon}_{+}, \tilde{u}_{+}\right)\right) l_{1}\left(\tilde{u}_{-}\right) \cdot\left[\tilde{u}_{+}-\tilde{u}_{-}\right] \\
\frac{\partial s}{\partial \varepsilon^{+}}\left(\tilde{u}_{+}\right) l_{2}\left(\tilde{u}_{-}\right) \cdot\left[\tilde{u}_{+}-\tilde{u}_{-}\right] & \left(\lambda_{2}\left(\tilde{u}_{+}\right)-\tilde{s}\right) l_{2}\left(\tilde{u}_{-}\right) \cdot r_{2}\left(\tilde{u}_{+}\right)-\left(r_{2}\left(\tilde{u}_{+}\right) \cdot \nabla_{u} s\left(\tilde{\varepsilon}_{+}, \tilde{u}_{+}\right)\right) l_{2}\left(\tilde{u}_{-}\right) \cdot\left[\tilde{u}_{+}-\tilde{u}_{-}\right]
\end{array}\right) \\
\cdot\left(\begin{array}{c}
\varepsilon_{1}^{\prime} \\
\varepsilon_{2}^{\prime}
\end{array}\right)=\left(\begin{array}{cc}
\lambda_{1}\left(\tilde{u}_{+}\right)-\tilde{s} & 0 \\
0 & \lambda_{2}\left(\tilde{u}_{+}\right)-\tilde{s}
\end{array}\right)\left(\begin{array}{l}
\gamma_{1} \\
\gamma_{2}
\end{array}\right) .
\end{array}
$$

We will use Lemma 1 by means of the following corollary (see also [1, Lemma A3], [13], [31, Lemmas 3.3-3.6]). We describe the situation when the family of the strong shock $k$ is equal to 2 , but of course a similar result stands for $k=1$.

Corollary 1. Let $\left(\tilde{u}^{-}, \tilde{u}^{+}\right)$be a strong 2-shock for system $(\mathrm{EI}): \tilde{u}^{+}=\Phi_{2}\left(\tilde{\varepsilon}_{2}, \tilde{u}^{-}\right)$. Fix $\tilde{\varepsilon}_{1}=0$. Then there exist neighborhoods $\mathcal{V}_{1}^{-}, \mathcal{V}_{1}^{+}$and $\tilde{\mathcal{E}}$ respectively of $\tilde{u}^{-}$and $\tilde{u}^{+}$in $\mathbb{R}^{+*} \times \mathbb{R}$ and of $\tilde{\varepsilon}_{2}$ in $\mathbb{R}^{-*}$ such that for all $\left(u^{-}, u^{+}\right) \in \mathcal{V}_{1}^{-} \times \mathcal{V}_{1}^{+}$, the Riemann problem $\left(u^{-}, u^{+}\right)$is (uniquely) solvable, that is,

$$
u^{+}=\Phi_{2}\left(\varepsilon_{2}, \Phi_{1}\left(\varepsilon_{1}, u^{-}\right)\right) \quad \text { with } \quad\left|\varepsilon_{j}-\tilde{\varepsilon}_{j}\right|=O(1) \max \left(\left|u^{+}-\tilde{u}^{+}\right|,\left|u^{-}-\tilde{u}^{-}\right|\right) .
$$

Moreover, there is a constant $V$ depending only on $\mathcal{V}_{1}^{-}$and $\mathcal{V}_{1}^{+}$such that, $\left(u^{-}, u^{+}\right) \in$ $\mathcal{V}_{1}^{-} \times \mathcal{V}_{1}^{+}$being a strong shock, say $u^{+}=\Phi_{2}\left(\varepsilon_{2}, u^{-}\right), \varepsilon_{2} \in \tilde{\mathcal{E}}$ :

(i) (Interaction on the left of the strong shock) Consider a small wave $\gamma\left(u^{--}, u^{-}\right)$, say $u^{-}=\Phi_{j}\left(\alpha, u^{--}\right)$. Then its interaction with the strong shock is described by

$$
u^{+}=\Phi_{2}\left(\varepsilon_{2}\left(\Phi_{1}\left(\varepsilon_{1}, u^{--}\right)\right)\right) \quad \text { with } \quad\left|\varepsilon_{2}-\tilde{\varepsilon}_{2}\right|+\left|\varepsilon_{1}\right| \leq V|\alpha| .
$$

(ii) (Interaction on the right of the strong shock) Consider a small wave $\gamma\left(u^{+}, u^{++}\right)$, say $u^{++}=\Phi_{j}\left(\alpha, u^{+}\right)$. Then its interaction with the strong shock is described by

$$
u^{++}=\Phi_{2}\left(\varepsilon_{2}\left(\Phi_{1}\left(\varepsilon_{1}, u^{-}\right)\right)\right) \text {with } \quad\left|\varepsilon_{2}-\tilde{\varepsilon}_{2}\right|+\left|\varepsilon_{1}\right| \leq V|\alpha|
$$




\subsection{Starting point in the proof of Proposition 1}

The strategy of the proof of Proposition 1 is the following: find a strong 2-shock on the left such that the initial-value problem on $\mathbb{R}$ with as initial state the function composed of: the left state of this strong shock on the left of the domain, $u_{0}$ inside $[0,1]$, and a constant value not far from $u_{0}$ on the right of the domain, admits a well-defined solution on $\mathbb{R}$, which reaches a constant state inside $[0,1]$ in finite time. We begin with a lemma.

Lemma 2. For any $\omega \in \mathbb{R}^{+*} \times \mathbb{R}$, there exist $\sigma<0$ and $\omega^{\prime} \in \mathbb{R}^{+*} \times \mathbb{R}$ such that $\omega=\Phi_{2}\left(\sigma, \omega^{\prime}\right)$ and

$$
\begin{gathered}
\lambda_{2}\left(\omega^{\prime}\right)>\lambda_{1}\left(\omega^{\prime}\right) \geq 3, \\
s\left(\omega, \omega^{\prime}\right) \geq 3 .
\end{gathered}
$$

Proof. Define $\omega=:\left(\rho_{r}, m_{r}\right)$. One considers the curve of 2-shocks on the left

$$
\left[\rho_{r},+\infty\right) \ni \rho_{l} \mapsto\left(\rho_{l}, m_{l}\right) \quad \text { with } \quad \frac{m_{l}}{\rho_{l}}=\frac{m_{r}}{\rho_{r}}+\sqrt{\kappa \frac{1}{\rho_{l} \rho_{r}} \frac{\rho_{l}^{\gamma}-\rho_{r}^{\gamma}}{\rho_{l}-\rho_{r}}}\left(\rho_{l}-\rho_{r}\right) .
$$

Comparing the growth of the second term on the right hand side with the growth of $\rho_{l}^{(\gamma-1) / 2}$, one easily sees that $\lambda_{1}\left(\rho_{l}, m_{l}\right) \geq 3$ for $\rho_{l}$ large enough. Using the RankineHugoniot equation one gets

$$
s=\frac{m_{r}}{\rho_{r}}+\sqrt{\kappa \frac{\rho_{l}}{\rho_{r}} \frac{\rho_{l}^{\gamma}-\rho_{r}^{\gamma}}{\rho_{l}-\rho_{r}}},
$$

which establishes (46) for $\rho_{l}$ large as well.

Now by Lemma 2 applied to the constant state $\bar{u}_{0}$, there exists $\bar{v}_{0} \in \mathbb{R}^{+*} \times \mathbb{R}$ such that $\bar{u}_{0}=\Phi_{2}\left(\sigma_{0}, \bar{v}_{0}\right)$ for some $\sigma_{0}<0$ and $\lambda_{2}\left(\bar{v}_{0}\right)>\lambda_{1}\left(\bar{v}_{0}\right) \geq 3$. We introduce the following function $U_{0} \in B V_{\text {loc }}\left(\mathbb{R} ; \mathbb{R}^{+*} \times \mathbb{R}\right)$ :

$$
U_{0}(x)= \begin{cases}\bar{v}_{0} & \text { for } x<0 \\ u_{0}(x) & \text { for } 0 \leq x \leq 1 \\ \bar{u}_{0} & \text { for } x>1\end{cases}
$$

With $U_{0}$ defined in this way, Proposition 1 is a direct consequence of the following one:

Proposition 2. If $u_{0}$ has small enough total variation, then there is a global-in-time entropic solution $U$ of $(\mathbb{E I})$ in $[0,+\infty) \times \mathbb{R}$ satisfying

$$
U(0, \cdot)=U_{0} \quad \text { in } \mathbb{R} .
$$

Moreover,

$$
U_{\mid\{1\} \times[0,1]} \text { is constant. }
$$


One deduces Proposition 1 simply by taking the restriction of $U$ to $[0,1] \times[0,1]$. The rest of this section is devoted to the proof of Proposition 2 by a front-tracking algorithm. The approximations that we construct are intended to take values in the domain

$$
\mathfrak{D}:=B\left(\bar{v}_{0} ; r\right) \cup B\left(\bar{u}_{0} ; r\right),
$$

with $r$ so small that:

- the characteristic speeds are uniformly strictly separated in $B\left(\bar{v}_{0} ; r\right)$ and in $B\left(\bar{u}_{0} ; r\right)$,

- any interactions inside $B\left(\bar{v}_{0} ; r\right)$ or inside $B\left(\bar{u}_{0} ; r\right)$ are well-defined (without vacuum) with Glimm's estimates valid,

- any simple wave joining a state in $B\left(\bar{v}_{0} ; r\right)$ on the left to a state in $B\left(\bar{u}_{0} ; r\right)$ on the right is a 2-shock having speed greater than 2, satisfying Lemma 1 for small interactions in $\mathfrak{D}$, and such that $\lambda_{1}>2$ in $B\left(\bar{v}_{0} ; r\right)$,

- any strong shock joining a state from $B\left(\bar{v}_{0} ; r\right)$ to a state in $B\left(\bar{u}_{0} ; r\right)$ has a speed greater than (and separated from) all the 1-characteristic speeds in $B\left(\bar{v}_{0} ; r\right)$.

Note that it is easy to fulfill the last condition, since the strong 2-shock obtained above satisfies Lax's inequalities.

Remark 3. The choice of 2 as a minimum for characteristic speeds and for the speed of a strong shock is arbitrary, and could be replaced by any positive constant. Hence, when $\lambda_{1}\left(\bar{u}_{0}\right) \geq 0$, the strong shock that we consider can be arbitrarily small (but of course $r$ becomes very small as well, and the time of controllability is affected). Note also that, as will be clear from the proofs, we could have chosen, instead of a strong 2-shock on the left of the domain, a strong 1-shock on the right of the domain. (In that case we need negative characteristic speeds and negative speed for the strong shock.) Hence the same remark applies when $\lambda_{2}\left(\bar{u}_{0}\right) \leq 0$.

\subsection{Proof of Proposition 2}

In this subsection, we construct a solution with a strong shock, by means of a fronttracking algorithm (see [8]). Let us underline that the results in this subsection are essentially not new: see for instance [32] for a general theory concerning strong waves, [13] where the construction uses Glimm's scheme, and [31] where the construction uses the Bressan-Schochet front-tracking scheme. We describe the construction to make the paper self-contained. Let us describe the algorithm we use. For $n \in \mathbb{N}$ we approximate the initial condition $U_{0}$ with a step function $\mathcal{U}_{n}$ in such a way that

$$
\begin{aligned}
& \mathcal{U}_{n}(x)=\bar{v}_{0} \quad \text { for } x<0 \quad \text { and } \quad \mathcal{U}_{n}(x)=\bar{u}_{0} \quad \text { for } x>1 \text {, } \\
& T V\left(\mathcal{U}_{n}\right) \leq T V\left(U_{0}\right), \quad\left\|\mathcal{U}_{n}-U_{0}\right\|_{L^{1}[0,1]} \rightarrow 0 \quad \text { as } n \rightarrow \infty \text {. }
\end{aligned}
$$

Now starting from $\mathcal{U}_{n}$, we solve (approximately) the various Riemann problems at each discontinuity of $\mathcal{U}_{n}$, and replace each rarefaction wave by a rarefaction fan with accuracy 
$1 / n$, that is, we replace a rarefaction wave between $\omega_{1}$ and $\omega_{2}=\Phi_{i}\left(\sigma, \omega_{1}\right), \sigma>0$, by a piecewise constant solution consisting of constant states:

$$
\omega^{0}:=\omega_{1}, \quad \omega^{k}:=\Phi_{i}\left(k / n, \omega_{1}\right) \quad \text { for } k=1, \ldots, m:=\lfloor n \sigma\rfloor, \quad \omega^{m+1}:=\omega_{2},
$$

separated by straight lines at shock speed $s\left(\omega^{k}, \omega^{k+1}\right)$.

Note that all these Riemann problems are solvable (including the one at $x=0$ ) without vacuum, by Lax's theorem or by Lemma 1 as long as the states lie in the balls described above. By modifying the speeds of the front by an amount of at most $2^{-n}$, one can require that the interactions between fronts are all binary, and that there is at most one interaction at a time. We do not modify the speed of the 2-shock wave issuing from 0.

When two fronts meet, say at time $t_{i}$, let $u_{l}$ to $u_{m}$ be the states separated by the left front, and $u_{m}$ to $u_{r}$ the states separated by the right one; we extend the solution to $t>t_{i}$ by the approximate solution of the Riemann problem, with the following convention for outgoing rarefactions:

- if the incoming waves are of different families, then (possible) outgoing rarefaction waves are approximated by a single rarefaction front,

- if the two incoming waves are of the same family, then the outgoing wave of the other family is approximated by a rarefaction fan with accuracy $1 / n$ as described in (53) if it is a rarefaction, and by a single shock otherwise.

We define the strong shock (denoted by $S$ ) to be the 2-shock that outgoes from 0 , and then the 2-wave (which, as we will show, is still a shock) that extends it after successive interactions. It is unique by the previous conventions. We call any other wave weak. We denote by $U^{n}$ the resulting front-tracking approximation.

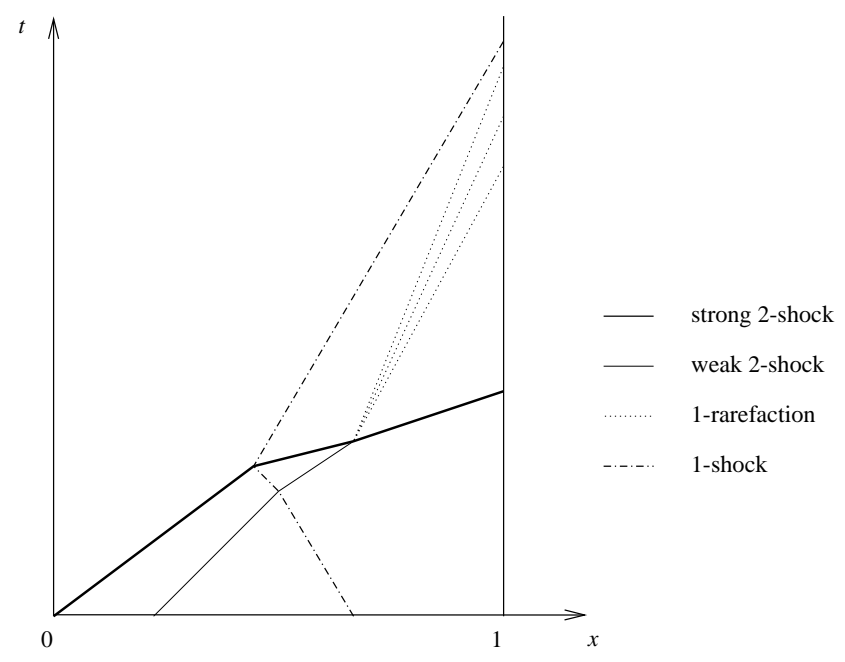

Fig. 3. A front-tracking approximation. 
Now, we prove that the previous algorithm is well defined, and then that one can extract a limit from it which is an entropy solution satisfying the requirements of Proposition 1 .

Estimates on the front-tracking approximations. We introduce the strength $\sigma_{\alpha}$ of a wave $\alpha$ as described in Subsection 2.3. We introduce the following functionals measuring the strength of the solution:

$$
V_{w}(\tau)=\sum_{\alpha \text { weak wave }}\left|\sigma_{\alpha}\right|, \quad V_{s}(\tau)=|S(\tau)| ; \quad V(\tau)=V_{w}(\tau)+V_{S}(\tau)
$$

where $S$ denotes the strong wave, and the sum is over all weak waves existing at time $\tau$. We introduce the following interaction potentials:

$$
Q_{w w}(\tau)=\sum_{\substack{\alpha, \beta \\ \text { approaching } \\ \text { weak waves }}}\left|\sigma_{\alpha}\right| \cdot\left|\sigma_{\beta}\right|, \quad Q_{w s}(\tau)=\sum_{\substack{\alpha \text { weak wave } \\ \text { approaching } \\ \text { the strong wave }}}\left|\sigma_{\alpha}\right|,
$$

where $\alpha$ and $\beta$ are approaching waves with $\alpha$ on the left of $\beta$ and either $\alpha$ is a 2-wave and $\beta$ a 1-wave, or both waves are of the same family and at least one of them is a shock. We introduce the following total interaction potential:

$$
Q(\tau)=\mathcal{A} Q_{w w}(\tau)+Q_{w s}(\tau)
$$

where $\mathcal{A}$ is a constant to be defined.

Clearly, at times when no interaction takes place, all these quantities are constant. Now we describe how they evolve beyond an interaction time. When $\tau$ is a time of interaction and $F$ one of the above quantities, we write $F\left(\tau^{-}\right)$for the constant value of this quantity for $t<\tau$ close to $\tau$, and define $F\left(\tau^{+}\right)$similarly.

The following estimates are valid as long as all states on the left of $S$ are in $B\left(\bar{v}_{0}, r\right)$ and all states on the right of $S$ are in $B\left(\bar{u}_{0}, r\right)$, which will be proved a posteriori provided $T V\left(u_{0}\right)$ is small. All the $O(1)$ in the following interaction estimates are fixed once $r$ introduced before is fixed.

Now it follows from Glimm's estimates (see [21]) that, during a weak-weak interaction (call the corresponding waves $\alpha$ and $\beta$ ):

$$
\left\{\begin{array}{l}
V_{w}\left(\tau^{+}\right)-V_{w}\left(\tau^{-}\right)=O(1)\left|\sigma_{\alpha}\right| \cdot\left|\sigma_{\beta}\right| \quad \text { and } \quad V_{s}\left(\tau^{+}\right)=V_{s}\left(\tau^{-}\right), \\
Q_{w w}\left(\tau^{+}\right)-Q_{w w}\left(\tau^{-}\right)=-\left|\sigma_{\alpha}\right| \cdot\left|\sigma_{\beta}\right|+O(1)\left|\sigma_{\alpha}\right| \cdot\left|\sigma_{\beta}\right| V_{w}\left(\tau^{-}\right), \\
Q_{w s}\left(\tau^{+}\right)-Q_{w s}\left(\tau^{-}\right)=O(1)\left|\sigma_{\alpha}\right| \cdot\left|\sigma_{\beta}\right| .
\end{array}\right.
$$

Following Corollary 1 during a weak-strong interaction (call the corresponding waves $\alpha$ and $S$ respectively), we have the following estimates:

$$
\left\{\begin{array}{l}
V_{w}\left(\tau^{+}\right)-V_{w}\left(\tau^{-}\right)=O(1)\left|\sigma_{\alpha}\right|, \quad V_{s}\left(\tau^{+}\right)-V_{s}\left(\tau^{-}\right)=O(1)\left|\sigma_{\alpha}\right|, \\
Q_{w w}\left(\tau^{+}\right)-Q_{w w}\left(\tau^{-}\right)=O(1)\left|\sigma_{\alpha}\right| V_{w}\left(\tau^{-}\right), \quad Q_{w s}\left(\tau^{+}\right)-Q_{w s}\left(\tau^{-}\right)=-\left|\sigma_{\alpha}\right| .
\end{array}\right.
$$


It follows from 57 that for suitable $\mathcal{A}$, during a weak-weak interaction one has

$$
Q\left(\tau^{+}\right)-Q\left(\tau^{-}\right)=-\frac{\mathcal{A}}{2}\left|\sigma_{\alpha}\right|\left|\sigma_{\beta}\right|+O(1) \mathcal{A}\left|\sigma_{\alpha}\right|\left|\sigma_{\beta}\right| V_{w}\left(\tau^{-}\right),
$$

and during a strong-weak interaction, from 58,

$$
Q\left(\tau^{+}\right)-Q\left(\tau^{-}\right)=-\left|\sigma_{\alpha}\right|+O(1) \mathcal{A}\left|\sigma_{\alpha}\right| V_{w}\left(\tau^{-}\right) .
$$

Now one sees that there exists a constant $c_{1}>0$ such that if $V_{w}\left(\tau^{-}\right)<c_{1}$, then

- during a weak-weak interaction:

$$
Q_{w w}\left(\tau^{+}\right)-Q_{w w}\left(\tau^{-}\right) \leq-\frac{\left|\sigma_{\alpha}\right|\left|\sigma_{\beta}\right|}{2} \text { and } Q\left(\tau^{+}\right)-Q\left(\tau^{-}\right) \leq-\frac{\mathcal{A}}{2}\left|\sigma_{\alpha}\right|\left|\sigma_{\beta}\right|,
$$

- during a strong-weak interaction:

$$
Q_{w s}\left(\tau^{+}\right)-Q_{w s}\left(\tau^{-}\right)=-\left|\sigma_{\alpha}\right|, \quad Q\left(\tau^{+}\right)-Q\left(\tau^{-}\right) \leq-\left|\sigma_{\alpha}\right| / 2 .
$$

One deduces that there is a constant $C_{1}$ such that, provided $V_{w}(\tau)$ stays below $c_{1}$,

$$
\begin{gathered}
\tau \mapsto V_{w}(\tau)+C_{1} Q(\tau) \text { and } \tau \mapsto V_{S}(\tau)+C_{1} Q(\tau) \text { are nonincreasing, } \\
\tau \mapsto V_{s}(\tau)-C_{1} Q(\tau) \text { is nondecreasing. }
\end{gathered}
$$

Moreover, if $V_{w}(0)$ is small enough (say $V_{w}(0)<c_{2}$ ), the latter quantity is positive.

Now if $V_{w}(0)$ is small enough, one can get $V_{w}(\tau)<c_{1}$ for all $\tau$ (at least as long as the front-tracking approximation is well-defined, which is proven to be globally later), and hence 63 -64 hold for all time. Indeed, introduce $L>0$ such that

$$
\frac{1}{L} T V_{\Sigma^{ \pm}(t)}\left(U^{n}(t)\right) \leq V_{w}(t) \leq L \cdot T V_{\Sigma^{ \pm}(t)}\left(U^{n}(t)\right)
$$

where $\Sigma^{+}(t)$ (resp. $\left.\Sigma-(t)\right)$ is the (open) part of $\mathbb{R}$ on the right (resp. left) of the strong shock, and $\Sigma^{ \pm}(t):=\Sigma^{+}(t) \cup \Sigma^{+}(t)$. (Clearly one has 65) for each small front in the neighborhoods of $\bar{v}_{0}$ and $\bar{u}_{0}$. Hence the constant $L$ depends on $r$.) Suppose $T V_{\mathbb{R}} * \mathcal{U}_{n}<c_{2}$, for some $c_{2}<c_{1} / L$ to be fixed later. This remains true for $U^{n}(\tau)$ for $\tau$ close to 0 . By (63) one has

$$
V_{w}(\tau) \leq V_{w}(0)+C_{1} Q(0) \leq L c_{2}+C_{1} L^{2} c_{2}^{2}
$$

and hence

$$
T V_{\Sigma^{ \pm}(t)}\left(U^{n}(t)\right) \leq L^{2} c_{2}+C_{1} L^{3} c_{2}^{2} .
$$

Now we choose $c_{2}$ that satisfies $c_{2}<c_{1} / L, L c_{2}+C_{1} L^{2} c_{2}^{2}<c_{1}$ and $L^{2} c_{2}+C_{1} L^{3} c_{2}^{2}<r$. This ensures that, at least as long as the front-tracking approximation is well-defined, all the previous estimates are valid.

Finite number of fronts and nonaccumulation of interaction points. In order to prove that the front-tracking approximation is indeed well-defined, it remains to prove that the total number of fronts is finite and that interaction points do not accumulate. It follows 
from the construction that, at each interaction for which the number of outgoing fronts exceeds the one of incoming fronts (that is, two), the strength of the outgoing rarefaction wave exceeds $1 / n$. Using Glimm's interaction estimate, one deduces that the strengths of the incoming fronts $\sigma$ and $\sigma^{\prime}$ satisfy $|\sigma|\left|\sigma^{\prime}\right| \geq C / n$, and hence, using (61) and (62), one sees that this can happen only a finite number of times. Consequently, the total number of fronts is finite. It is then rather classical that interaction points cannot accumulate (see for instance [1]): first, we can restrict to the case where interactions generate only two fronts (by considering a suitable time interval); then one can for instance reason by induction on the number of fronts "involved" in an accumulation of fronts. Hence the above fronttracking algorithm is well-defined, for any $n \in \mathbb{N}^{*}$.

Size of the rarefaction fronts. Classically, before passing to the limit and getting a solution of $(\mathrm{EI})$, we need an estimate on the size of rarefaction fronts, which is central in the proof of the entropy inequality. We consider a rarefaction front $v$ (which starts at time $\underline{\tau} \geq 0$ ). This is done as in the small total variation case (see [8]). By the convention we made in the resolution of interactions, it is quite clear that if a $j$-wave is involved in an interaction, there is at most one outgoing $j$-wave. Hence, a $j$-wave can be uniquely extended for $t \geq \underline{\tau}$ by following the outgoing $j$-wave at each interaction (with possibly a final time $\bar{\tau}<+\infty)$.

Clearly, at the beginning, the size of $v$ satisfies $\left|\sigma_{v}(\underline{\tau})\right| \leq 1 / n$. Then one introduces the total strength of waves approaching $v$ :

$$
V_{v}(\tau)=\sum_{\mu \text { approaching } v}\left|\sigma_{\mu}\right|
$$

where the summation involves both strong and weak waves. By considering the different possible types of interactions, one finds that for $T V\left(u_{0}\right)$ small enough and for some $c_{3}>0$,

$$
\left|\sigma_{v}\left(\tau^{+}\right)\right| \leq\left|\sigma_{v}\left(\tau^{-}\right)\right|\left[1-c_{3}\left\{\left(V_{v}\left(\tau^{+}\right)+C_{1} Q\left(\tau^{+}\right)\right)-\left(V_{v}\left(\tau^{-}\right)+C_{1} Q\left(\tau^{-}\right)\right)\right\}\right],
$$

which leads to

$$
\begin{aligned}
\left|\sigma_{v}(t)\right| & \leq\left|\sigma_{v}(\underline{\tau})\right| \prod_{\begin{array}{c}
\mu \operatorname{crossing} v \\
\text { at time } \tau \in[\underline{\tau}, t]
\end{array}}\left[1-c_{3}\left\{\left(V_{v}\left(\tau^{+}\right)+C_{1} Q\left(\tau^{+}\right)\right)-\left(V_{v}\left(\tau^{-}\right)+C_{1} Q\left(\tau^{-}\right)\right)\right\}\right] \\
& \leq\left|\sigma_{v}(\underline{\tau})\right| \exp \left(c_{3}\left[V(0)+C_{1} Q(0)\right] \leq C_{3} / n\right.
\end{aligned}
$$

(as long as $v$ is a rarefaction front).

Passing to the limit. Now we show that one can find a converging subsequence of the family $U^{n}$ as $n \rightarrow \infty$. We already saw that $\left(U^{n}\right)_{n \in \mathbb{N}}$ has a uniformly bounded total variation for fixed $\tau$, outside the strong shock. The strength of the shock is measured by $V_{S}(\tau)$, which can be estimated by (63); hence the total variation is uniformly bounded. We denote by $\mathcal{V}$ a strict bound for all front speeds in various $U^{n}$ (for instance the supremum 
of the characteristic speeds over $B\left(U_{0} ; r\right) \cup B\left(\bar{u}_{0} ; r\right)$ plus 1$)$. Then, classically, the family is uniformly Lipschitz in time with values in $L_{\text {loc }}^{1}\left(\mathbb{R} ; \mathbb{R}^{+*} \times \mathbb{R}\right)$ :

$$
\left\|U^{n}(\tau+h)-U^{n}(\tau)\right\|_{L^{1}([M, M])} \leq(t-s) \mathcal{V} \max _{[\tau, \tau+h]} T V\left(U^{n}(s)\right),
$$

at least when $[\tau, \tau+h]$ does not contain an interaction time, and then by continuity for any time interval. Of course, the maximum on the right hand side is bounded. Hence, by Helly's theorem (see e.g. [8, Theorem 2.4]), one can extract a subsequence, still denoted by $U^{n}$, such that

$$
U^{n} \rightarrow \bar{U} \quad \text { in } L_{\mathrm{loc}}^{1}([0,+\infty) \times \mathbb{R}) .
$$

Proof that $\bar{U}$ is an entropic solution. This is done as in [8, pp. 144-145], and recalled in Subsection 5.9 for completeness.

Proof that $\bar{U}_{\mid t=1}$ is constant in $[0,1]$. Let us prove that for $n$ large enough, all the fronttracking approximations $U^{n}$ are constant in $[2 / 3,1] \times[0,1]$ (at least if $T V\left(u_{0}\right)$ has been chosen small enough). To do this, we prove that all waves on the left of the strong shock (including the strong shock) evolve with speed at least $3 / 2$.

Weak waves on the left of the strong shock. It follows from (63) and (65) that if $V_{w}(0)$ is small enough,

$$
V_{\mid \Sigma^{-}(\tau)}\left(U^{n}\right) \leq \varepsilon
$$

As by construction $\bar{v}_{0}$ is the constant state of $U_{n}$ as $x \rightarrow-\infty$, one finds that for any $x \in \Sigma^{-}(t)$,

$$
\lambda_{2}\left(U^{n}(x)\right) \geq \lambda_{1}\left(U^{n}(x)\right) \geq 2 .
$$

Now, using [32), one deduces that all fronts move with speed at least $3 / 2$, if $V_{w}(0)$ is small enough, also after taking into account the small changes in the front speeds of order $2^{-n}$.

The strong shock. It follows from the construction that the state on the left of the strong shock is at distance at most $r>0$ from $\bar{v}_{0}$, whereas the state on the right of it is at distance at most $r>0$ from $\bar{u}_{0}$. By the definition of $r$ in Subsection 3.2 this implies that the strong shock evolves with speed greater than 2 .

This shows that the restriction of $U^{n}$ to the space interval $[0,1]$ is constant for times $\tau \geq 2 / 3$. So the limit $\bar{U}$ (which is Lipschitz with values in $L_{\text {loc }}^{1}$ ) is constant in $[0,1]$ at times $\tau \geq 2 / 3$.

\section{Step 2: from a constant state to another}

The goal of this section is to prove the following proposition:

Proposition 3. For any $\left(\omega, \omega^{\prime}\right) \in\left(\mathbb{R}^{+*} \times \mathbb{R}\right)^{2}$, there is some $T_{2}>0$ and an entropy solution $u$ of $(\mathrm{EI})$ in $[0, T] \times[0,1]$ such that

$$
\begin{gathered}
u_{\mid t=0}=\omega, \\
u_{\mid t=T_{2}}=\omega^{\prime} .
\end{gathered}
$$

This proposition is proven in three steps, which we develop in separate subsections. 


\subsection{Traveling between zones}

Let us prove the following lemma:

Lemma 3. For all $\omega_{0} \in \mathbb{R}^{+*} \times \mathbb{R}$, for any $i \in\{1,2,3\}$, there exists $T_{2}^{1}>0$ and an entropy solution $u$ of $(\mathrm{EI})$ in $\left[0, T_{2}^{1}\right] \times[0,1]$ such that

$$
\begin{gathered}
u_{\mid t=0}=\omega_{0}, \\
u_{\mid t=T_{2}^{1}} \in \mathcal{D}_{i} .
\end{gathered}
$$

Proof. We separate cases:

Case 1: $\omega_{0} \in \mathcal{D}_{1} \cup \mathcal{C}_{1}$ and $i \in\{2,3\}$.

Subcase $(i): i=2$. Starting from $\omega_{0}=\left(\rho_{0}, m_{0}\right)$, we follow the 1 -shock curve until it crosses the line $m=0$. In other words, we determine $\omega_{1}=\left(\rho_{1}, m_{1}\right)$ such that

$$
\left(\omega_{0},\left(\rho_{1}, m_{1}\right)\right) \text { is a } 1 \text {-shock and } m_{1}=0 \text {. }
$$

It is quite clear that:

- the speed of the corresponding shock is negative, thanks to (28) and (30);

- such a point $\left(\rho_{1}, 0\right)$ exists because $m_{0} \geq \kappa \rho_{0}^{\gamma}>0$, and it follows from 30 that, as $\rho \rightarrow+\infty, \tilde{\Phi}_{1}\left(\rho, \omega_{0}\right) \rightarrow-\infty$.

Then the restriction to the domain $[0,1]$ of the solution of the Riemann problem with initial data $\omega_{0}$ for $x<1$ and $\omega_{1}$ for $x>1$ is as required.

Subcase (ii): $i=3$ : Again we choose $\omega_{1}=\left(\rho_{1}, m_{1}\right)=\left(\rho_{1}, \tilde{\Phi}_{1}\left(\rho_{1}, \omega_{0}\right)\right)$ for some $\rho_{1}>\rho_{0}$ large enough. It is a consequence of 21$)$ and 30 that $\lambda_{2}\left(\rho, \tilde{\Phi}_{1}\left(\rho, \omega_{0}\right)\right) \rightarrow-\infty$ as $\rho \rightarrow+\infty$. Hence for $\rho$ large enough, one has $\omega_{1} \in \mathcal{D}_{3}$. One concludes as in the previous subcase.

Case 2: $\omega_{0} \in \mathcal{D}_{2} \cup \mathcal{C}_{2}$ and $i=3$. This case can be treated exactly as case 1 , subcase (ii).

Case 3: $\omega_{0} \in \mathcal{D}_{3} \cup \mathcal{C}_{2}$ and $i \in\{1,2\}$. This case can be treated exactly as case 1 , but here the 1 -shock is replaced by a 2 -shock on the left, that is, one finds $\omega_{1}$ such that the Riemann problem $\left(\omega_{1}, \omega_{0}\right)$ is solved in terms of a 2 -shock. Then the desired solution is given by the solution of the Riemann problem with initial data $\omega_{1}$ for $x<0$ and $\omega_{0}$ for $x>0$.

Case 4: $\omega_{0} \in \mathcal{D}_{2} \cup \mathcal{C}_{1}$ and $i=1$. This case can be treated exactly as case 1 , subcase (ii), with again the 1 -shock replaced by a left 2 -shock.

\subsection{Traveling between points inside a zone}

Let us prove the following lemma: 
Lemma 4. Let $E \in\left\{\mathcal{D}_{1}, \mathcal{D}_{2}, \mathcal{D}_{3}\right\}$. For all $\omega_{0}, \omega_{1} \in E$, there exists $T_{2}^{2}>0$ and an entropy solution $u$ of $\sqrt{\mathrm{EI}}$ in $\left[0, T_{2}^{2}\right] \times[0,1]$ such that

$$
\begin{gathered}
u_{\mid t=0}=\omega_{0}, \\
u_{\mid t=T_{2}^{2}}=\omega_{1} .
\end{gathered}
$$

Proof. We first assume that we can solve the problem locally, that is, given $\tilde{\omega}$, there is a neighborhood $\mathcal{V}$ of $\tilde{\omega}$ in $E$ such that any $\check{\omega}$ in $\mathcal{V}$ can be reached as in the above lemma. Then this can be done globally, that is, for any $\breve{\omega}$ in $E$. Indeed, given two states in $E$, one considers a smooth arc $\Gamma:[0,1] \rightarrow E$ which joins $\omega_{0}$ to $\omega_{1}$ (it is straightforward to see that $\mathcal{D}_{i}$ is arc-connected for any $i$ ). Then, since the local problem is solved, there is $r_{x}>0$ in which any state is attainable in finite time by an entropic solution starting at $x$. Since $\Gamma$ is covered by the union of the balls $B\left(x, r_{x} / 2\right)$, there are a finite number of real numbers $0=t_{0}<t_{1}<\cdots<t_{n}=1$ such that $\Gamma\left(t_{0}\right)=\omega_{0}, \Gamma\left(t_{n}\right)=\omega_{1}$, and $\Gamma\left(t_{i+1}\right)$ can be attained from $\Gamma\left(t_{i}\right)$ by an entropy solution, which yields a solution.

Concerning the local problem, we separate cases:

Case 1: $E=\mathcal{D}_{1}$. Consider $\tilde{\omega}$ in $\mathcal{D}_{1}$. Then for $\check{\omega}$ close enough to $\tilde{\omega}$, the different states in the solution of the Riemann problem $(\tilde{\omega}, \check{\omega})$ are all in $\mathcal{D}_{1}$. Then the solution $u$ of the Riemann problem with initial value $\tilde{\omega}$ for $x<0$ and $\check{\omega}$ for $x>0$ is as desired since all the waves have positive speed.

Case 2: $E=\mathcal{D}_{3}$. This case can be treated exactly as case 1, except that one considers the solution of the Riemann problem for initial data $\tilde{\omega}$ for $x<1$ and $\check{\omega}$ for $x>1$.

Case 3: $E=\mathcal{D}_{2}$. Starting from a state $\tilde{\omega}$, one can shift it (at least locally) to another state belonging to:

- the 1-rarefaction curve starting from $\tilde{\omega}$ : given $\check{\omega}$ on that curve, one considers the solution of the Riemann problem with initial data $\tilde{\omega}$ for $x<1$ and $\check{\omega}$ for $x>1$,

- the 1-shock curve starting from $\tilde{\omega}$ : given $\check{\omega}$ on that curve, one considers the solution of the Riemann problem with initial data $\tilde{\omega}$ for $x<1$ and $\check{\omega}$ for $x>1$ (because the speed of the shock in that case is clearly negative),

- the 2-rarefaction curve on the left starting from $\tilde{\omega}$ : given $\check{\omega}$ on that curve, one considers the solution of the Riemann problem with initial data $\check{\omega}$ for $x<0$ and $\tilde{\omega}$ for $x>0$,

- the 2-shock curve on the left starting from $\tilde{\omega}$ : given $\check{\omega}$ on that curve, one considers the solution of the Riemann problem with initial data $\check{\omega}$ for $x<0$ and $\tilde{\omega}$ for $x>0$.

One concludes essentially as for Lax's theorem on existence of a solution to the Riemann problem when the two states are close: at the point $\tilde{\omega}$, one considers the curve $\Phi_{1}$ consisting of states that are connected to $\tilde{\omega}$ by either shock or rarefaction, when put on the right of $\tilde{\omega}$, and the curve $\bar{\Phi}_{2}$ consisting of states that are connected to $\tilde{\omega}$ by either shock or rarefaction, when put on the left of $\tilde{\omega}$. By the local inversion theorem, in a neighborhood of $\tilde{\omega}$, one can find for any $\check{u}$ some real numbers $\sigma_{1}$ and $\sigma_{2}$ such that $\check{u}=\Phi_{2}^{l}\left(\sigma_{2}, \Phi_{1}\left(\sigma_{1}, \tilde{u}\right)\right.$ ). Hence one reaches $\breve{u}$ by first letting a 1-wave pass through the domain from right to left, then by letting a 2 -wave pass through the domain from left to right. 


\subsection{Reaching a critical curve}

Let us prove the following lemma:

Lemma 5. For all $\omega_{1} \in \mathcal{C}_{1} \cup \mathcal{C}_{2}$, there are $\omega_{0} \in \mathcal{D}_{2}, T_{2}^{3}>0$ and an entropic solution $u$ of $\mathrm{EI}$ in $\left[0, T_{2}^{3}\right] \times[0,1]$ such that

$$
\begin{gathered}
u_{\mid t=0}=\omega_{0}, \\
u_{\mid t=T_{2}^{3}}=\omega_{1} .
\end{gathered}
$$

Proof. We only consider the case $\omega_{1} \in \mathcal{C}_{1}$ since the proof in the case $\omega_{1} \in \mathcal{C}_{2}$ is identical. In that case, we seek a proper $\omega_{0}$ on the left 2-rarefaction curve. In Riemann invariant coordinates, along that curve,

$$
w^{1}=\frac{m}{\rho}+\frac{2 \sqrt{\kappa \gamma}}{\gamma-1} \rho^{(\gamma-1) / 2}
$$

decreases, whereas

$$
w^{2}=\frac{m}{\rho}-\frac{2 \sqrt{\kappa \gamma}}{\gamma-1} \rho^{(\gamma-1) / 2}
$$

is constant. But

$$
\lambda_{1}(\rho, m)=\frac{m}{\rho}-\kappa \rho^{(\gamma-1) / 2}=\left(\frac{1}{2}-\frac{\gamma-1}{4 \sqrt{\gamma}}\right) w^{1}+\left(\frac{1}{2}+\frac{\gamma-1}{4 \sqrt{\gamma}}\right) w^{2} .
$$

Since $0 \leq \frac{\gamma-1}{4 \sqrt{\gamma}} \leq \frac{1}{2}$, this implies that $\lambda_{1}$ decreases on that curve, and hence one finds $\omega_{0} \in \mathcal{D}_{2}$ (close to $\left.\omega_{1}\right)$ such that the Riemann problem $\left(\omega_{0}, \omega_{1}\right)$ is solved by a 2-rarefaction wave (with positive speed). Hence, the restriction of this solution of the Riemann problem to $[0,1]$ is as desired.

\subsection{Conclusion}

One sees that, to prove Proposition 3 , one can restrict by Lemma 5 to the case $\omega^{\prime} \in$ $\mathcal{D}_{1} \cup \mathcal{D}_{2} \cup \mathcal{D}_{3}$. By Lemma 3 , we can restrict to the case where $\omega$ and $\omega^{\prime}$ belong to the same $\mathcal{D}_{i}$. This is exactly Lemma 4. Note that in this section one can generate solutions with arbitrarily small total variation, by taking sufficiently numerous and small steps (but this is costly in time).

\section{Step 3: attaining the final state}

\subsection{Introduction}

The goal of this section is to prove the following proposition: 
Proposition 4. Let $\bar{u}_{1}=\left(\bar{\rho}_{1}, \bar{m}_{1}\right)$ be a constant state in $\mathbb{R}^{+*} \times \mathbb{R}$, with characteristic speeds $\bar{\lambda}_{1}:=\lambda_{1}\left(\bar{\rho}_{1}, \bar{m}_{1}\right)$ and $\bar{\lambda}_{2}:=\lambda_{2}\left(\bar{\rho}_{1}, \bar{m}_{1}\right)$. Introduce

$$
\bar{\xi}_{1}:=\frac{\partial \lambda_{1}}{\partial w^{2}}\left(\bar{u}_{1}\right) \quad \text { and } \quad \bar{\xi}_{2}:=\frac{\partial \lambda_{2}}{\partial w^{1}}\left(\bar{u}_{1}\right) .
$$

Then there exists $T_{3}=T_{3}\left(\bar{u}_{1}\right)>0$ such that for any $\alpha>0$, there exists $\varepsilon>0$ such that, for any $u_{1} \in B V\left([0,1] ; \mathbb{R}^{+*} \times \mathbb{R}\right)$ satisfying:

$$
\begin{aligned}
& \forall x, y \in[0,1], x<y, \\
& \quad \frac{w^{2}\left(u_{1}(x)\right)-w^{2}\left(u_{1}(y)\right)}{x-y} \leq(1-\alpha) \frac{1}{\bar{\xi}_{1}} \max \left(\frac{\bar{\lambda}_{2}-\bar{\lambda}_{1}}{1-y}, \frac{\bar{\lambda}_{1}}{x}, \frac{-\bar{\lambda}_{1}}{1-y}\right), \\
& \forall x, y \in[0,1], x<y, \\
& \frac{w^{1}\left(u_{1}(x)\right)-w^{1}\left(u_{1}(y)\right)}{x-y} \leq(1-\alpha) \frac{1}{\bar{\xi}_{2}} \max \left(\frac{\bar{\lambda}_{2}-\bar{\lambda}_{1}}{x}, \frac{-\bar{\lambda}_{2}}{1-y}, \frac{\bar{\lambda}_{2}}{x},\right), \\
& \left\|u_{1}-\bar{u}_{1}\right\|_{L^{\infty}([0,1])}<\varepsilon \text { and } T V\left(u_{1}\right)<\varepsilon,
\end{aligned}
$$

there exists $\omega \in \mathbb{R}^{+*} \times \mathbb{R}$ and an entropic solution $u$ of $\sqrt{\mathrm{EI}}$ in $\left[-T_{3}, 0\right] \times[0,1]$ such that

$$
\begin{aligned}
u_{\mid t=-T_{3}} & =\omega, \\
u_{\mid t=0} & =u_{1} .
\end{aligned}
$$

Remark 4. In $[$ EI $)$, one finds

$$
\bar{\xi}_{1}=\bar{\xi}_{2}=c_{\gamma}^{-1}=\frac{1}{2}+\frac{\gamma-1}{4 \sqrt{\gamma}} .
$$

We keep this notation in order that the proof of this section can be easily adapted to the system $(\mathrm{P})$.

The proof of Proposition 4 is by constructing the solution via a (backward) fronttracking algorithm. As in Section 3 , the idea is to consider as a final state a function defined on $\mathbb{R}$ and composed of $u_{1}$ in $[0,1]$, and constant states to the left of 0 and to the right of 1 , which are separated from $u_{1}(0)$ and $u_{1}(1)$ respectively by strong shocks whose sizes depend on $\alpha$. According to the position of $\bar{u}_{1}$ in $\mathbb{R}^{+*} \times \mathbb{R}$, one may consider only one shock instead of two. This is developed in the next subsection. The front-tracking algorithm used here is divided into four main steps:

- approximation of the final state,

- description of the algorithm,

- estimates and well-posedness of the algorithm,

- convergence and validity of the limit. 


\subsection{Extension of the final state}

We first define the strong shocks that are to enter on both sides of the domain. Possibly, only one shock will be considered. Given $\alpha$, there are two states $\mathfrak{u}_{1}^{-}$and $\mathfrak{u}_{1}^{+}$such that:

$$
\begin{aligned}
& \left(\mathfrak{u}_{1}^{-}, \bar{u}_{1}\right) \text { is a } 1 \text {-shock of speed } \varsigma_{1} \text { with } \bar{\lambda}_{1}+\beta / 2 \leq \varsigma_{1} \leq \bar{\lambda}_{1}+\beta, \\
& \left(\bar{u}_{1}, \mathfrak{u}_{1}^{+}\right) \text {is a } 2 \text {-shock of speed } \varsigma_{2} \text { with } \bar{\lambda}_{2}-\beta \leq \varsigma_{2} \leq \bar{\lambda}_{2}-\beta / 2 .
\end{aligned}
$$

The constant $\beta$ will be fixed in terms of $\alpha$ later (at the end of Subsection 5.8. We also have the following constraints on the shock:

- if $\bar{u}_{1} \in \mathcal{D}_{1} \cup \mathcal{C}_{1}$, we ask that $\mathfrak{u}_{1}^{+} \notin \mathcal{C}_{1} \cup \mathcal{C}_{2}$,

- if $\bar{u}_{1} \in \mathcal{D}_{3} \cup \mathcal{C}_{2}$, we ask that $\mathfrak{u}_{1}^{-} \notin \mathcal{C}_{1} \cup \mathcal{C}_{2}$,

- if $\bar{u}_{1} \in \mathcal{D}_{2}$, we ask that the interaction (in decreasing time) of the 1-shock $\left(\mathfrak{u}_{1}^{-}, \bar{u}_{1}\right)$ and the 2 -shock $\left(\bar{u}_{1}, \mathfrak{u}_{1}^{+}\right)$generate a 2 -shock $\left(\mathfrak{u}_{1}^{-}, \tilde{u}_{1}\right)$ and a 1 -shock $\left(\tilde{u}_{1}, \mathfrak{u}_{1}^{+}\right)$of respective speeds $\varsigma_{2}^{\prime}$ and $\varsigma_{1}^{\prime}$ satisfying

$$
\varsigma_{1}^{\prime}<\lambda_{1}\left(\tilde{u}_{1}\right) \leq-c_{1}<0 \quad \text { and } \quad \varsigma_{2}^{\prime}>\lambda_{2}\left(\tilde{u}_{1}\right) \geq c_{1}>0
$$

This means that $\mathfrak{u}_{1}^{+}=\Phi_{2}\left(\sigma_{1}, \Phi_{1}\left(\sigma_{2}, \mathfrak{u}_{1}^{-}\right)\right)$with $\sigma_{1}, \sigma_{2}<0$ and also $\mathfrak{u}_{1}^{+}=\Phi_{1}\left(\sigma_{2}^{\prime}\right.$, $\left.\Phi_{2}\left(\sigma_{1}^{\prime}, \mathfrak{u}_{1}^{-}\right)\right)$with $\sigma_{1}^{\prime}, \sigma_{2}^{\prime}<0$ and $\tilde{u}_{1}:=\Phi_{2}\left(\sigma_{1}^{\prime}, \mathfrak{u}_{1}^{-}\right)$satisfying the above conditions.

This is easily obtained at least for small shocks from $\bar{u}_{1}$.

Remark 5. Note that in this section, the strong shocks that we are using can be arbitrarily small. But as these shocks shrink, the constant $\varepsilon_{2}$ that appears in Theorem 1 tends to 0 and the time of controllability possibly tends to $+\infty$ (if $\bar{u}_{1}$ is on a critical curve).

We fix the following notations:

$$
\begin{array}{ll}
\bar{u}_{1}=\Phi_{1}\left(\mu_{1}, \mathfrak{u}_{1}^{-}\right), & \mathfrak{u}_{1}^{+}=\Phi_{2}\left(\mu_{2}, \bar{u}_{1}\right), \\
\tilde{u}_{1}=\Phi_{2}\left(\rho_{2}, \mathfrak{u}_{1}^{-}\right), & \mathfrak{u}_{1}^{+}=\Phi_{1}\left(\rho_{1}, \tilde{u}_{1}\right) .
\end{array}
$$

Note that $u_{1}$ being a $B V([0,1])$ function, it has limits at $0^{+}$and $1^{-}$, which we naturally denote respectively by $u_{1}\left(0^{+}\right)$and $u_{1}\left(1^{-}\right)$.

Now, the shocks are retained according to the following rule:

- If $\bar{u}_{1} \in \mathcal{D}_{1} \cup \mathcal{C}_{1}$, then we retain only the 2-shock; we fix $U_{1}^{-}:=u_{1}\left(0^{+}\right)$and $U_{1}^{+}:=$ $\Phi_{2}\left(\mu_{2}, u_{1}\left(1^{-}\right)\right)$.

- If $\bar{u}_{1} \in \mathcal{D}_{2}$, then we retain both shocks; we fix $U_{1}^{-}$such that $u_{1}\left(0^{+}\right)=\Phi_{1}\left(\mu_{1}, U_{1}^{-}\right)$ and $U_{1}^{+}=\Phi_{2}\left(\mu_{2}, u_{1}\left(1^{-}\right)\right)$.

- If $\bar{u}_{1} \in \mathcal{D}_{3} \cup \mathcal{C}_{2}$, then we retain only the 1-shock; we fix $U_{1}^{-}$such that $u_{1}\left(1^{+}\right)=$ $\Phi_{1}\left(\mu_{1}, U_{1}^{-}\right)$and $U_{1}^{+}=u_{1}\left(1^{-}\right)$. 
In all cases we extend the final state over $\mathbb{R}$ in the following way:

$$
U_{f}(x)= \begin{cases}U_{1}^{-} & \text {for } x<0, \\ u_{1}(x) & \text { for } 0 \leq x \leq 1, \\ U_{1}^{+} & \text {for } x>1\end{cases}
$$

The shocks at 0 and 1 (and their natural descendants) will be called strong shocks, the other waves in the domain will be called weak.

\subsection{Backward interactions}

Before describing our construction, we give some lemmas that will be useful to deal with interactions in the context of a backward front-tracking algorithm. One should bear in mind that there is no uniqueness in extending a solution backward (when it is possible).

There are several types of (backward) interactions that may happen:

\section{Interaction of two weak fronts of opposite families.}

Lemma 6. Consider two states $u_{l}$ and $u_{r}$, both belonging to a neighborhood of $\mathfrak{u}_{1}^{-}, \mathfrak{u}_{1}^{+}$, $\bar{u}_{1}$ or $\tilde{u}_{1}$, and satisfying

$$
u_{r}=\Phi_{2}\left(\sigma_{2}, \Phi_{1}\left(\sigma_{1}, u_{l}\right)\right)
$$

for $\sigma_{1}$ and $\sigma_{2}$ in a neighborhood of 0 . Then for some $\sigma_{1}^{\prime}$ and $\sigma_{2}^{\prime}$,

$$
u_{r}=\Phi_{1}\left(\sigma_{1}^{\prime}, \Phi_{2}\left(\sigma_{2}^{\prime}, u_{l}\right)\right)
$$

with

$$
\left|\sigma_{1}^{\prime}-\sigma_{1}\right|+\left|\sigma_{2}^{\prime}-\sigma_{2}\right| \leq C_{1}\left|\sigma_{1}\right|\left|\sigma_{2}\right| .
$$

Proof. The proof follows exactly the proof of Lax's theorem and Glimm's estimates without changes.

Interaction of a strong shock and a weak shock front of opposite families. We consider a backward interaction of type strong 1-shock/weak 2-shock. The case of weak 1 -shock/strong 2-shock interactions is treated similarly. Also, we only treat the case of a strong shock close to $\left(\mathfrak{u}_{1}^{-}, \bar{u}_{1}\right)$, as the case of the shock $\left(\tilde{u}_{1}, \mathfrak{u}_{1}^{+}\right)$is similar.

Lemma 7. Consider three states $u_{l}, u_{m}$ and $u_{r}$, where $u_{l}$ belongs to a neighborhood of $\mathfrak{u}_{1}^{-}$, while $u_{m}$ and $u_{r}$ are in a neighborhood of $\bar{u}_{1}$. Suppose they satisfy

$$
u_{r}=\Phi_{2}\left(\sigma_{2}, \Phi_{1}\left(\rho_{1}, u_{l}\right)\right), \quad u_{m}=\Phi_{1}\left(\rho_{1}, u_{l}\right),
$$

with $\sigma_{1}, \sigma_{2}<0, \rho_{1}$ in a neighborhood of $\mu_{1}$, and $\sigma_{2}$ in a neighborhood of 0 . Then one can find $\rho_{1}^{\prime}<0$ and $\sigma_{2}^{\prime}<0$ such that

$$
\begin{gathered}
u_{r}=\Phi_{1}\left(\rho_{1}^{\prime}, \Phi_{2}\left(\sigma_{2}^{\prime}, u_{l}\right)\right), \\
\left|\rho_{1}^{\prime}-\rho_{1}\right|+\left|\sigma_{2}^{\prime}\right| \leq C_{2}\left|\sigma_{2}\right| .
\end{gathered}
$$


Proof. The existence of $\rho_{1}^{\prime}$ and $\sigma_{2}^{\prime}$ is proven as in Lemma 1; observing that for a strong 2shock $\left(u_{l}, u_{m}\right)$ the family $\left\{r_{1}\left(u_{m}\right), u_{m}-u_{r}\right\}$ is free (using Lax's inequalities for instance), one finds that $\left(p, \sigma_{2}\right) \mapsto \Phi_{1}\left(p, \Phi_{2}\left(\sigma_{2}\left(u_{l}\right)\right)\right)$ is a local diffeomorphism from a neighborhood of $\left(\rho_{1}, 0\right)$ to a neighborhood of $u_{m}$. These neighborhoods can be made independent of $\left(u_{l}, u_{m}\right)$, for $\left(u_{l}, u_{m}\right)$ close enough to the original shock $\left(\mathfrak{u}_{1}, \bar{u}_{1}\right)$.

Moreover, $\sigma_{2}^{\prime}$ is negative, because, thanks to Lemma 1. up to lower order terms, we have

$$
\sigma_{2}=\sigma_{2}^{\prime} \frac{\lambda_{2}\left(u_{l}\right)-s}{\lambda_{2}\left(u_{m}\right)-s} \cdot \frac{\operatorname{det}\left(r_{2}\left(u_{l}\right), u_{m}-u_{l}\right)}{\operatorname{det}\left(r_{2}\left(u_{m}\right), u_{m}-u_{l}\right)},
$$

where $s$ is the speed of the strong shock. Using 25$), u_{m}-u_{l}=\left(\rho_{m}-\rho_{l}\right)^{t}(1, s)$ and Lax's inequalities, we see that the coefficient on the right hand side is positive. Hence $\sigma_{2}^{\prime}<0$, at least if the neighborhoods are small enough.

Interaction of a strong shock and a rarefaction front of opposite families. We consider a backward interaction of type strong 1-shock/2-rarefaction. The case of a backward interaction of type 1-rarefaction/strong 2-shock is treated similarly.

Lemma 8. Consider three states $u_{l}, u_{m}$ and $u_{r}$, where $u_{l}$ belongs to a neighborhood of $\mathfrak{u}_{1}^{-}$, while $u_{m}$ and $u_{r}$ are in a neighborhood of $\bar{u}_{1}$. Suppose they satisfy

$$
u_{r}=\Phi_{2}\left(\sigma_{2}, \Phi_{1}\left(\rho_{1}, u_{l}\right)\right), \quad u_{m}=\Phi_{1}\left(\rho_{1}, u_{l}\right),
$$

with $\rho_{1}<0, \sigma_{2}>0, \rho_{1}$ in a neighborhood of $\mu_{1}$, and $\sigma_{2}$ a neighborhood of 0 . Then one can find $\rho_{1}^{\prime}<0$ and $\sigma_{\gamma}<0$ such that

$$
\begin{gathered}
u_{r}=\Phi_{1}\left(\rho_{1}^{\prime}, \Phi_{1}\left(\sigma_{\gamma}, u_{l}\right)\right), \\
\left|\rho_{1}^{\prime}-\rho_{1}\right|+\left|\sigma_{\gamma}\right| \leq C_{3}\left|\sigma_{2}\right| .
\end{gathered}
$$

Remark 6. This interaction is hence solved quite differently from the previous one: one solves an interaction of type rarefaction/strong shock by two shocks of the same family (one strong, one weak).

Proof. Again, this is a consequence of the proof of Lemma 1 and the local inversion theorem. Indeed, given a shock $\left(u_{l}, u_{m}\right)$ close to $\left(\mathfrak{u}_{1}, \bar{u}_{1}\right), u_{m}=\Phi_{1}\left(\rho_{1}, u_{l}\right)$, we consider the map

$$
(F, G):\left(\gamma_{1}, \delta_{1}\right) \mapsto\left(\varepsilon_{1}, \varepsilon_{2}\right),
$$

where $\left(\rho_{1}+\varepsilon_{1}, \varepsilon_{2}\right)$ are the strengths of the waves in the Riemann problem corresponding to the states $\left(u_{l}, \Phi_{1}\left(\gamma_{1}, \Phi_{1}\left(\delta_{1}+\rho_{1}, u_{l}\right)\right)\right)$. Clearly,

$$
\partial_{2} G(0,0)=0 \quad \text { and } \quad \partial_{2} F(0,0)=1 .
$$

On the other hand, using Lemma 1 , we see that

$$
\partial_{1} G(0,0)=\frac{\lambda_{1}\left(u_{l}\right)-s}{\lambda_{2}\left(u_{m}\right)-s} \cdot \frac{\operatorname{det}\left(r_{1}\left(u_{l}\right), u_{m}-u_{l}\right)}{\operatorname{det}\left(r_{2}\left(u_{m}\right), u_{m}-u_{l}\right)} .
$$

Again using Lax's inequalities, one sees that the above coefficient is negative, which allows one to conclude by the local inversion theorem. 


\section{Interaction of the strong shocks}

Lemma 9. Consider three states $u_{l}, u_{m}$ and $u_{r}$ belonging respectively to some neighborhoods of $\mathfrak{u}_{1}^{-}, \tilde{u}_{1}$ and $\mathfrak{u}_{1}^{+}$, and satisfying

$$
u_{r}=\Phi_{2}\left(\rho_{2}, \Phi_{1}\left(\rho_{1}, u_{l}\right)\right), \quad u_{m}=\Phi_{1}\left(\rho_{1}, u_{l}\right),
$$

with $\rho_{1}$ and $\rho_{2}$ in some neighborhoods of $\mu_{1}, \mu_{2}$, respectively. Then one can find $\rho_{1}^{\prime}<0$ and $\rho_{2}^{\prime}<0$ such that

$$
u_{r}=\Phi_{1}\left(\rho_{1}^{\prime}, \Phi_{2}\left(\rho_{2}^{\prime}, u_{l}\right)\right)
$$

with

$$
\left|\left(\rho, \rho^{\prime}\right)-\left(\rho_{1}, \rho_{2}\right)\right| \leq \kappa\left|\left(u_{l}, u_{r}\right)-\left(\mathfrak{u}_{1}^{-}, \mathfrak{u}_{1}^{+}\right)\right| .
$$

Proof. We introduce the following map defined on $\mathcal{V}_{1}^{-} \times \mathcal{V}_{1}^{+} \times \mathcal{R}_{1} \times \mathcal{R}_{2}$, where $\mathcal{V}_{1}^{-}, \mathcal{V}_{1}^{+}$, $\mathcal{R}_{1}$ and $\mathcal{R}_{2}$ are respectively some neighborhoods of $\mathfrak{u}_{1}^{-}, \mathfrak{u}_{1}^{+}, \rho_{1}$ and $\rho_{2}$. By the implicit function theorem the map

$$
\left(u_{l}, u_{r}, \rho, \rho^{\prime}\right) \in \mathcal{V}_{1}^{-} \times \mathcal{V}_{1}^{+} \times \mathcal{R}_{1} \times \mathcal{R}_{2} \mapsto \Phi_{2}\left(\rho^{\prime}, u_{l}\right)-\Phi_{1}^{l}\left(\rho, u_{r}\right)
$$

has a zero at some $\left(\rho, \rho^{\prime}\right)$ for any $\left(u_{l}, u_{r}\right)$ in the neighborhood of $\left(\mathfrak{u}_{1}, \mathfrak{u}_{1}^{+}\right)$, provided the two curves $\Phi_{2}\left(\cdot, \mathfrak{u}_{1}^{-}\right)$and $\Phi_{1}^{l}\left(\cdot, \mathfrak{u}_{1}^{+}\right)$are transversal at the point $\tilde{u}_{1}$. This follows from the fact that these curves are respectively strictly convex and strictly concave, and meet at the points $(0,0)$ and $\tilde{u}_{1}$. Moreover, we get the estimate 95 .

\subsection{A domain for states in the solution}

Now we introduce $r>0$ such that:

- Lemma 6 applies in $B\left(\bar{u}_{1} ; r\right), B\left(U_{1}^{-} ; r\right), B\left(U_{1}^{+} ; r\right)$, and in $B\left(\tilde{u}_{1}^{-} ; r\right)$. This fixes the constant $C_{1}$ in (90).

- Lemmas 7 and 8 apply for $\left(u_{l}, u_{m}, u_{r}\right)$ belonging to $B\left(\bar{u}_{1} ; r\right) \times B\left(\bar{u}_{1} ; r\right) \times B\left(\mathfrak{u}_{1}^{+} ; r\right)$ or $B\left(\mathfrak{u}_{1}^{-} ; r\right) \times B\left(\bar{u}_{1} ; r\right) \times B\left(\bar{u}_{1} ; r\right)$. Lemma 7 also applies in $B\left(\tilde{u}_{1} ; r\right) \times B\left(\tilde{u}_{1} ; r\right) \times B\left(\mathfrak{u}_{1}^{+} ; r\right)$ or $B\left(\mathfrak{u}_{1}^{-} ; r\right) \times B\left(\tilde{u}_{1} ; r\right) \times B\left(\tilde{u}_{1} ; r\right)$. This fixes the constants $C_{2}$ in 92 and $C_{3}$ in 94$)$. (Of course, some of the conditions above are to be considered only when the left 1 shock (resp. the right 2-shock) is retained as described in Subsection 5.2 )

- Lemma 9 applies when $u_{l}, u_{m}$ and $u_{r}$ belong respectively to $B\left(\mathfrak{u}_{1}^{-} ; r\right), B\left(\bar{u}_{1} ; r\right)$ and $B\left(\mathfrak{u}_{1}^{+} ; r\right)$. (This is only useful in the case $\bar{u}_{1} \in \mathcal{D}_{2}$.)

- $B\left(U_{1}^{+} ; r\right)$ in the case $\bar{u}_{1} \in \mathcal{D}_{1} \cup \mathcal{C}_{1}$, or $B\left(\tilde{u}_{1} ; r\right)$ in the case $\bar{u}_{1} \in \mathcal{D}_{2}$, or $B\left(U_{1}^{-} ; r\right)$ in the case $\bar{u}_{1} \in \mathcal{D}_{3} \cup \mathcal{C}_{2}$, do not intersect the critical curves $\mathcal{C}_{1}, \mathcal{C}_{2}$.

- All characteristic speeds in $B\left(\bar{u}_{1} ; r\right)$ differ from $\bar{\lambda}_{1}$ and $\bar{\lambda}_{2}$ by at most $\beta$ and do not overlap.

- The shock joining a point in $B\left(\mathfrak{u}_{1}^{-} ; r\right)$ to a point in $B\left(\bar{u}_{1} ; r\right)$ have a speed that differs from the one of the original shock $\left(\mathfrak{u}_{1}^{-}, \bar{u}_{1}\right)$ by at most $\beta$, and similarly for the other strong shock.

Other conditions will arise in Section 5.8 . 
In the following we construct front-tracking approximations of a solution; all these approximations are piecewise constant solutions, in which all constant states belong to the domain

$$
\mathfrak{D}=B\left(\bar{u}_{1} ; r\right) \cup B\left(\mathfrak{u}_{1}^{-} ; r\right) \cup B\left(\mathfrak{u}_{1}^{+} ; r\right) \cup B\left(\tilde{u}_{1} ; r\right)
$$

\subsection{Approximations of the final state}

Now we describe the process that we use to approximate the final state, because here, in contrast to what is done in the usual front-tracking algorithm, the shape of the approximation is rather important. This is done by means of the following lemma.

Lemma 10. There are $\varepsilon_{1}>0$ and $C_{0}>0$ such that, if $T V\left(u_{1}\right)<\varepsilon_{1}$, then the function $U_{f} \in B V(\mathbb{R} ; \mathfrak{D})$ defined by $(88)$ can be approximated in the following way: there exists a sequence $\left(U_{f}^{n}\right)_{n \in \mathbb{N}^{*}}$ of functions on $[-1,2]$ such that:

$U_{f}^{n}$ is a piecewise constant function, constant in $[-1,0)$ and in $[1,2]$,

$$
\begin{gathered}
T V\left(U_{f}^{n}\right) \leq C_{0} T V\left(U_{f}\right), \\
U_{f}^{n} \rightarrow U_{f} \quad \text { in } L^{1}([-1,2]),
\end{gathered}
$$

and such that, for any point of discontinuity $A$ of $U_{f}^{n}$ in $(0,1)$, one has:

- Either $w^{1}\left(U_{f}^{n}\right)$ and $w^{2}\left(U_{f}^{n}\right)$ are both nondecreasing at A and $U_{f}^{n}$ satisfies

$$
U_{f}^{n}\left(A^{+}\right)=\hat{\Phi}_{1}\left(\delta_{1} / n^{2}, \hat{\Phi}_{2}\left(\delta_{2} / n^{2}, U_{f}^{n}\left(A^{-}\right)\right)\right) \quad \text { with } \delta_{1}, \delta_{2} \in\{0,1\}
$$

Moreover, if $w^{2}\left(U_{f}^{n}\right)\left(\right.$ resp. $\left.w^{1}\left(U_{f}^{n}\right)\right)$ increases at two distinct points of discontinuity $X$ and $Y$, then

$$
\begin{aligned}
& |X-Y| \geq \frac{1}{1-\alpha} \frac{\bar{\xi}_{1}}{n^{2}} \max \left(\frac{\bar{\lambda}_{2}-\bar{\lambda}_{1}}{1-Y}, \frac{\bar{\lambda}_{1}}{X}, \frac{\overline{-\lambda_{1}}}{1-Y}\right)^{-1}, \\
& \left(\operatorname{resp} .|X-Y| \geq \frac{1}{1-\alpha} \frac{\bar{\xi}_{2}}{n^{2}} \max \left(\frac{\bar{\lambda}_{2}-\bar{\lambda}_{1}}{X}, \frac{-\bar{\lambda}_{2}}{1-Y}, \frac{\bar{\lambda}_{2}}{X},\right)^{-1}\right) .
\end{aligned}
$$

- Or $w^{1}\left(U_{f}^{n}\right)$ and $w^{2}\left(U_{f}^{n}\right)$ are both nonincreasing at $A$ and $U_{f}^{n}$ satisfies

$$
U_{f}^{n}\left(A^{+}\right)=\Psi_{1}^{n}\left(q_{1} / n, \Psi_{2}^{n}\left(q_{2} / n, U_{f}^{n}\left(A^{-}\right)\right)\right) \quad \text { for some } q_{1}, q_{2} \in \mathbb{Z}^{-} \cup\{0\} .
$$

Finally, at the points of discontinuity 0 and $1, U_{f}^{n}$ satisfies:

$$
\begin{aligned}
& U_{f}^{n}\left(0^{+}\right)=\Phi_{1}\left(\mu_{1}, U_{f}^{n}\left(0^{-}\right)\right), \\
& U_{f}^{n}\left(1^{+}\right)=\Phi_{2}\left(\mu_{2}, U_{f}^{n}\left(1^{-}\right)\right) .
\end{aligned}
$$


The idea of this lemma is to show that one can approximate the final state by piecewise constant states, in which discontinuities can be reached either by "shock fans" with a fixed strength for each shock, or by rarefaction fronts of a fixed size, which is of lower order.

Proof of Lemma 10. We consider $U_{f}$ as in (88). Without loss of generality, we can assume that $U_{f}$ is right-continuous. We first introduce the functions

$$
\hat{W}_{1}: x \mapsto w^{1}\left(U_{f}(x)\right) \quad \text { and } \quad \hat{W}_{2}: x \mapsto w^{2}\left(U_{f}(x)\right), \quad \text { for } x \in[-1,2] .
$$

These functions are of bounded variation and hence can be decomposed into increasing and decreasing parts, say

$$
\hat{W}_{1}=\bar{W}_{1}+\underline{W}_{1}, \quad \hat{W}_{2}=\bar{W}_{2}+\underline{W}_{2},
$$

where $\bar{W}_{1}$ and $\bar{W}_{2}$ (resp. $\underline{W}_{1}, \underline{W}_{2}$ ) are nondecreasing (resp. nonincreasing). We introduce the functions

$$
\underline{W}=\left(\begin{array}{l}
\underline{W}_{1} \\
\underline{W}_{2}
\end{array}\right), \quad \bar{W}=\left(\begin{array}{l}
\bar{W}_{1} \\
\bar{W}_{2}
\end{array}\right) .
$$

We fix $n \in \mathbb{N}^{*}$ and $\varepsilon>0$. As follows from $\sqrt{79}-\sqrt{80}$, the function $\bar{W}$ is Lipschitz outside 0 and 1 (note that at 0 and 1 , the function $U_{f}$ is decreasing in both coordinates, hence $\bar{W}$ is continuous). Hence we can approximate $\bar{W}$ with accuracy $\varepsilon$ in $L^{\infty}$ norm by rightcontinuous piecewise constant functions, in which each jump is of amplitude 0 or $1 / n^{2}$ (for each coordinate), and which satisfy the following constraints:

$$
\begin{gathered}
\left\|\bar{W}^{n}-\bar{W}\right\|_{L^{\infty}([-1,2])} \leq \varepsilon, \\
T V\left(\bar{W}^{n}\right) \leq T V(\bar{W}),
\end{gathered}
$$

and moreover we require that $\bar{W}^{n}$ is constant on both $[-1,0)$ and $[1,2]$, and continuous at 0 and 1 . Finally, we require that for some points $x_{0}, \ldots, x_{m+1}$, one has

$$
\bar{W}^{n}\left(x_{i}\right)=\bar{W}\left(x_{i}\right),
$$

hence two discontinuity points of $\bar{W}^{n}$ satisfy 102 .

We let $I_{0}, \ldots, I_{m+1}$ be the jumps of $\bar{W}^{n}$ in increasing order, with $I_{0}>0$ and $I_{m+1}<1$.

Now we approximate $\underline{W}$ with accuracy $\varepsilon$ by a piecewise constant function $\underline{W}^{n}$, which is also required to be decreasing in both coordinates:

$$
\begin{gathered}
\left\|\underline{W}^{n}-\underline{W}\right\|_{L^{1}([-1,2])} \leq \varepsilon, \\
T V\left(\underline{W}^{n}\right) \leq T V(\underline{W}) .
\end{gathered}
$$

Again, we require that $W^{n}$ is constant on both $[-1,0)$ and [1, 2]. Moreover, we ask that $\underline{W}^{n}\left(0^{ \pm}\right)=\underline{W}\left(0^{ \pm}\right)$and $\underline{W}^{n}\left(1^{ \pm}\right)=\underline{W}\left(1^{ \pm}\right)$. Call its discontinuity points $D_{0}, \ldots, D_{l+1}$ with $D_{0}=0$ and $D_{l+1}=1$.

First, we slightly modify the discontinuity points in $\underline{W}^{n}$ in such a way that all points $D_{i}$ and $I_{j}$ are distinct. This can be done by moving each point $D_{i}$ that coincides with a point $I_{j}$ a small distance. This adds an error $\varepsilon$ in $(110)$, but does not affect (111). 
Then we modify the values of $\underline{W}^{n}$ in order that $U_{n}^{f}$ fits the requirements, from left to right (that is, starting from -1 ), keeping the same discontinuity points. The modified function will be denoted $\underline{\tilde{W}}^{n}$. Let us recall that in this subsection, all functions are rightcontinuous. We describe the modifications recursively:

- We do not modify $\underline{W}^{n}$ on $\left[-1, D_{0}\right]$ and on $\left[D_{0}, D_{1}\right]$.

- If we have already modified $\underline{W}^{n}$ on $\left[D_{i}, D_{i+1}\right.$ ) for all $i=0, \ldots, k-1$ (with $k \leq l$ ) we change the value of $\underline{W}^{n}$ in $\left[\bar{D}_{k}, D_{k+1}\right)$ in the following way. For some $w, z$, we have

$$
\left[\bar{W}^{n}+\underline{W}^{n}\right]\left(D_{k}\right)=\Psi_{1}^{n}\left(w, \Psi_{2}^{n}\left(z,\left[\bar{W}^{n}+\underline{\tilde{W}}^{n}\right]\left(D_{k-1}\right)\right)\right) \text {. }
$$

We then define $\underline{\tilde{W}}^{n}\left(D_{k}\right)$ so that it satisfies

$$
\left[\bar{W}^{n}+\underline{\tilde{W}}^{n}\right]\left(D_{k}\right)=\Psi_{1}^{n}\left(\lceil n w\rceil_{-} / n, \Psi_{2}^{n}\left(\lceil n z\rceil_{-} / n,\left[\bar{W}^{n}+\underline{\tilde{W}}^{n}\right]\left(D_{k-1}\right)\right)\right) .
$$

- At the point $D_{l+1}=1$, define $\underline{\tilde{W}}^{n}\left(D_{l+1}\right)$ by

$$
\left[\bar{W}^{n}+\underline{\tilde{W}}^{n}\right]\left(D_{l+1}\right)=\Phi_{2}^{n}\left(\mu_{2},\left[\bar{W}^{n}+\underline{\tilde{W}}^{n}\right]\left(D_{l}\right)\right) .
$$

Now we fix

$$
U_{f}^{n}:=\bar{W}^{n}+\underline{\tilde{W}}^{n} \quad \text { in Riemann coordinates. }
$$

Properties (98), 103, 104 and (105) are direct consequences of the construction. Property (102) is a consequence of (109). It remains to check (99) and (100).

We remark that during the construction, we have not modified the total variation of $\underline{W}_{1}$ and $\underline{W}_{2}$, except perhaps at $x=1$ where the modification is clearly of the same order as $\mu_{2}$, which leads to 99 .

Concerning (100), let us show that for $k \leq l$ and $i=1,2$,

$$
\underline{W}_{i}^{n}\left(D_{k}\right)-\underline{\tilde{W}}_{i}^{n}\left(D_{k}\right) \leq C \frac{T V_{\left[D_{0}, D_{k}\right]}\left(\underline{W}^{n}\right)}{n^{2}} .
$$

This is clear for $k=0$. Now suppose we have proven 116 for $k<l$, and let us prove it for $k+1$. Let $w$ and $z$ be as in (112). There are various situations:

- $w \leq 0$ and $z \leq 0$ : then using the monotonicity of $\Psi_{i}^{n}$ and 113 , we see that $(116)$ is satisfied because the left hand side is nonpositive.

- $w \leq 0$ and $z>0$ : then again $(116)$ is satisfied for $i=2$ because the left hand side is nonpositive for $i=2$. For $i=1$, this is a consequence of the fact that $\underline{W}^{n}$ is nonincreasing, of (37) and of the induction hypothesis.

- $w \leq 0$ and $z>0$ : this is done as in the previous case.

- $w>0$ and $z>0$ : this could be treated as above, but in fact this case does not occur: if it did, then using the monotonicity of $\Psi_{i}^{n}$ one would have for $i=1,2, \underline{\underline{W}}_{i}^{n}\left(D_{k-1}\right)<$ $\underline{W}_{i}^{n}\left(D_{k}\right) \leq \underline{W}_{i}^{n}\left(D_{k-1}\right)$. Using 113 , one sees that we were in the same situation at the point $D_{k-1}$, which yields a contradiction. 
Now when $\underline{W}_{i}^{n}\left(D_{k}\right)<\underline{\tilde{W}}_{i}^{n}\left(D_{k}\right)$, it follows from the construction that $\underline{\tilde{W}}_{i}^{n}\left(D_{k}\right)-\underline{W}_{i}^{n}\left(D_{k}\right)$ $\leq 1 / n$. Hence

$$
\left\|U_{n}^{f}-U_{f}\right\|_{L^{1}([-1,2])} \leq\left\|\hat{U}-\underline{W}^{n}-\bar{W}^{n}\right\|_{L^{1}([-1,2])}+\left\|\underline{W}^{n}-\underline{\tilde{W}}^{n}\right\|_{L^{1}([-1,2])} \rightarrow 0 .
$$

In what follows, we write $\underline{W}^{n}$ for $\underline{\tilde{W}}^{n}$ for notational convenience.

\subsection{Front-tracking approximations}

Now, "starting" from $U_{f}^{n}$, we construct (backward in time) a piecewise constant approximate solution $U^{n}$ of the problem.

Step 1. Given the approximation $U_{f}^{n}$ of the final state, we begin by solving approximately the Riemann problems backward in time:

At a point of discontinuity of $\underline{W}^{n}$ (except 0 and 1 ), we approximate the solution by shock fans. Let $A$ be such a point of discontinuity. By Lemma 10 , at such points, one has

$$
U_{f}^{n}\left(A^{+}\right)=\Psi_{1}^{n}\left(k_{1} / n, \Psi_{2}^{n}\left(k_{2} / n, U_{f}^{n}\left(A^{-}\right)\right)\right)
$$

for some nonpositive integers $k_{1}$ and $k_{2}$. Then we introduce the following intermediate states:

$$
\begin{array}{rlrl}
\omega_{0} & =U_{f}^{n}\left(A^{-}\right), & \\
\omega_{i} & =\Psi_{2}^{n}\left(i / n, U_{f}^{n}\left(A^{-}\right)\right) & & \text {for } 0 \leq i \leq k_{2}, \\
\omega_{i} & =\Psi_{1}^{n}\left(\left(i-k_{2}\right) / n, \Psi_{2}^{n}\left(k_{2} / n, U_{f}^{n}\left(A^{-}\right)\right)\right) & & \text {for } k_{2}+1 \leq i \leq k_{1}+k_{2}, \\
\omega_{k_{1}+k_{2}} & =U_{f}^{n}\left(A^{+}\right) . & &
\end{array}
$$

We also introduce the following front lines:

$$
x_{i}(t)=A+\lambda_{i} t \quad \text { for } t \leq 0, \quad \text { where } \quad \lambda_{i}=s\left(\omega_{i}, \omega_{i+1}\right) \quad \text { for } i=0, \ldots, k_{1}+k_{2}-1 .
$$

Then locally, the backward Riemann problem is approximately solved by

$$
u(t, x)= \begin{cases}U_{f}^{n}\left(A^{-}\right) & \text {for } x<x_{0}(t), \\ \omega_{i} & \text { for } x_{i}(t)<x<x_{i+1}(t), \\ U_{f}^{n}\left(A^{+}\right) & \text {for } x>x_{k_{1}+k_{2}-1}(t) .\end{cases}
$$

(Note that $x_{i}<x_{i+1}$ by Lax's inequalities.)

At the points 0 and 1 which are discontinuity points for $\underline{W}^{n}$, we solve the backward Riemann problem by a single strong 2 -shock (resp. 1-shock) (at exact shock speed).

At a point of discontinuity of $\bar{W}^{n}$, we approximate the solution by (single) rarefaction fronts. Let $A$ be such a point of discontinuity. Then by construction one has

$$
U_{f}^{n}\left(A^{+}\right)=\hat{\Phi}_{1}\left(\delta_{1} / n^{2}, \hat{\Phi}_{2}\left(\delta_{2} / n^{2}, U_{f}^{n}\left(A^{-}\right)\right)\right)
$$


where $\delta_{i}=0$ or 1 . We define

$$
\begin{aligned}
& \omega_{0}=U_{f}^{n}\left(A^{-}\right), \\
& \omega_{1}=\hat{\Phi}_{2}\left(\delta_{2} / n^{2}, U_{f}^{n}\left(A^{-}\right)\right), \\
& \omega_{2}=\hat{\Phi}_{1}\left(\delta_{1} / n^{2}, \hat{\Phi}_{2}\left(\delta_{2} / n^{2}, U_{f}^{n}\left(A^{-}\right)\right)\right)=U_{f}^{n}\left(A^{+}\right) .
\end{aligned}
$$

We also introduce the following front lines:

$$
x_{i}(t)=A+\lambda_{i} t \quad \text { for } t \leq 0, \quad \text { where } \quad \lambda_{i}=s\left(\omega_{i}, \omega_{i+1}\right) \quad \text { for } i=0,1 .
$$

Then locally, the backward Riemann problem is approximately solved by

$$
u(t, x)= \begin{cases}U_{f}^{n}\left(A^{-}\right) & \text {for } x<x_{0}(t), \\ \omega_{1} & \text { for } x_{0}(t)<x<x_{1}(t), \\ U_{f}^{n}\left(A^{+}\right) & \text {for } x>x_{1}(t) .\end{cases}
$$

Of course, we suppress unnecessary lines (i.e. $x_{0}$ if $\omega_{0}=\omega_{1}$ or $x_{1}$ if $\omega_{1}=\omega_{2}$ ). Note that $x_{0}<x_{1}$ because the first and second characteristic speeds do not overlap in the domain.

This defines the approximation for small $t \leq 0$ as long as two fronts do not meet. Note that we do not modify the speeds of the front as in Section 3 . In fact, we will prove that fronts of the same family do not meet at least if $T V\left(u_{1}\right)$ is small enough, and $n$ is large enough. As a consequence, in these circumstances, without modifying the speeds at all, there are only binary interactions. Possibly, there can be simultaneous interactions in different parts of the domains, but these can be treated as successive interactions.

Marking. We call the 1-shock issuing from $x=0$ and the 2-shock issuing from $x=1$ strong. All other waves are weak. The $i$-waves across which $w^{3-i}$ increases are rarefaction fronts, whereas the ones for which $w^{3-i}$ decreases are shocks.

Step 2. We have to explain how to extend the solution after two fronts have met. We discuss the way to extend it according to the nature of the incoming fronts.

\section{Fronts of the same family.}

Weak shocks. Two shocks of the same family cannot meet when going backward in time, as a direct consequence of Lax's inequalities.

Rarefaction-rarefaction. This is precisely the kind of interaction that we want to avoid. We will prove that such meetings do not take place inside the domain. Let us say for the moment that the two fronts merge in the following sense: say the front on the left separates the states $\omega_{l}$ and $\omega_{m}$, and the right one separates $\omega_{m}$ from $\omega_{r}$. "After" the meeting, we define the approximate solution by a single discontinuity line separating $\omega_{l}$ from $\omega_{r}$ and traveling at speed $s\left(\omega_{l}, \omega_{r}\right)$.

Shock-rarefaction. Again, we will show that these interactions do not take place, as a consequence of the fact that the strength of the shocks is greater than the one of the rarefaction fronts. Let us temporarily say that these interactions are solved as in the previous case. 


\section{Fronts of opposite families.}

Weak fronts. When two weak fronts $\left(u_{l} ; u_{m}\right)$ with $u_{m}=\Phi_{1}\left(\sigma_{1}, u_{l}\right)$ and $\left(u_{m} ; u_{r}\right)$ with $u_{r}=\Phi_{2}\left(\sigma_{2}, u_{m}\right)$ interact, we introduce $\sigma_{1}^{\prime}$ and $\sigma_{2}^{\prime}$ by Lemma 6 and $\tilde{u}_{m}:=\Phi_{2}\left(\sigma_{2}^{\prime}, u_{l}\right)$. After the interaction, we extend the approximation by two fronts separating $u_{l}$ and $\tilde{u}_{m}$ on the left, and separating $\tilde{u}_{m}$ and $u_{r}$ on the right, with respective speeds $s\left(u_{l}, \tilde{u}_{m}\right)$ and $s\left(\tilde{u}_{m}, u_{r}\right)$. Note that at least for small fronts, the outgoing $i$-wave has the same nature (shock/rarefaction) as the incoming one, as a consequence of $(90)$ for instance.

Strong shocks. The interaction of the two strong shocks is solved in terms of two (strong) shocks, as described in Lemma 9 that is, if the (left) 1 -shock is described by $u_{m}=$ $\Phi_{1}\left(\rho_{1}, u_{l}\right)$, while the (right) 2 -shock is described by $u_{r}=\Phi_{2}\left(\rho_{2}, u_{m}\right)$, we find $\rho_{1}^{\prime}$ and $\rho_{2}^{\prime}$ by Lemma 9 As in the previous case, we extend the solution by a 2 -shock separating $u_{l}$ from $\tilde{u}_{m}:=\Phi_{2}\left(\rho_{2}^{\prime}, u_{l}\right)$ with speed $s\left(u_{l}, \tilde{u}_{m}\right)$ and a 1-shock separating $\tilde{u}_{m}$ from $u_{r}$ with speed $s\left(\tilde{u}_{m}, u_{r}\right)$.

Strong shock-weak shock. The interaction of a weak 1-shock $\left(u_{l}, u_{m}\right)$ and a strong 2shock $\left(u_{m}, u_{r}\right)$ is solved as in the weak/weak case, with $\rho_{1}^{\prime}$ and $\sigma_{2}^{\prime}$ given by Lemma 7 This case of a strong 1 -shock and a weak 2 -shock is handled similarly.

Strong shock-rarefaction. The interaction of the 1-strong shock $\left(u_{l}, u_{m}\right)$ and a 2-rarefaction front $\left(u_{m}, u_{r}\right)$ is solved in terms of two 1-shocks as made possible by Lemma 8 we extend the solution by a 1 -shock separating $u_{l}$ from $\tilde{u}_{m}:=\Phi_{1}\left(\sigma_{\gamma}, u_{l}\right)$ with speed $s\left(u_{l}, \tilde{u}_{m}\right)$ and a strong 1 -shock separating $\tilde{u}_{m}$ from $u_{r}$ with speed $s\left(\tilde{u}_{m}, u_{r}\right)$. The case of an interaction strong 2-shock/1-rarefaction front is treated similarly.

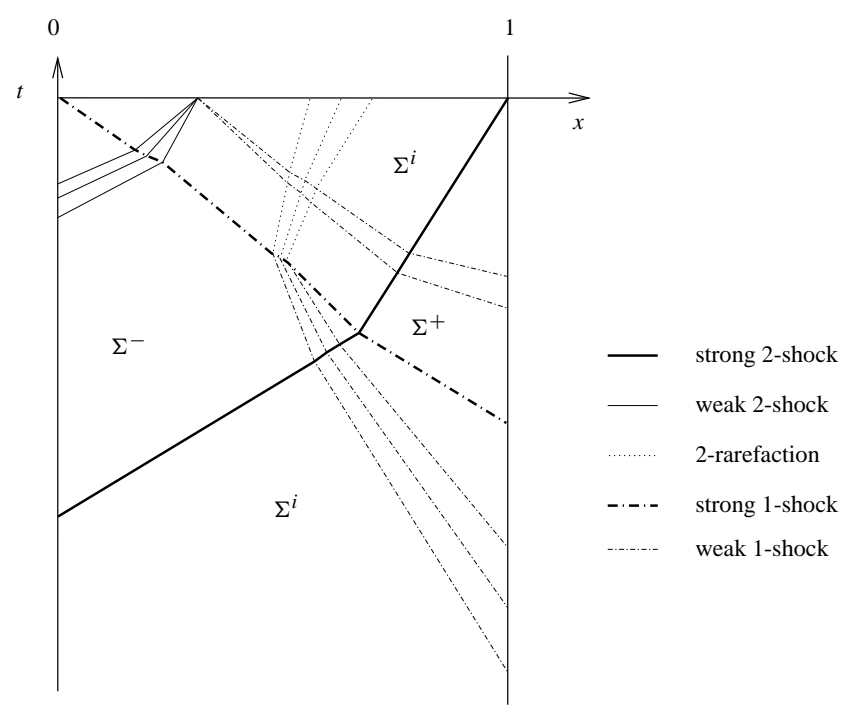

Fig. 4. A backward front-tracking approximation. 
Marking. In all cases but the case of an interaction between a strong shock and a rarefaction front, the $i$-outgoing wave is called weak (resp. strong) if the incoming wave is weak (resp. strong). Concerning the case of a strong 1-shock meeting with a 2-rarefaction front, the outgoing 1-shock on the left is called weak and the 1-shock on the right is strong. Correspondingly, in the case of a strong 2-shock meeting with a 1-rarefaction front, the outgoing 2-shock on the right is called weak and the 2-shock on the left is strong.

Remark 7. In the previous algorithm, two incoming fronts yield at most two outgoing fronts, hence the total number of fronts is finite.

The construction described above is represented in Figure 4

\subsection{Estimates on the approximation}

In this section, we establish some estimates on the approximation described above.

Step 1. BV estimate. As in Section 3, we introduce the following Glimm's interaction functionals, with different weights according to the nature of the waves:

$$
\begin{aligned}
& Q_{w w}(t)=\sum_{\substack{(\alpha, \beta) \text { approaching } \\
\text { weak waves }}}\left|\sigma_{\alpha}\right|\left|\sigma_{\beta}\right|, \\
& Q_{s w S}(t)=\sum_{\substack{(\alpha, \beta) \text { approaching with } \\
\alpha \text { a weak shock } \\
\beta \text { a strong wave }}}\left|\sigma_{\alpha}\right|, \\
& Q_{s w R}(t)=\sum_{\substack{(\alpha, \beta) \text { approaching with } \\
\alpha \text { a weak rarefaction } \\
\beta \text { a strong wave }}}\left|\sigma_{\alpha}\right|,
\end{aligned}
$$

where approaching fronts are couples $(\alpha, \beta)$ such that $\alpha$ is a 2-front on the right of the 1front $\beta$ and the strength is measured for instance by (36). The total interaction functionals are defined as

$$
Q(t)=\mathcal{A} Q_{w w}(t)+Q_{s w S}(t)+\mathcal{B} Q_{s w R}(t),
$$

where $\mathcal{A}$ and $\mathcal{B}$ are constants to be fixed later.

We also introduce various strengths of the approximation at time $t: V_{s}^{1}(t)\left(\right.$ resp. $\left.V_{s}^{2}(t)\right)$ is the strength of the strong 1-shock (resp. 2-shock) at time $t$ and

$$
V_{w}(t)=\sum_{\substack{\alpha \text { weak wave existing } \\ \text { at time } t}}\left|\sigma_{\alpha}\right|, \quad V_{s}(t)=V_{s}^{1}(t)+V_{s}^{2}(t), \quad V(t)=V_{w}(t)+V_{s}(t)
$$

During an interaction:

$$
\left(\sigma_{\alpha} \text { and } \sigma_{\beta} \text { at time } \tau^{+}\right) \rightarrow\left(\sigma_{\alpha}^{\prime} \text { and } \sigma_{\beta}^{\prime} \text { at time } \tau^{-}\right)
$$


we have the estimate 90 for a weak-weak interaction, 92 or 94) for a strong-weak interaction, 95 for a strong interaction (in the case where there are two strong shocks). Let $T_{I}$ be the time of interaction of the two strong waves (if any). All the previous estimates are valid as long as the states considered are in the domain $\mathfrak{D}$. As in Section 3 , we get:

- during a weak-weak interaction:

$$
\begin{aligned}
& V_{w}\left(\tau^{-}\right) \leq V_{w}\left(\tau^{+}\right)+C_{1}|\sigma|\left|\sigma^{\prime}\right|, \quad V_{s}\left(\tau^{-}\right)=V_{s}\left(\tau^{+}\right), \\
& Q_{w w}\left(\tau^{-}\right)-Q_{w w}\left(\tau^{+}\right) \leq-|\sigma|\left|\sigma^{\prime}\right|+C_{1}|\sigma|\left|\sigma^{\prime}\right| V_{w}\left(\tau^{+}\right), \\
& Q_{s w S}\left(\tau^{-}\right)-Q_{w s S}\left(\tau^{+}\right) \leq C_{1}|\sigma|\left|\sigma^{\prime}\right|, \quad Q_{s w R}\left(\tau^{-}\right)-Q_{w s R}\left(\tau^{+}\right) \leq C_{1}|\sigma|\left|\sigma^{\prime}\right|,
\end{aligned}
$$

- during a strong shock-weak shock interaction:

$$
\begin{aligned}
& V_{w}\left(\tau^{-}\right) \leq V_{w}\left(\tau^{+}\right)+C_{2}|\sigma|, \quad V_{s}\left(\tau^{-}\right) \leq V_{s}\left(\tau^{+}\right)+C_{2}|\sigma|, \\
& Q_{w w}\left(\tau^{-}\right)-Q_{w w}\left(\tau^{+}\right) \leq C_{2}|\sigma| V_{w}(\tau), \\
& Q_{s w S}\left(\tau^{-}\right)-Q_{s w S}\left(\tau^{+}\right)=-|\sigma|, \quad Q_{s w R}\left(\tau^{-}\right)-Q_{s w R}\left(\tau^{+}\right)=0,
\end{aligned}
$$

- during a strong shock-weak rarefaction interaction (call the rarefaction $\sigma$ )

$$
\begin{aligned}
& V_{w}\left(\tau^{-}\right) \leq V_{w}\left(\tau^{+}\right)+C_{3}|\sigma|, \quad V_{s}\left(\tau^{-}\right) \leq V_{s}\left(\tau^{+}\right)+C_{3}|\sigma|, \\
& Q_{w w}\left(\tau^{-}\right)-Q_{w w}\left(\tau^{+}\right) \leq C_{3}|\sigma| V_{w}\left(\tau^{-}\right), \\
& Q_{s w S}\left(\tau^{-}\right)-Q_{s w S}\left(\tau^{+}\right) \leq C_{3}|\sigma|, \quad Q_{s w R}\left(\tau^{-}\right)-Q_{s w R}\left(\tau^{+}\right)=-|\sigma| .
\end{aligned}
$$

Now we choose $\mathcal{A}$ and $\mathcal{B}$ :

$$
\mathcal{B}=4 C_{3} \quad \text { and } \quad \mathcal{A}=3 C_{1}(1+\mathcal{B}) .
$$

Hence for a suitable $c_{1}>0$ one has: for $\tau>T_{I}$, if $V_{w}\left(\tau^{+}\right)<c_{1}$,

$$
\begin{cases}Q\left(\tau^{-}\right)-Q\left(\tau^{+}\right) \leq-(\mathcal{A} / 2)|\sigma|\left|\sigma^{\prime}\right| & \text { in a weak-weak interaction, } \\ Q\left(\tau^{-}\right)-Q\left(\tau^{+}\right) \leq-(1 / 2)|\sigma| & \text { in a strong shock-weak shock interaction, } \\ Q\left(\tau^{-}\right)-Q\left(\tau^{+}\right) \leq-(\mathcal{B} / 2)|\sigma| & \text { in a strong shock-weak rarefaction interaction. }\end{cases}
$$

Hence there is some $K>0$ such that as long as $\tau>T_{I}$ and $V_{w}\left(\tau^{+}\right)<c_{1}$,

$\tau \mapsto V_{w}(\tau)+K Q(\tau)$ and $\tau \mapsto V_{S}(\tau)+K Q(\tau)$ are nonincreasing as $\tau$ decreases,

$\tau \mapsto V_{s}(\tau)-K Q(\tau)$ is nondecreasing as $\tau$ decreases.

Moreover, at each interaction time, the decrease (resp. increase) of the above quantities is at least $(K / 2) \Delta Q$.

It follows as in Section 3 that provided $V_{w}(0)$ is small enough, one has $V_{w}(\tau)<c_{1}$ and hence (125) satisfied for all $t$ in $\left[T_{I}, 0\right]$, at least as long as the approximation is welldefined.

For times $t \leq T_{I}$, the total strength can be estimated in the same way but the situation is much simpler, because there is no rarefaction front outside the triangular zone delimited 
by the initial interval $\{0\} \times[0,1]$ and the two strong shocks before their interaction (or outside the triangle determined by the strong shock until it quits the domain, when there is only one strong shock). One gets again

$$
\begin{aligned}
& \tau \mapsto V_{w}(\tau)+K Q(\tau) \text { is nonincreasing as } \tau \leq T_{I}^{-} \text {decreases, } \\
& \tau \mapsto V_{s}(\tau)+K Q(\tau) \text { is nonincreasing as } \tau \leq T_{I}^{-} \text {decreases, } \\
& \tau \mapsto V_{s}(\tau)-K Q(\tau) \text { is nondecreasing as } \tau \leq T_{I}^{-} \text {decreases, } \\
& V_{w}(\tau)=O(1) V_{w}(0) \forall \tau \leq T_{I} .
\end{aligned}
$$

(Note that $V_{w}$ and $Q_{w w}$ are continuous at time $T_{I}$.)

Step 2. Validity of the domain. We prove that if the total variation of $u_{1}$ is small enough, then all the states considered in the approximations lie in the domain $\mathfrak{D}$ introduced in Subsection 5.4 and the previous estimates are valid.

We denote by $\Sigma^{-}(\tau)$ (resp. $\left.\Sigma^{+}(\tau), \Sigma^{i}(\tau)\right)$ the zone in $\mathbb{R}$ of points on the left of the left strong shock (resp. on the right of the right strong shock, between the two strong shocks). If one of the two shocks is missing (i.e. $\bar{u}_{1} \notin \mathcal{D}_{2}$ according to Subsection 5.2), we agree that there is no $\Sigma^{i}(\tau)$ and that $\Sigma^{-}(\tau)$ (resp. $\left.\Sigma^{+}(\tau)\right)$ is the zone to the left (resp. right) of the unique strong shock.

Denote by $S_{1}$ (resp. $S_{2}$ ) the position of the strong 1-shock (resp. 2-shock) (see Figure 4).

First, it is quite easy to see that the states in $\Sigma^{-}(\tau)$ (resp. $\left.\Sigma^{+}(\tau)\right)$ stay close to $\mathfrak{u}_{1}^{-}$ (resp. $\mathfrak{u}_{1}^{+}$) if the total variation of $u_{1}$ has been chosen small enough. Indeed, we observe that

$$
V_{w}(\tau) \leq V_{w}(0)+K Q(0) \leq K_{2} T V\left(u_{1}\right) .
$$

Hence the total variation on these zones satisfies

$$
T V_{\Sigma^{-}(\tau) \cup \Sigma^{+}(\tau)}\left(U^{n}\right) \leq L K_{2} T V\left(u_{1}\right),
$$

with $L$ defined as in 65 .

As in the previous construction the leftmost (resp. rightmost) state is $U_{1}^{-}$(resp. $U_{n}^{f}(1)$ with $\left.\left|U_{n}^{f}(1)-U_{1}^{+}\right|=O(1 / n)\right)$, the claim follows, at least for $n$ large enough.

It remains to see that in $\Sigma^{i}(\tau)$, the states are close to $\bar{u}_{1}$ for $\tau \geq T_{I}$ and to $\tilde{u}_{1}$ for $\tau \leq T_{I}$. Concerning the first point, it follows from 125 that the strength of the strong shocks is as close to the original one as wanted if $T V\left(u_{1}\right)$ is small enough. As the left state is close to $\mathfrak{u}_{1}^{-}$, it follows that the state $U^{n}\left(\tau, S_{1}(\tau)^{+}\right)$is as close to $\bar{u}_{1}$ as required. The claim for $\tau \geq T_{I}$ follows as previously. All the same, the claim for $\tau \leq T_{I}$ follows from (95) and the same procedure.

\section{Step 3. Estimate on the size of the waves}

Rarefaction fronts. This is done essentially as the estimate of rarefaction fronts in Section 3 All the calculations in this step are valid as long as there is no interaction between fronts of one family. This will allow us to prove in the next step that such interactions are not possible, and hence that the estimates here are valid for all times. 
Let us first remark that all the interactions of two fronts in the algorithm are solved in terms of at most two outgoing fronts, and that one can track the front after interactions by considering the outgoing front of the same family.

We consider a rarefaction front $v$, and introduce $V_{v}$ as in (66) where we now say that $\mu$ and $\nu$ are approaching one another if they are in opposite families and the 2-wave is on the right of the 1 -wave. For a quantity $g$ depending on the approximation, define $\Delta g(\tau)=g\left(\tau^{-}\right)-g\left(\tau^{+}\right)$. Recalling that the meeting of $v$ with a strong shock ends the rarefaction front, the evolution of $V_{v}$ is ruled by weak interactions, for which we still have Glimm type estimates. Hence as in Section 3 (see 67), this leads to

$$
\left|\sigma_{v}(t)\right| \leq \frac{1}{n^{2}} \exp \left(C_{1}\left(V_{w}(0)+K Q(0)\right)\right)=O\left(1 / n^{2}\right) .
$$

Weak shocks before any strong interaction. We consider a weak shock front $\alpha$, and introduce $V_{\alpha}$ by 66 as well. We consider the evolution of the strength of the shock before any interaction with a strong wave. One has

- during an interaction which does not involve $\alpha$ :

$$
\Delta \sigma_{\alpha}=0 \quad \text { and } \quad \Delta\left(V_{\alpha}+K Q\right) \leq 0,
$$

- during an interaction which involves $\alpha$ and a weak wave (denoted $\beta$ ):

$$
\Delta \sigma_{\alpha} \leq C_{1}\left|\sigma_{\alpha}^{-}\right|\left|\sigma_{\beta}\right| \quad \text { and } \quad \Delta\left(V_{\alpha}+K Q\right) \leq-\left|\sigma_{\beta}\right|
$$

Now we introduce the function

$$
G(t):=\left|\sigma_{\alpha}(t)\right| \exp (g(t)) \quad \text { with } \quad g(t):=-C_{5}\left(V_{\alpha}(t)+K Q(t)\right),
$$

where we fix the constant $C_{5}:=4 C_{1}$. It is clear that in the first case, $G$ decreases with $t$ (that is, increases as $t$ decreases). For the second case, we remark that thanks to (125), if $T V\left(u_{1}\right)$ is small enough, one has for all times

$$
-1 \leq g(t) \leq 0
$$

Since $1-e^{x} \geq-(1-1 / e) x$ on $[-1,0]$, one gets, for each interaction time,

$$
\begin{aligned}
\Delta G(t) & =G\left(t^{+}\right)\left\{\Delta\left|\sigma_{\alpha}\right|+\left|\sigma_{\alpha}\right|\left[1-\exp \left(g\left(t^{+}\right)-g\left(t^{-}\right)\right)\right]\right\} \\
& \geq G\left(t^{+}\right)\left\{\Delta\left|\sigma_{\alpha}\right|-\left|\sigma_{\alpha}\right|(1-1 / e)\left(g\left(t^{+}\right)-g\left(t^{-}\right)\right)\right\} \\
& \geq G\left(t^{+}\right)\left\{-C_{1}\left|\sigma_{\alpha}\right|\left|\sigma_{\beta}\right|+\left|\sigma_{\alpha}\right|(1-1 / e)\left(C_{5}\left|\sigma_{\beta}\right|\right)\right\} \geq 0 .
\end{aligned}
$$

Hence $G$ decreases with $t$ (i.e. increases as $t \downarrow$ ), and hence we deduce

$$
\left|\sigma_{\alpha}(t)\right| \geq \frac{1}{n} \exp \left(-C_{5}\left(V_{w}(0)+K Q(0)\right)\right)
$$

Shocks after a strong interaction. Clearly, after a strong interaction, by (92), the size of the outgoing wave is connected to the size of the incoming wave via

$$
\left|\sigma\left(\tau_{i}^{-}\right)\right| \leq C_{2}\left|\sigma\left(\tau_{i}^{+}\right)\right|
$$


where $\tau_{i}$ is the time of interaction of the weak wave with a strong shock. Hence the same argument as previously leads to the conclusion that for all weak waves $\gamma$ one has, for $\tau \leq T_{I}$,

$$
\left|\sigma_{\gamma}(\tau)\right| \leq\left|\sigma_{\gamma}\left(\tau_{i}^{+}\right)\right| \exp \left(C_{1}\left(V_{w}(0)+K Q(0)\right)\right) \leq\left|\sigma_{\gamma}(0)\right| \exp \left(2 C_{1}\left(V_{w}(0)+K Q(0)\right)\right),
$$

where it is to be understood that when a rarefaction front meets a strong shock, it is "continued" as a shock of the opposite family.

\subsection{Interactions inside a family}

1. No shock/rarefaction interaction inside a family. We only consider the case of the first family, since the case of the second family is treated similarly. Let us suppose that such a meeting happens, and consider the first (in decreasing time) of these meetings: consider, say, the case of a 1-rarefaction front separating $u_{l}$ and $u_{m}$, on the left of a 1shock separating $u_{m}$ and $u_{r}$, the pattern with the shock on the left of the rarefaction front being again treated similarly. Hence $u_{r} \in S_{1}\left(u_{m}\right)$ and $u_{l} \in R_{1}^{l}\left(u_{m}\right)$.

The respective speeds of these fronts are by construction $s\left(u_{l}, u_{m}\right)$ and $s\left(u_{m}, u_{r}\right)$. The fact that these two fronts meet implies that

$$
s\left(u_{m}, u_{r}\right)>s\left(u_{l}, u_{m}\right) .
$$

Clearly, $s$ is a symmetric function, and hence

$$
s\left(u_{m}, u_{r}\right)>s\left(u_{m}, u_{l}\right) .
$$

We remark that in the $(\rho, m)$ plane, the left rarefaction curve $R_{1}^{l}\left(u_{m}\right)$ is above the shock curve $S_{1}\left(u_{m}\right)$ : this follows from the fact that $R_{1}^{l}$ has the same expression as $R_{1}\left(u_{m}\right)$ with $\rho>\rho_{m}$ and from the Cauchy-Schwarz inequality.

Now we introduce the state $\tilde{u}_{r}$ as the intersection in the $(\rho, m)$ plane of the curve $S_{1}\left(u_{m}\right)$ with the interval $\left[u_{m}, u_{l}\right]$. Note that this intersection exists because $R_{1}^{l}\left(u_{m}\right)$ is (strictly) concave, hence $\left[u_{m}, u_{l}\right]$ is strictly below the tangent to $R_{1}^{l}\left(u_{m}\right)$ at $u_{m}$. Consequently, $\left[u_{m}, u_{l}\right]$ is locally below $S_{1}\left(u_{m}\right)$ (which has the same tangent) as well. Hence together with the preceding remark, this proves the existence of $\tilde{u}_{r}$.

Now by construction

$$
s\left(u_{m}, u_{l}\right)=s\left(u_{m}, \tilde{u}_{r}\right)
$$

and hence by $(130)$ we get

$$
s\left(u_{m}, u_{r}\right)>s\left(u_{m}, \tilde{u}_{r}\right) .
$$

Now one sees that $s\left(u_{m}, \cdot\right)$ decreases along the Rankine-Hugoniot curve. Indeed, on the 1-Rankine-Hugoniot curve, one has

$$
s=\frac{m-m_{m}}{\rho-\rho_{m}}=\frac{m_{m}}{\rho_{m}}-\sqrt{\kappa \frac{\rho}{\rho_{m}} \frac{\rho^{\gamma}-\rho_{m}^{\gamma}}{\rho-\rho_{m}}} \text { for } \rho>\rho^{m} .
$$


We deduce that on the $S_{1}\left(u_{m}\right)$ curve, $u_{r}$ is between $\tilde{u}_{r}$ and $u_{m}$. Hence, in the usual Euclidean norm,

$$
\left|u_{m}-u_{r}\right|<\left|u_{m}-\tilde{u}_{r}\right|<\left|u_{m}-u_{l}\right|,
$$

which contradicts our previous estimates on the strengths of the shocks and the rarefaction fronts, at least for large $n$.

2. Noncrossing of rarefaction fronts inside a family. This follows from the GlimmLax theory on the spreading of the rarefaction waves (see [22]). We consider the case of 1 -waves, since the 2-waves can be treated similarly. We consider two consecutive rarefaction fronts, that is, a pair of rarefaction fronts which at the beginning $t=0$ do not enclose any other 1 -front. Note that we do not consider the case when they are separated by a 1-shock since by the previous point and (128), such rarefaction fronts do not collide.

Denote by $\mathcal{C}_{1}$ and $\mathcal{C}_{2}$ the two rarefaction fronts as in Figure 5 , with $X=\mathcal{C}_{1}(0)$ and $Y=\mathcal{C}_{2}(0)$. These curves are contained in $\Sigma^{i}(t)$ when there are two strong shocks, or in a fixed $\Sigma^{+}$or $\Sigma^{-}$when there is only one strong shock, since they do not meet the strong shock of their family, and the meeting with the strong shock of the opposite family destroys them. We suppose that no meeting of fronts of the same family has occurred yet.

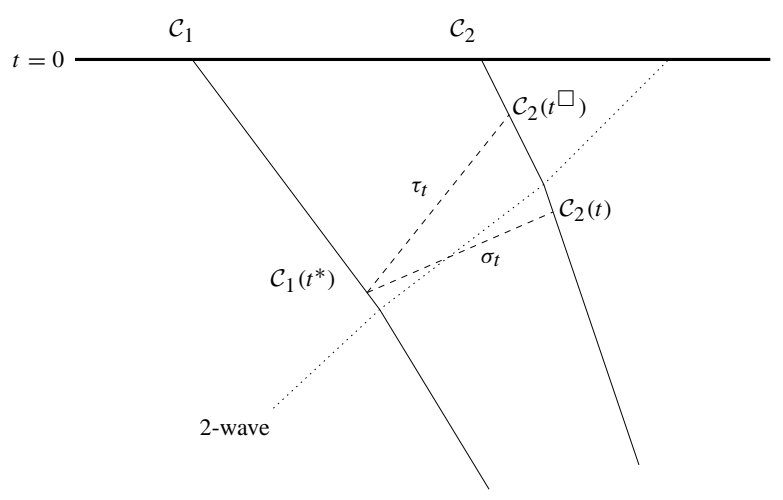

Fig. 5. Focusing of rarefaction fronts.

In what follows, the radius $r$ which measures the oscillation of the solution between the two strong shocks (or on the side of the strong shock which contains the "initial domain" $\{0\} \times[0,1]$ when there is only one strong shock) is taken small enough. Let us underline that $r$ is to be chosen small enough, independently of $n$. As we saw earlier, considering a final state of sufficiently small total variation yields an approximation supported in $\mathfrak{D}$ with $r$ arbitrarily small. All the states considered in this subsection lie in $B\left(\bar{u}_{1} ; r\right)$.

Given $t<0$, we construct two straight lines $\sigma_{t}$ and $\tau_{t}$ as follows. We fix

$$
\check{\lambda}_{i}:=\inf _{u \in B\left(\bar{u}_{1} ; r\right)} \lambda_{i}(u) \quad \text { and } \quad \hat{\lambda}_{i}:=\sup _{u \in B\left(\bar{u}_{1} ; r\right)} \lambda_{i}(u),
$$


and then set

$$
\lambda_{*}=\check{\lambda}_{2}-r \quad \text { and } \quad \lambda^{*}=\hat{\lambda}_{2}+r .
$$

We define $\sigma_{t}$ to pass through $\mathcal{C}_{2}(t)$ with speed $\lambda^{*}$; this curve intersects $\mathcal{C}_{1}$ at the point $\mathcal{C}_{1}\left(t^{*}\right)$. Then we define $\tau_{t}$ to pass through $\mathcal{C}_{1}\left(t^{*}\right)$ with speed $\lambda_{*}$; let $t^{\square}$ be the time at which this curve crosses $\mathcal{C}$ (see Figure 5).

From now on, $\mathcal{C}_{1}$ are $\mathcal{C}_{2}$ are considered as maps $\mathbb{R}^{+} \rightarrow \mathbb{R}$ (mapping $t$ to the position of $\mathcal{C}_{i}(t)$ on the $x$-line). Define the "horizontal" distance between $\mathcal{C}_{2}(t)$ and $\mathcal{C}_{1}\left(t^{*}\right)$ :

$$
D^{*}(t):=\mathcal{C}_{2}(t)-\mathcal{C}_{1}\left(t^{*}\right) .
$$

One has

$$
\frac{d}{d t} D^{*}(t)=\dot{\mathcal{C}}_{2}(t)-\dot{\mathcal{C}}_{1}\left(t^{*}\right) \frac{d t^{*}}{d t} .
$$

Also, $\mathcal{C}_{2}(t)-\mathcal{C}_{1}\left(t^{*}\right)=\lambda_{*}\left(t-t^{*}\right)$ yields

$$
\frac{d t^{*}}{d t}=1+\frac{\dot{\mathcal{C}}_{2}(t)-\dot{\mathcal{C}}_{1}\left(t^{*}\right)}{\dot{\mathcal{C}}_{1}(t)-\lambda_{*}}
$$

In turn, this leads to

$$
\frac{d D^{*}}{d t}=\left(\dot{\mathcal{C}}_{2}(t)-\dot{\mathcal{C}}_{1}\left(t^{*}\right)\right) \frac{\lambda_{*}}{\lambda_{*}-\dot{\mathcal{C}}_{1}(t)}=\left(\dot{\mathcal{C}}_{2}(t)-\dot{\mathcal{C}}_{1}\left(t^{*}\right)\right) \frac{\lambda_{*}}{\lambda_{*}-\underline{\lambda}_{1}}(1+O(r)) .
$$

But $\dot{\mathcal{C}}_{2}(t)-\dot{\mathcal{C}}_{1}\left(t^{*}\right)$ can be estimated by

$$
\dot{\mathcal{C}}_{2}(t)-\dot{\mathcal{C}}_{1}\left(t^{*}\right)=A+B,
$$

where

$$
\begin{aligned}
& A(t):=\left[\dot{\mathcal{C}_{2}}(t)-\lambda_{1}\left(\mathcal{C}_{2}(t)^{-}\right)\right]-\left[\dot{\mathcal{C}_{1}}\left(t^{*}\right)-\lambda_{1}\left(\mathcal{C}_{1}\left(t^{*}\right)^{-}\right)\right], \\
& B(t):=\lambda_{1}\left(\mathcal{C}_{2}(t)^{-}\right)-\lambda_{1}\left(\mathcal{C}_{1}\left(t^{*}\right)^{-}\right) .
\end{aligned}
$$

We have the following estimate on $A$, using (33):

$$
\begin{aligned}
A & =\frac{1}{2}\left[\lambda_{1}\left(\mathcal{C}_{1}\left(t^{*}\right)^{+}\right)-\lambda_{1}\left(\mathcal{C}_{1}\left(t^{*}\right)^{-}\right)\right]+\frac{1}{2}\left[\lambda_{1}\left(\mathcal{C}_{2}(t)^{+}\right)-\lambda_{1}\left(\mathcal{C}_{2}(t)^{-}\right)\right]+o\left(\frac{1}{n^{2}}\right) \\
& =\frac{1}{2} \frac{\partial \lambda_{1}}{\partial w^{2}}\left(\bar{u}_{1}\right)\left(\sigma_{1}\left(t^{*}\right)+\sigma_{2}(t)\right)+o\left(\frac{1}{n^{2}}\right)+O\left(r\left[\sigma_{1}\left(t^{*}\right)+\sigma_{2}(t)\right]\right) .
\end{aligned}
$$

For $B$, we have

$$
\begin{aligned}
B(t) & =\lambda_{1}\left(\mathcal{C}_{2}(t)^{-}\right)-\lambda_{1}\left(\mathcal{C}_{1}\left(t^{*}\right)^{-}\right) \\
& =\sum_{\substack{2 \text {-waves } \\
\text { crossing } \sigma_{t}}} \frac{\partial \lambda_{1}}{\partial w^{1}}\left(\bar{u}_{1}\right)\left[w^{1}\right]+\frac{\partial \lambda_{1}}{\partial w^{2}}\left(\bar{u}_{1}\right)\left[w^{2}\right]+O(r) \sum_{\substack{2 \text {-waves } \\
\text { crossing } \sigma_{t}}}\left|\left[w^{1}\right]\right|+\left|\left[w^{2}\right]\right| .
\end{aligned}
$$

Hence

$$
B(t) \leq C \sum_{\substack{\sigma \text { a } 2 \text {-wave } \\ \operatorname{crossing}\left[\mathcal{C}_{2}\left(t^{\square}\right), \mathcal{C}_{2}(t)\right]}}|\sigma|
$$


We write $\sigma_{1}$ for $\sigma_{1}\left(t^{*}\right)$ and $\sigma_{2}$ for $\sigma_{2}(t)$. We have

$$
\begin{aligned}
D^{*}(t)= & D^{*}(0)+(1+O(r)) \frac{\lambda_{*}}{\lambda_{*}-\underline{\lambda}_{1}} \int_{0}^{t}\left(\frac{1}{2}\left(\sigma_{1}+\sigma_{2}\right) \bar{\xi}_{1}+o\left(\frac{1}{n^{2}}\right)\right) \\
& +O(1) \int_{0}^{t^{*}}\left(\sum_{\substack{\sigma \text { a } 2 \text {-wave } \\
\text { crossing }\left[\mathcal{C}_{2}\left(s^{\square}\right), \mathcal{C}_{2}(s)\right]}}|\sigma|\right) d s .
\end{aligned}
$$

Now in the last term, each wave crossing $\mathcal{C}_{2}$ (at a point $\mathcal{C}(\theta)$ ) is counted when $\mathcal{C}(\theta) \in$ $\left[\mathcal{C}_{2}(s \square), \mathcal{C}_{2}(s)\right]$. Clearly, the time interval for which this happens is of length $O\left(r D^{*}(\theta)\right)$. Hence one gets

$D^{*}(t)=D^{*}(0)+(1+O(r)) \frac{\lambda_{*}}{\lambda_{*}-\underline{\lambda}_{1}} \int_{0}^{t}\left(\frac{1}{2}\left(\sigma_{1}+\sigma_{2}\right) \bar{\xi}_{1}+o\left(\frac{1}{n^{2}}\right)\right)+O(r) \int_{0}^{t} D^{*}(s) d s$.

A simple Gronwall argument yields

$$
\begin{aligned}
D^{*}(t) \geq & \exp (-O(r) t) D^{*}(0) \\
& +(1+O(r)) \int_{0}^{t}\left[\frac{1}{2}\left(\sigma_{1}+\sigma_{2}\right) \frac{\lambda_{*}}{\lambda_{*}-\underline{\lambda}_{1}} \bar{\xi}_{1}+o\left(\frac{1}{n^{2}}\right)\right] \exp (-O(r) \tau) d \tau .
\end{aligned}
$$

Now (102) yields

$$
\begin{aligned}
D^{*}(0) & \geq \lambda^{*} \frac{X-Y}{\lambda^{*}-\lambda_{1}} \\
& \geq \frac{1}{1-\alpha} \frac{\lambda^{*}}{\lambda^{*}-\check{\lambda}_{1}} \frac{\bar{\xi}_{1}}{n^{2}} \max \left(\frac{\bar{\lambda}_{2}-\bar{\lambda}_{1}}{1-Y}, \frac{\bar{\lambda}_{1}}{X}, \frac{-\bar{\lambda}_{1}}{1-Y}\right)^{-1} .
\end{aligned}
$$

Note that using (127), we have

$$
\frac{1}{2}\left(\sigma_{1}+\sigma_{2}\right)=\frac{1+O\left(T V\left(u_{1}\right)\right)}{n^{2}} .
$$

Hence we see that, provided $T V\left(u_{1}\right)$ (and hence also $r$ ) is small enough independently of $n$, and for $n$ large enough,

$$
D^{*}(t)>0 \quad \text { for } t \text { such that }-t<\frac{1}{1-\alpha / 2} \max \left(\frac{\bar{\lambda}_{2}-\bar{\lambda}_{1}}{1-Y}, \frac{\bar{\lambda}_{1}}{X}, \frac{-\bar{\lambda}_{1}}{1-Y}\right)^{-1} .
$$

We consider three cases according to the value of the maximum:

- If the maximum is the first term, then one sees that for some $t$ satisfying (138), the curve $\mathcal{C}_{2}$ has met the strong 2-shock, and hence has ceased to exist. Indeed, from the fact that on both sides of the strong 2 -shock the states are in the domain described in Subsection 5.4 one sees that its speed satisfies

$$
\operatorname{Speed}\left(S_{2}(t)\right) \geq \bar{\lambda}_{2}-\beta,
$$


at least if $r$ is chosen small enough. The curve $\mathcal{C}_{2}$ also has states on both sides in the domain described in Subsection 5.4 hence its speed satisfies

$$
\dot{\mathcal{C}}_{2}(t) \leq \bar{\lambda}_{1}+\beta
$$

Now choosing $\beta$ such that

$$
\frac{1}{\bar{\lambda}_{2}-\bar{\lambda}_{1}-2 \beta}<\frac{1}{1-\alpha / 2} \frac{1}{\bar{\lambda}_{2}-\bar{\lambda}_{1}}
$$

yields the conclusion.

- If the maximum is the second term, then one sees that for some $t$ satisfying (138), the curve $\mathcal{C}_{2}$ has left the domain $[0,1]$ (through 0 ). (Note that this term is useful only when $\bar{\lambda}_{1}>0$.)

- If the maximum is the third term, then one sees that for some $t$ satisfying [138), the curve $\mathcal{C}_{2}$ has left the domain $[0,1]$ (through 1).

This ends the proof that rarefaction fronts do not merge (inside the domain).

\subsection{End of the proof of Proposition 4}

Step 1. Convergence. This is the same argument as in Section 3 The approximations constructed above have a uniform total variation according to the variable $x$, and thanks to 68, a uniform $\operatorname{Lip}_{t}\left(L_{\text {loc }}^{1}\right)$ bound as well. It follows again by Helly's theorem that, up to a subsequence that we do not relabel,

$$
U^{n} \rightarrow \bar{U} \quad \text { in } L_{\mathrm{loc}}^{1}\left((-\infty, 0] \times[0,1] ; \mathbb{R}^{+*} \times \mathbb{R}\right) .
$$

Step 2. Proof that $(\mathrm{EI})$ and the entropy condition are satisfied. Here, we prove that the limit $\bar{U}$ that we obtained satisfies $\mathrm{EI}$ in weak form, and the entropy inequalities. This is not very different from the case of the direct problem with small total variation (see for instance [8, Section 7.4]), but we give the proof for completeness. Moreover, although in this section all shocks travel with exact shock speed, we write the proof in the context where weak fronts can be traveling with a speed which differs from the shock speed by at most $1 / 2^{n}$. Hence, the proof here applies in the context of Section 3 .

We first prove that $\bar{U}$ is a weak solution of $(\mathrm{EI})$. Let $\varphi \in C_{0}^{\infty}((-\infty, 0) \times(0,1) ; \mathbb{R})$ be given. From (140) and the uniform bound on $U^{n}$, we see that it suffices to prove that

$$
I_{n}:=\int_{(-\infty, 0] \times[0,1]}\left(\varphi_{t} U^{n}+\varphi_{x} f\left(U^{n}\right)\right) \rightarrow 0 \quad \text { as } n \rightarrow \infty .
$$

Assuming that $\varphi=0$ for $t \leq-\bar{T}$, and denoting $x_{\alpha}$ the curve corresponding to the front $\alpha$, we have, by Stokes' formula,

$I_{n}=\int_{-\bar{T}}^{0} \sum_{\alpha} \varphi\left(t, x_{\alpha}\right)\left\{\dot{x}_{\alpha}(t)\left(U^{n}\left(t, x_{\alpha}^{+}\right)-U^{n}\left(t, x_{\alpha}^{-}\right)\right)-\left(f\left(U^{n}\left(t, x_{\alpha}^{+}\right)\right)-f\left(U^{n}\left(t, x_{\alpha}^{-}\right)\right)\right)\right\} d t$. 
Denote by $\mathcal{I}_{\alpha}$ the term of the sum corresponding to the wave $\alpha$. We write $[\phi]\left(x_{\alpha}\right)$ for $\phi\left(x_{\alpha}^{+}\right)-\phi\left(x_{\alpha}^{-}\right)$. The term $\mathcal{I}_{\alpha}$ depends on the type of the wave $\alpha$ :

- If $\alpha$ is a strong shock, it moves at the exact Rankine-Hugoniot speed, hence $\mathcal{I}_{\alpha}=0$.

- If $\alpha$ is a weak shock, it moves at the exact speed, up to $2^{-n}$, hence $\mathcal{I}_{\alpha}=O(1) 2^{-n}\left|\sigma_{\alpha}\right|$.

- If $\alpha$ is a rarefaction front (of the family $i$ ), it is easy to see that

$$
[f(u)]\left(x_{\alpha}(t)\right)-s\left(u\left(x_{\alpha}(t)^{-}\right), u\left(x_{\alpha}(t)^{+}\right)\right)[u]\left(x_{\alpha}(t)\right)=O\left(\left|\sigma_{\alpha}\right|^{2}\right),
$$

which yields

$$
\mathcal{I}_{\alpha}=O(1)\left(\frac{1}{n^{2}}+\frac{1}{2^{n}}\right)\left|\sigma_{\alpha}\right| .
$$

If we use the uniform bound on the total strength of the fronts, this leads to the fact that $I_{n} \rightarrow 0$, which was to be proved.

We now turn to the entropy inequality. We consider an entropy/entropy flux pair $(\eta, q)$, with $\eta$ convex. In order to prove (5), it suffices to prove that

$$
\liminf J_{n} \geq 0, \quad \text { where } \quad J_{n}:=\int_{(-\infty, 0] \times \mathbb{R}}\left(\varphi_{t} \eta\left(U^{n}\right)+\varphi_{x} q\left(U^{n}\right)\right) .
$$

As previously, we have

$$
\begin{aligned}
J_{n} & =\int_{-\bar{T}}^{0} \sum_{\alpha} \varphi\left(t, x_{\alpha}\right)\left\{\dot{x}_{\alpha}(t)\left[\eta\left(U^{n}\right)\right]\left(x_{\alpha}(t)\right)-\left[q\left(U^{n}\right)\right]\left(x_{\alpha}(t)\right)\right\} d t \\
& \geq \int_{-\bar{T}}^{0} \sum_{\substack{\alpha \\
\text { weak wave }}} \varphi\left(t, x_{\alpha}\right)\left\{\dot{x}_{\alpha}(t)\left[\eta\left(U^{n}\right)\right]\left(x_{\alpha}(t)\right)-\left[q\left(U^{n}\right)\right]\left(x_{\alpha}(t)\right)\right\} d t .
\end{aligned}
$$

Indeed, the strong shock waves (which travel at exact speed) satisfy the entropy condition (see for instance [1, Lemma 4.1])

$$
s\left[\eta\left(U^{n}\right)\right]\left(x_{\alpha}^{+}(t)\right)-\left[q\left(U^{n}\right)\right]\left(x_{\alpha}(t)\right) \geq 0 .
$$

This is seen by differentiating the above left hand side along the shock curve. Set $u^{+}:=$ $U^{n}\left(t, x_{\alpha}^{+}\right)$and $u^{-}:=U^{n}\left(t, x_{\alpha}^{-}\right), u^{+}=\Phi_{i}\left(\eta, u^{-}\right)$. We have

$$
\begin{aligned}
\frac{d}{d \eta}(s[\eta]-[q]) & =\frac{d s}{d \eta}\left(\eta\left(u^{+}\right)-\eta\left(u^{-}\right)\right)+s D\left(\eta\left(u^{+}\right)\right) \frac{d u^{+}}{d \eta}-D q\left(u^{+}\right) \frac{d u^{+}}{d \eta} \\
& =\frac{d s}{d \eta}\left(\eta\left(u^{+}\right)-\eta\left(u^{-}\right)\right)+D\left(\eta\left(u^{+}\right)\right)\left[s \frac{d u^{+}}{d \eta}-D f\left(u^{+}\right) \frac{d u^{+}}{d \eta}\right] \\
& =\frac{d s}{d \eta}\left(\eta\left(u^{+}\right)-\eta\left(u^{-}\right)\right)-D\left(\eta\left(u^{+}\right)\right)\left[\frac{d s}{d \eta}\left(u^{+}-u^{-}\right)\right],
\end{aligned}
$$

where we differentiated the Rankine-Hugoniot relation in the last step. Now we use the fact that $d s / d \eta<0$ globally along the shock curve (which is easily checked using (30)(31) and the convexity of $\eta$; this yields (142).

Again, denote by $\mathcal{J}_{\alpha}$ the general term in 141 , which depends on the type of the wave $\alpha$. 
- If $\alpha$ is a weak shock, it moves at the exact speed, up to $2^{-n}$, hence $\mathcal{J}_{\alpha} \geq-O(1) 2^{-n}\left|\sigma_{\alpha}\right|$.

- If $\alpha$ is a rarefaction front (of the family $i$ ), it follows all the same from 26)-27) that

$$
\left[q\left(U^{n}\right)\right]\left(x_{\alpha}(t)\right)-s\left(U^{n}\left(x_{\alpha}(t)^{-}\right), U^{n}\left(x_{\alpha}(t)^{+}\right)\right)[\eta(u)]\left(x_{\alpha}(t)\right)=O\left(\left|\sigma_{\alpha}\right|^{2}\right),
$$

which yields

$$
\mathcal{J}_{\alpha} \geq-O(1)\left(\frac{1}{n^{2}}+\frac{1}{2^{n}}\right)\left|\sigma_{\alpha}\right| .
$$

Using the uniform bound on the total strength and the estimate on the size of the rarefaction front, this yields $\lim \inf J_{n} \geq 0$, which was to be proved.

Step 3. Proof that $u_{\mid t=-T_{3}}$ is constant. Let us prove that for $t$ sufficiently negative, there are no fronts inside the domain $[0,1]$, for all the approximations $U^{n}$. Then the function $u$ obtained as a limit is constant for $t$ sufficiently negative.

It follows from the fact that the states considered in the approximations are in the domain $\mathfrak{D}$ defined in Subsection 5.4 that the strong 1-shock leaves it through $x=1$ and that the strong 2-shock leaves the domain through $x=0$, before a time $-T_{3}^{a}$ easily computable. As a consequence, if there is a front inside the domain for times $t \leq-T_{3}^{a}$, it lies in $\Sigma^{i}(t)$.

We note that a front under the two strong shocks is necessarily a shock: a rarefaction front cannot meet the strong shock of its own family, and the meeting with the front of the other family destroys it. Then, any shock-shock interaction is solved in terms of two shocks (as seen in Subsection 5.3). Recall that there are no backward interactions of fronts of the same family. Consequently, one can follow each front under the strong shocks, as a front of a fixed family, and no new fronts appear. Using the definition of the domain in Subsection 5.4 (the states close to the critical curves are avoided), one sees that these fronts must leave the domain.

\section{The Lagrangian case}

\subsection{Introduction}

In this section we prove Theorem 2 The structure of the proof is the same as the one of Theorem 1 in a first step, one shows that one can drive the system from $u_{0}$ to a constant state, then in a second step, one proves that it is possible to travel between arbitrary constant states, and finally in the last step, it is proved that there exists a solution starting from a constant state and reaching $u_{1}$. Only the first step is really different from the one in the proof of Theorem 1 Hence the goal of this section is to prove:

Proposition 5. Let $u_{0}$ be as in Theorem 2 Then there exist $\omega \in \mathbb{R}^{+*} \times \mathbb{R}$ and an entropy solution $u$ of $(\mathrm{P})$ in $[0,1] \times[0,1]$ such that

$$
\begin{aligned}
& u_{\mid t=0}=u_{0}, \\
& u_{\mid t=1}=\omega .
\end{aligned}
$$


Before proving Proposition 5, let us recall that if we write the state $u=(\tau, v)$, the system (P) has eigenvalues $\lambda_{1}=-\sqrt{\kappa \gamma \tau^{-\gamma-1}}$ and $\lambda_{2}=\sqrt{\kappa \gamma \tau^{-\gamma-1}}$ with respective eigenvectors

$$
\begin{aligned}
& r_{1}(u)=\frac{2}{\sqrt{\kappa \gamma}(\gamma+1)} \tau^{(\gamma+3) / 2}\left(\begin{array}{c}
1 \\
-\lambda_{1}(u)
\end{array}\right), \\
& r_{2}(u)=-\frac{2}{\sqrt{\kappa \gamma}(\gamma+1)} \tau^{(\gamma+3) / 2}\left(\begin{array}{c}
1 \\
-\lambda_{2}(u)
\end{array}\right),
\end{aligned}
$$

normalized in order that $\nabla \lambda_{i} \cdot r_{i}=1$. The rarefaction curves are

$$
R_{i}: v-v_{-}=(-1)^{i} \frac{2 \sqrt{\kappa \gamma}}{\gamma-1}\left[\tau^{-(\gamma-1) / 2}-\tau_{-}^{-(\gamma-1) / 2}\right] \quad \text { with } \quad(-1)^{i}\left(\tau-\tau_{-}\right)<0
$$

and the shock curves are

$$
S_{i}: v-v_{-}=\sqrt{-\kappa\left(\tau^{-\gamma}-\tau_{-}^{-\gamma}\right)\left(\tau-\tau_{-}\right)} \quad \text { with } \quad(-1)^{i}\left(\tau-\tau^{-}\right)>0
$$

We parameterize Lax's wave curves (that we still denote $\left.\Phi_{i}\right)$ by $\lambda\left(u_{r}\right)$ for the rarefactions and by $2\left(\lambda\left(u_{r}\right)-s\right)$ for the shocks, which makes them $C^{2}$-regular (see [26]). Again we put an " $l$ " exponent for left curves. When $\left(u_{1}, u_{2}\right)$ determines an $i$-wave, $u_{2}=\Phi_{i}\left(\sigma\left(u_{1}, u_{2}\right), u_{2}\right)$, we take $\left|\sigma\left(u_{1}, u_{2}\right)\right|$ as a measure of the strength of this wave.

As previously, when $\tau_{1} \neq \tau_{2}$, we write

$$
s\left(u_{1}, u_{2}\right)=-\frac{v_{1}-v_{2}}{\tau_{1}-\tau_{2}},
$$

whether $\left(u_{1}, u_{2}\right)$ determines a shock or not. It is again elementary to establish that on a rarefaction curve $u^{+}=R_{i}\left(s, u^{-}\right), s>0$, we have

$$
s\left(u^{-}, u^{+}\right)=\frac{\lambda_{i}\left(u^{-}\right)+\lambda_{i}\left(u^{+}\right)}{2}+O\left(\left|u^{-}-u^{+}\right|^{2}\right) \quad \text { and } \quad \lambda\left(u^{-}\right)<s<\lambda\left(u^{+}\right) .
$$

Finally, recall that the Riemann invariants here are given by (7).

We begin the proof of Proposition 5 by introducing a domain for the solution that we are going to construct.

\subsection{Domain for the solution}

As for Theorem 1 , one of the ideas is to let a strong 2-shock enter the domain. We begin by determining the shock. We consider $U_{0}^{-}$such that $\left(U_{0}^{-}, \bar{u}_{0}\right)$ is a 2-shock of speed greater than 3 and such that $\lambda_{1}\left(U_{0}^{-}\right) \leq-3$. This is possible since by 147 the shock speed is given by

$$
s=\sqrt{-\kappa \frac{\left(\bar{\tau}_{0}\right)^{-\gamma}-\left(\tau_{0}^{-}\right)^{-\gamma}}{\bar{\tau}_{0}-\tau_{0}^{-}}} \rightarrow+\infty \quad \text { as } \tau_{0}^{-} \rightarrow 0^{+},
$$


where $U_{0}^{-}=\left(\tau_{0}^{-}, v_{0}^{-}\right)$and $\bar{u}_{0}=\left(\bar{\tau}_{0}, \bar{v}_{0}\right)$, and since

$$
\lambda_{1}\left(U_{0}^{-}\right)=-\sqrt{\kappa \gamma}\left(\tau_{0}^{-}\right)^{(-\gamma-1) / 2} \rightarrow-\infty \quad \text { as } \tau_{0}^{-} \rightarrow 0^{+} .
$$

To fix notations, we write $\bar{u}_{0}=\Phi_{2}\left(\bar{p}, U_{0}^{-}\right)$.

Now the domain considered in what follows has the form

$$
\mathfrak{D}=B\left(U_{0}^{-} ; r\right) \cup B\left(\bar{u}_{0} ; r\right),
$$

where $r$ is small enough that: the vacuum is avoided, Glimm's estimates are satisfied in each component of $\mathfrak{D}$, the speeds are strictly separated in each component of $\mathfrak{D}$, any simple wave leading from a state of $B\left(U_{0}^{-} ; r\right)$ to a state of $B\left(\bar{u}_{0} ; r\right)$ is a 2-shock with speed greater than 2 , and a state $\omega \in B\left(U_{0}^{-} ; r\right)$ satisfies $\lambda_{1}(\omega) \leq-2$. Other conditions on $r$ are found in the next lemmas.

Remark 8. One could have used an arbitrarily small shock here, but we underline that the constant $r$ described above depends on the reference shock and tends rapidly to 0 as the shock shrinks. Also, we would not be able to require $\lambda_{1}(\omega) \leq-2$ and the speed of the strong shock to be greater than 2 , but rather $\lambda_{1}(\omega) \leq-c<0$ and the speed of the strong shock to be greater than $c>0$ (and the time of controllability $T$ is of course affected). Note also that one can make the same type of construction for (EI) provided the base point $\bar{u}_{0}$ satisfies $\lambda_{1}\left(\bar{u}_{0}\right)<0<\lambda_{2}\left(\bar{u}_{0}\right)$.

\subsection{Two lemmas}

Before constructing the front-tracking approximations for a solution to Proposition 5, we establish two preliminary lemmas. The general idea of these lemmas is to prove that, if we are able to send supplementary 2-shocks towards the strong shock at the right time and with the right strength, one can get rid of the 1-shocks that would naturally emerge from the strong shock. These shocks are the principal obstruction to reaching a constant state.

Lemma 11. If $r$ is small enough then the following holds. Let $u_{l} \in B\left(U_{0}^{-} ; r\right)$ and $u_{m}, u_{r} \in B\left(\bar{u}_{0} ; r\right)$ be such that

$$
\left(u_{l}, u_{m}\right) \text { is a 2-shock and }\left(u_{m}, u_{r}\right) \text { is a 1-shock. }
$$

Then there exists $\tilde{u}_{l}$ such that

$$
\left(\tilde{u}_{l}, u_{l}\right) \text { is a 2-shock and }\left(\tilde{u_{l}}, u_{r}\right) \text { is a 2-shock, }
$$

and moreover

$$
\begin{aligned}
& \sigma\left(\tilde{u}_{l}, u_{l}\right)=O\left(\sigma\left(u_{m}, u_{r}\right)\right), \\
& \sigma\left(\tilde{u}_{l}, u_{r}\right)=\sigma\left(\tilde{u}_{l}, u_{m}\right)+O\left(\sigma\left(u_{m}, u_{r}\right)\right) .
\end{aligned}
$$


Lemma 12. If $r$ is small enough then the following holds. Consider $u_{l} \in B\left(U_{0}^{-} ; r\right)$ and $u_{m}, u_{r} \in B\left(\bar{u}_{0} ; r\right)$ such that

$$
\left(u_{l}, u_{m}\right) \text { is a 2-shock and }\left(u_{m}, u_{r}\right) \text { is a 2-rarefaction front. }
$$

Then there exists $\tilde{u}_{l}$ such that

$$
\left(\tilde{u}_{l}, u_{l}\right) \text { is a 2-shock and }\left(\tilde{u_{l}}, u_{r}\right) \text { is a 2-shock, }
$$

and moreover

$$
\begin{aligned}
& \sigma\left(\tilde{u}_{l}, u_{l}\right)=O\left(\sigma\left(u_{m}, u_{r}\right)\right) \\
& \sigma\left(\tilde{u}_{l}, u_{r}\right)=\sigma\left(u_{l}, u_{m}\right)+O\left(\sigma\left(u_{m}, u_{r}\right)\right)
\end{aligned}
$$
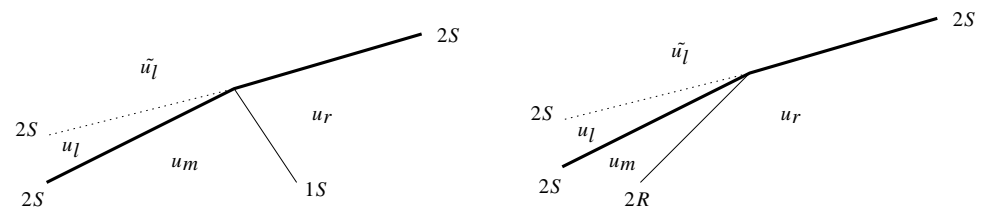

Fig. 6. Lemmas 11 and 12

Proof of Lemmas 11 and 12 . We begin with Lemma 11 To fix the notations, we write $u_{m}=\Phi_{2}\left(p, u_{l}\right), u_{r}=\Phi_{1}\left(\sigma_{1}, u_{m}\right), u_{l}=\Phi_{2}\left(\sigma_{2}, \tilde{u_{l}}\right)$, and $s$ the speed of the shock $\left(u_{l}, u_{m}\right)$. By Lemma 1, the Riemann problem $\left(\tilde{u}_{l}, u_{r}\right)$ is solvable, at least if $r$ is small enough. Let $\left(\sigma_{1}^{\prime}, p^{\prime}\right)$ be the strengths of the resulting waves, that is,

$$
u_{r}=\Phi_{2}\left(p^{\prime}, \Phi_{1}\left(\sigma_{1}^{\prime}, \tilde{u}_{l}\right)\right)
$$

We consider the mapping

$$
(F, G):\left(u_{l}, \sigma_{1}, \sigma_{2}, p\right) \mapsto\left(\sigma_{1}^{\prime}, p^{\prime}\right) .
$$

Then $F\left(u_{l}, 0,0, p\right)=0$ for $u_{l} \in B\left(U_{0}^{-} ; r\right)$ and $p$ in a neighborhood of $\bar{p}$. It follows from Lemma1 1 that

$$
\left[\partial_{\sigma_{2}} F\right]\left(u_{l}, 0,0, p\right)=\frac{\lambda_{2}\left(u_{l}\right)-s}{\lambda_{1}\left(u_{l}\right)-s} \cdot \frac{\operatorname{det}\left(r_{2}\left(u_{l}\right), u_{m}-u_{l}\right)}{\operatorname{det}\left(r_{1}\left(u_{l}\right), u_{m}-u_{l}\right)} .
$$

Now we remark that $u_{m}-u_{l}=\left(\tau_{m}-\tau_{l}\right)^{t}(1,-s)$ and

$$
\begin{aligned}
& r_{2}\left(u_{l}\right)=-\frac{2}{\sqrt{\kappa \gamma}(\gamma+1)} \tau_{l}^{(\gamma+3) / 2 t}\left(1,-\lambda_{2}\left(u_{l}\right)\right), \\
& r_{1}\left(u^{l}\right)=\frac{2}{\sqrt{\kappa \gamma}(\gamma+1)} \tau_{l}^{(\gamma+3) / 2 t}\left(1,-\lambda_{1}\left(u_{l}\right)\right) .
\end{aligned}
$$


By Lax's inequalities, one has $\tau_{m}>\tau_{l}, \lambda_{2}\left(u_{m}\right)<s<\lambda_{2}\left(u_{l}\right)$ and $s>\lambda_{1}\left(u_{l}\right)$. We get

$$
\left[\partial_{\sigma_{2}} F\right]\left(u_{l}, 0,0, p\right)=-\frac{\lambda_{2}\left(u_{l}\right)-s}{\lambda_{1}\left(u_{l}\right)-s} \cdot \frac{\lambda_{2}\left(u_{l}\right)-s}{\lambda_{1}\left(u_{l}\right)-s}<0 .
$$

Hence the equation $F\left(u_{l}, \sigma_{1}, \cdot, p\right)=0$ can be solved for $\left(u_{l}, \sigma_{2}, p\right)$ in a neighborhood of $\left(U_{0}^{-}, 0, \bar{p}\right)$. Moreover,

$$
\left[\partial_{\sigma_{1}} F\right]\left(u_{l}, 0,0, p\right)=\frac{\lambda_{1}\left(u_{m}\right)-s}{\lambda_{1}\left(u_{l}\right)-s} \cdot \frac{\operatorname{det}\left(r_{1}\left(u_{m}\right), u_{m}-u_{l}\right)}{\operatorname{det}\left(r_{1}\left(u_{l}\right), u_{m}-u_{l}\right)} .
$$

Hence by Lax's inequalities,

$$
\left[\partial_{\sigma_{1}} F\right]\left(u_{l}, 0,0, p\right)>0 .
$$

If $r$ is chosen small enough so that the above derivatives are bounded and bounded away from 0 , the existence of $\sigma_{2}$ and (153) and 154 follow from the local inversion theorem. Moreover the solution $\sigma_{2}$ is negative when $\sigma_{1}<0$, which implies that the wave $\left(\tilde{u}_{l}, u_{l}\right)$ is indeed a shock. Hence this establishes Lemma 11

The proof of Lemma 12 is entirely similar with $u_{r}=\Phi_{1}\left(\sigma_{1}, u_{m}\right), \sigma_{1}<0$, replaced by $u_{r}=\Phi_{2}\left(\varsigma_{2}, u_{m}\right), \varsigma_{2}>0$. Then defining again

$$
(F, G):\left(u_{l}, \varsigma_{2}, \sigma_{2}, p\right) \mapsto\left(\sigma_{1}^{\prime}, p^{\prime}\right),
$$

one gets

$$
\left[\partial_{\varsigma_{2}} F\right]\left(u_{l}, 0,0, p\right)=\frac{\lambda_{2}\left(u_{m}\right)-s}{\lambda_{1}\left(u_{l}\right)-s} \cdot \frac{\operatorname{det}\left(r_{2}\left(u_{m}\right), u_{m}-u_{l}\right)}{\operatorname{det}\left(r_{1}\left(u_{l}\right), u_{m}-u_{l}\right)}<0,
$$

which yields the conclusion.

Remark 9. Note in passing that one recovers by (156)-157) and (158) the fact that, at least for small waves, the interaction of a 1-shock (resp. 1-rarefaction front, 2-shock, 2-rarefaction front) with the strong 2-shock generates a shock (resp. rarefaction front, rarefaction front, shock) in the first family. This is also true for (EI), and Lemmas 11 and 12 could also be proven in that case.

\subsection{Construction of front-tracking approximations}

In this subsection, we explain the construction of front-tracking approximations of a solution to Proposition 5. This is done in two steps: first we construct an approximate solution "before the interaction with the strong shock", and then we complete the approximation "after the interaction with the strong shock".

We begin by introducing approximations $u_{0}^{n}$ of the initial state $u_{0}$, on $[0,1]$, satisfying:

$$
\begin{aligned}
& T V\left(u_{0}^{n}\right) \leq T V\left(u_{0}\right), \\
& u_{0}^{n} \rightarrow u_{0} \text { in } L^{1}((0,1)), \\
& u_{0}^{n} \text { is piecewise constant. }
\end{aligned}
$$




\section{Step 1.}

a. For fixed $n$, we locally solve approximately the Riemann problem for any discontinuity point in $(0,1)$ (that is, with rarefaction waves replaced by rarefaction fans with accuracy $1 / n$ as described in Section 3 .

b. At point 0 , we solve the Riemann problem $U_{0}^{-}, u_{0}^{n}\left(0^{+}\right)$; we keep only the 2-wave (which is a 2-shock if $n$ is large enough that $u_{0}^{n}\left(0^{+}\right) \in B\left(\bar{u}_{0} ; r\right)$ ). This front is called strong.

c. We extend different fronts to their first interaction point. Here the convention is that an interaction point is either the intersection of two fronts, or the crossing of a front with the boundary $[0,+\infty) \times\{0\} \cup[0,+\infty) \times\{1\}$. We modify if necessary the speeds of the waves by an amount $1 / 2^{n}$ in order that, at a given time, at most one interaction takes place, involving either two fronts, or one front and the boundary. We denote by $\hat{u}^{n}$ the resulting piecewise constant function that we construct progressively.

Now we explain how we extend $\hat{u}^{n}$ past interaction points. This depends on the nature of the interaction. We suppose that the states on the left of the strong shock lie in $B\left(U_{0}^{-} ; r\right)$, and those on the left in $B\left(\bar{u}_{0} ; r\right)$.

1. Weak-weak interaction. If two weak fronts $\left(u_{l}, u_{m}\right)$ and $\left(u_{m}, u_{r}\right)$ interact, then we extend the solution by the approximate Riemann solution of the problem $\left(u_{l}, u_{r}\right)$, with the convention that we do not split rarefaction fronts again.

2. Front/boundary interaction. When a front meets the boundary, we extend the solution by just ending the front (say, for instance, if the front $\left(u_{l}, u_{m}\right)$ meets the boundary $\{0\}$ at time $t$, then $\hat{u}^{n}$ is extended for times larger than $t$ by $\left.u_{m}\right)$.

3. Strong/weak interaction. We suppose that the strong wave $\left(u_{l}, u_{m}\right)$ interacts with the weak wave $\left(u_{m}, u_{r}\right)$ on its right (with $u_{l} \in B\left(U_{0}^{-} ; r\right)$ and $\left.u_{m}, u_{r} \in B\left(\bar{u}_{0} ; r\right)\right)$. Then $\left(u_{l}, u_{m}\right)$ is a strong 2-shock, say $u_{m}=\Phi_{2}\left(p, u_{l}\right)$. We discuss the various extensions of $\hat{u}^{n}$ according to the nature of the weak wave $\left(u_{m}, u_{r}\right)$.

$\alpha$. $\left(u_{m}, u_{r}\right)$ is a 1-rarefaction front. In that case, by Schochet's lemma, there exist $\varepsilon_{1}^{\prime}$ and $\varepsilon_{2}^{\prime}$ such that

$$
u_{r}=\Phi_{2}\left(p+\varepsilon_{2}^{\prime}, \Phi_{1}\left(\varepsilon_{1}^{\prime}, u_{l}\right)\right)
$$

with the estimates

$$
\left|\varepsilon_{2}^{\prime}\right|+\left|\varepsilon_{1}^{\prime}\right|=O\left(\sigma\left(u_{m}, u_{r}\right)\right) .
$$

Moreover, it follows from the expression of Schochet's matrix (see also (157)) that $\varepsilon_{1}^{\prime}>0$. We fix $\tilde{u}_{l}:=\Phi_{1}\left(\varepsilon_{1}^{\prime}, u_{l}\right)$. Then we extend the approximation $\hat{u}^{n}$ past the interaction point by $u_{l}$ and $\tilde{u}_{l}$ separated by a horizontal line on the left, and $\tilde{u}_{l}$ and $u_{r}$ separated by a single jump at speed $s\left(\tilde{u}_{l}, u_{m}\right)$. (See Figure 7 )

Hence in the above construction, there is a horizontal discontinuity between $u_{l}$ and $\tilde{u}_{l}$, which is certainly not intended to satisfy equation $[\mathrm{P}]$ even approximately. This is modified in the second step of the construction.

$\beta$. $\left(u_{m}, u_{r}\right)$ is a 2-shock. Still by Schochet's lemma, there are some $\varepsilon_{1}^{\prime}$ and $\varepsilon_{2}^{\prime}$ such that

$$
u_{r}=\Phi_{2}\left(p+\varepsilon_{2}^{\prime}, \Phi_{1}\left(\varepsilon_{1}^{\prime}, u_{l}\right)\right)
$$


with the estimate $(160)$ still fulfilled. Moreover, it follows from the expression of Schochet's matrix (see also 158 ) that $\varepsilon_{1}^{\prime}>0$. Then we extend $\hat{u}^{n}$ past the time of interaction as in the previous case.

$\gamma$. $\left(u_{m}, u_{r}\right)$ is a 1-shock. Here we use Lemma 11 . Hence there is some $\tilde{u}_{l}$ such that $u_{l}=\Phi_{2}\left(\alpha_{2}, \tilde{u}_{l}\right), \alpha_{2}<0$, and $u_{r}=\Phi_{2}\left(p+\varepsilon_{2}^{\prime}, \tilde{u}_{l}\right)$. Recall that we have estimates (151) and (152). Then we extend $\hat{u}^{n}$ past the time of interaction as previously, with a horizontal discontinuity between $u_{l}$ and $\tilde{u}_{l}$.

$\delta$. $\left(u_{m}, u_{r}\right)$ is a 2-rarefaction. Here we use Lemma 12 Again there is some $\tilde{u}_{l}$ such that $u_{l}=\Phi_{2}\left(\alpha_{2}, \tilde{u}_{l}\right), \alpha_{2}<0$, and $u_{r}=\Phi_{2}\left(p+\varepsilon_{2}^{\prime}, \tilde{u}_{l}\right)$. Then we extend $\hat{u}^{n}$ past the time of interaction as previously, with a horizontal discontinuity between $u_{l}$ and $\tilde{u}_{l}$. Here we have estimates 153 and (154).

In each case, the 2-wave outgoing from the interaction point is called strong. All other waves are called weak.

Let us remark that there is no interaction on the left of the strong shock since there are only horizontal discontinuity lines there. The first step is represented in Figure 7 .

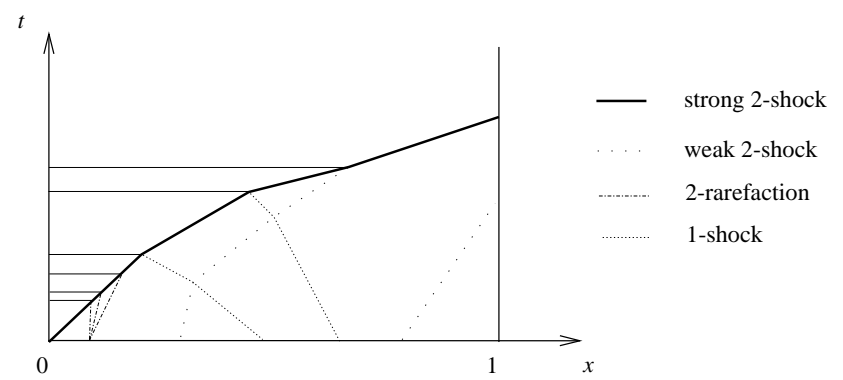

Fig. 7. Step 1.

If we assume for the moment that there are only a finite number of fronts and of interaction points, and that all states considered on the left of the strong wave lie in $B\left(U_{0}^{-} ; r\right)$ and all states on the right of it lie in $B\left(\bar{u}_{0} ; r\right)$, the above algorithm is well-defined. Moreover, the strong wave is a 2 -shock of speed greater than 2 and hence it has left the domain before $t=1 / 2$.

Now we modify the approximation "after the strong shock". The goal is to make it a suitable approximation of an entropy solution, intended to satisfy (144).

Step 2. We denote by $S(t)$ the position of the strong 2-shock at time $t$. We let $\mathfrak{S}(x)$ be the time when the strong 2 -shock reaches $x \in[0,1]$. We define $T_{I}:=\mathfrak{S}(1)$. The goal is to reconstruct properly the above approximation on the domain

$$
\Omega=\bigcup_{x \in[0,1]}[\mathfrak{S}(x),+\infty) \times\{x\} .
$$

The main point is to consider $1-x$ as time, and $[\mathfrak{S}(x),+\infty)$ as the space domain. Hence we get a problem in a varying domain, with boundary conditions on the "moving bound- 


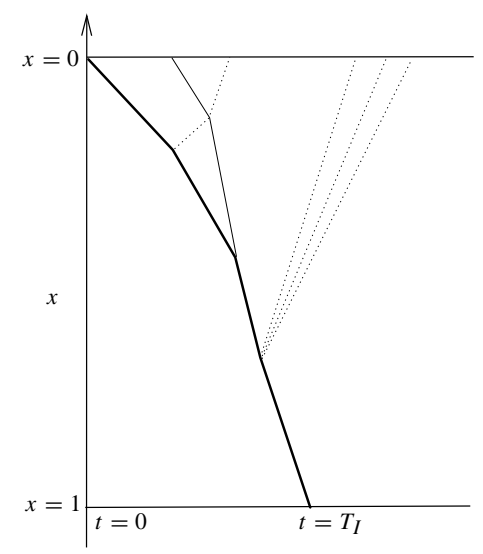

Fig. 8. Second step.

ary" $\mathfrak{S}(x)$, as described in Figure 8 The new piecewise constant function constructed in this section will be denoted $U^{n}$.

We let $t_{1}, \ldots, t_{k}$ be the different times of interaction of a weak shock with the strong shock that occurred in the previous step, in increasing order. We denote by $x_{1}, \ldots, x_{k}$ the corresponding positions in $[0,1]$. We add $t_{k+1}=T_{I}, x_{k+1}=1, t_{0}=0$ and $x_{0}=0$. For each $\left[t_{i}, t_{i+1}\right), i=0, \ldots, k$, there is a state $\tilde{u}_{i+1}$ on the left of the strong shock (in the $(t, x)$ plane, or on its right in the $(1-x, t)$ plane) constructed in the above algorithm.

We start from $U^{n}=\tilde{u}_{k+1}$ on $\left[T_{I},+\infty\right.$ ) at "time $1-x=0$ " (hence there is no front in the domain). We let the fronts evolve in the domain (at the beginning, there are none), until one of the following two situations occurs: either $1-x$ reaches $1-x_{i}$, or two fronts meet in the domain $\Omega$.

First situation. For each $i=k, \ldots, 1$, we have the following alternative, as seen in the construction of Step 1: either

- $\tilde{u}_{i+1}=\Phi_{2}\left(\varepsilon_{2}^{i}, \tilde{u}_{i}\right)$ for some $\varepsilon_{2}^{i}<0$ (this corresponds to cases $\gamma$ and $\delta$ of Step 1), or

- $\tilde{u}_{i+1}=\Phi_{1}\left(\varepsilon_{1}^{i}, \tilde{u}_{i}\right)$ for some $\varepsilon_{1}^{i}>0$ (this corresponds to cases $\alpha$ and $\beta$ of Step 1).

In the first case, we extend the function $U^{n}$ over "time" $1-x=1-x_{i}$ on the right of the strong 2 -shock (in $(t, 1-x)$ plane) by $\tilde{u}_{i}$ and $\tilde{u}_{i+1}$, separated by a backward-in-time 2 -front (which is a shock when seen in the usual direction of time) at shock speed, that is, by the straight line passing through $\left(t_{i}, x_{i}\right)$, with equation

$$
\left(x-x_{i}\right)+\left(t-t_{i}\right) s\left(\tilde{u}_{i}, \tilde{u}_{i+1}\right)=0, \quad t \leq t_{i} .
$$

Note that this front enters the domain (161) by Lax's inequalities.

In the second case, we distinguish two possibilities:

- For $x_{i}$ that corresponds to the action of a 1-rarefaction front in Step 1, that is, in case $\alpha$ above, we extend the function $U^{n}$ past time $x=x_{i}$ by $\tilde{u}_{i}$ and $\tilde{u}_{i+1}$, separated by a 
forward-in-time 1-front (which is a rarefaction front) at shock speed, that is, again by the straight line passing through $\left(t_{i}, x_{i}\right)$, with equation

$$
\left(x-x_{i}\right)+\left(t-t_{i}\right) s\left(\tilde{u}_{i}, \tilde{u}_{i+1}\right)=0, \quad t \geq t_{i} .
$$

- For $x_{i}$ that corresponds to the action of a 2-shock in Step 1, that is, in case $\beta$ above, we extend the function $U^{n}$ beyond time $1-x=1-x_{i}$ by a (forward-in-time) rarefaction fan of accuracy $1 / n$ from $\tilde{u}_{i}$ to $\tilde{u}_{i+1}$, each (rarefaction) front evolving at shock speed.

Note that these fronts lie in $\Omega$, because they evolve forward in time (with negative speed).

Here we need not modify the speeds of the fronts, since, as we will see, there can only be binary interactions. Simultaneous interactions (in different places) can be treated as successive interactions.

Second situation. We extend the different fronts that enter the domain and let them evolve at constant speed in the domain as $1-x$ increases until two of these fronts meet.

Let us remark that in this scheme, in the domain (161), two 1-fronts do not meet because they are 1-rarefaction fronts evolving forward in time (see (148), and two 2-fronts do not meet because they are 2-shock fronts evolving backward in time, as a consequence of Lax's inequalities. (Note that, in particular, 2-shocks do not meet $\mathfrak{S}$ once they have entered the domain.) Consequently, there can only be binary interactions. As a result, one just has to deal with the meeting of a 1-rarefaction front with a 2-shock, as described in Figure 9.

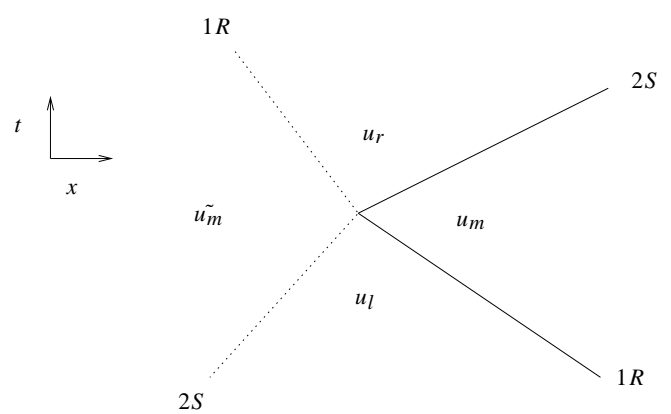

Fig. 9. Interactions in the second step.

We consider a 2-shock $\left(u_{r}, u_{m}\right)$ interacting with a 1-rarefaction front $\left(u_{l}, u_{m}\right)$. We remark that, as in Figure 9, the 2-shock is "on top" of the 1-rarefaction front in $(t, x)$ coordinates, because the first one evolves backward in time, while the latter evolves forward in time. Hence

$$
u_{r}=\Phi_{2}^{l}\left(\varepsilon_{2}, \Phi_{1}\left(\varepsilon_{1}, u_{l}\right)\right)
$$

with $\varepsilon_{1}>0$ and $\varepsilon_{2}<0$. We have: 
Lemma 13. Suppose that all the states considered are in $B\left(U_{0}^{-} ; r\right)$. There are some $\varepsilon_{1}^{\prime}$ and $\varepsilon_{2}^{\prime}$ such that

$$
u_{r}=\Phi_{1}\left(\varepsilon_{1}^{\prime}, \Phi_{2}^{l}\left(\varepsilon_{2}^{\prime}, u_{l}\right)\right)
$$

with

$$
\left|\varepsilon_{1}-\varepsilon_{1}^{\prime}\right|+\left|\varepsilon_{2}-\varepsilon_{2}^{\prime}\right|=O(1)\left|\varepsilon_{1}\right|\left|\varepsilon_{2}\right|
$$

Proof. This lemma is obtained exactly as Lax's theorem by the implicit function theorem, noting that

$$
\frac{d}{d \varepsilon} \Phi_{2}^{l}(\cdot, \omega)_{\mid \varepsilon=0}=-r_{2}(\omega)
$$

We fix $\tilde{u}_{m}:=\Phi_{2}^{l}\left(\varepsilon_{2}^{\prime}, u_{l}\right)$. The approximation $U^{n}$ is extended over the interaction point by $u_{l}, \tilde{u}_{m}$ and $u_{r}$ with (see Figure 9 :

- $u_{l}$ and $\tilde{u}_{m}$ separated by a backward-in-time 2-front at shock speed,

- $\tilde{u}_{m}$ and $u_{r}$ separated by a forward-in-time 1-front at shock speed.

Note that if all the states are in $B\left(U_{0}^{-} ; r\right)$ and $r$ is small enough, then the front $\left(\tilde{u}_{m}, u_{r}\right)$ is a 1-rarefaction front, and $\left(\tilde{u}_{m}, u_{l}\right)$ is a 2-shock (see $[162)$ ).

In the above construction, the finite number of waves and interaction points is a consequence of the fact that fronts of the same family do not meet. So in order to prove that the above algorithm is well-defined, we only have to prove that all states stay in $B\left(U_{0}^{-} ; r\right)$.

\subsection{BV estimates and well-posedness of the algorithm}

We first establish estimates on the piecewise constant function $\hat{u}^{n}$ constructed after the first step of the algorithm. Then we look for estimates on $U^{n}$.

a. Estimates on $\hat{u}^{n}$. The $B V$ estimate on $\hat{u}^{n}$ is obtained as in the Eulerian case, and is even simpler, because the "fronts" that go out of an interaction with the strong 2-shock do not interact since they are all horizontal. We infer that there are only a finite number of fronts and interaction points analogously as in Section 3 . We omit the details.

In particular, if $T V\left(u_{0}\right)<\varepsilon_{0}$, then all the states under consideration on the right of the strong 2-shock are in $B\left(\bar{u}_{0} ; r\right)$.

Moreover, there is some constant $C>0$ such that

$$
\sum_{\substack{\alpha \text { meets } S \\ \text { at time } \tau}}\left|\sigma_{\alpha}\left(\tau^{-}\right)\right| \leq C \cdot T V\left(u_{0}\right) .
$$

Indeed (see for instance [8]), we consider an increasing sequence of piecewise affine space-like curves $\Gamma_{0}, \ldots, \Gamma_{N}$ such that: the curves $\Gamma_{i}$ are under $S, \Gamma_{0}$ starts at $\left(0,0^{+}\right)$and there is no interaction point between $\Gamma_{0}$ and $\{0\} \times[0,1]$, there is exactly one interaction point between $\Gamma_{i}$ and $\Gamma_{i+1}$ (including the "front/boundary" interaction points), and there is no interaction point between $\Gamma_{N}$ and $S$. See Figure 10 . 


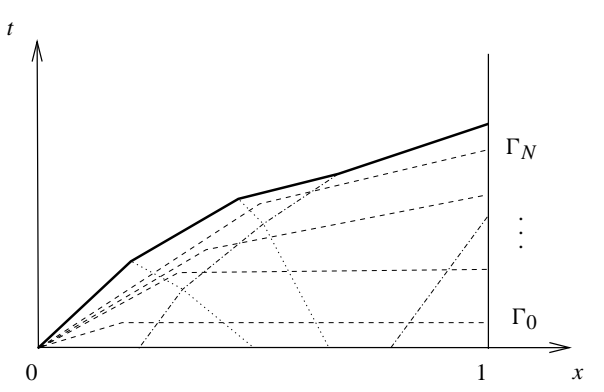

Fig. 10. $\Gamma_{0}, \ldots, \Gamma_{N}$.

Then we fix

$$
V_{i}:=\sum_{\sigma \text { intersects } \Gamma_{i}}|\sigma| \text { and } Q_{i}:=\sum_{\substack{\sigma, \sigma^{\prime} \text { approaching } \\ \text { and intersecting } \Gamma_{i}}}|\sigma|\left|\sigma^{\prime}\right| .
$$

Then one gets $V_{i}+C Q_{i}$ decreasing for suitable $C$, which yields 163 .

Finally, as in the Eulerian case, we get: for any rarefaction front $\sigma$ (that one can trace beyond interactions because of the convention that we made),

$$
|\sigma(t)| \leq C|\sigma(0)|=O(1 / n),
$$

for $t$ a time before interaction with the strong 2-shock.

b. Estimates on $U^{n}$. From (163), (151), (153) and Schochet's lemma (the transmission matrix is bounded when the states on the sides of the shock lie in $\mathfrak{D}$ ), we get

$$
\sum_{\sigma \text { entering } \Omega}|\sigma| \leq C^{\prime} \cdot T V\left(u_{0}\right)
$$

where the fronts considered are those in $U^{n}$; also for any rarefaction front that extends a rarefaction front interacting with the strong 2 -shock,

$$
\left|\sigma\left(t^{+}\right)\right|=O(1)\left|\sigma\left(t^{-}\right)\right| \leq C|\sigma(0)|=O(1 / n),
$$

where $\left|\sigma\left(t^{+}\right)\right|$(resp. $\left|\sigma\left(t^{-}\right)\right|$) is the value of the strength of $\sigma$ after (resp. before) interaction with the strong shock. Of course, for "new" rarefaction fronts (that come from the interaction of a weak 2-shock with the strong 2-shock), one has $\sigma\left(\tau^{+}\right) \leq 1 / n$.

For $x \in[0,1]$, we introduce the curve $\Xi(x)$ as the union of $[\mathfrak{S}(x),+\infty) \times\{x\}$ and of the part of $\mathfrak{S}$ from $\mathfrak{S}(0)$ to $\mathfrak{S}(x)$ (see Figure 11(a)). Also, let $\mathcal{A}(x)$ be the set of all couples $\left(\sigma, \sigma^{\prime}\right)$ intersecting $\Xi(x)$ and approaching (that is, $\sigma$ is a 1-front and $\sigma^{\prime}$ is a 2-front, with $\sigma^{\prime}$ above $\sigma$, that is, in that case, located later in time).

Then we can define

$$
\mathcal{V}(x):=\sum_{\sigma \text { intersects } \Xi(x)}|\sigma| \text { and } \mathcal{Q}(x):=\sum_{\left(\sigma, \sigma^{\prime}\right) \in \mathcal{A}(x)}|\sigma|\left|\sigma^{\prime}\right|,
$$


Then again, for suitable $C_{0}, \mathcal{V}(x)+C_{0} \mathcal{Q}(x)$ decreases as $1-x$ increases. In particular, using (165), we have the estimate

$$
\mathcal{V}(x=0) \leq O(1) T V\left(u_{0}\right)
$$

Note in particular that $\mathcal{V}(x=0)$ dominates $T V_{\mathbb{R}^{+}}\left(U_{\mid \mathbb{R}^{+} \times\{0\}}^{n}\right)$.

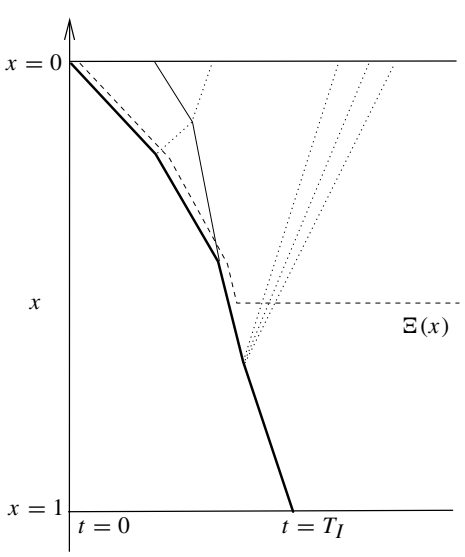

(a) The curve $\Xi(x)$

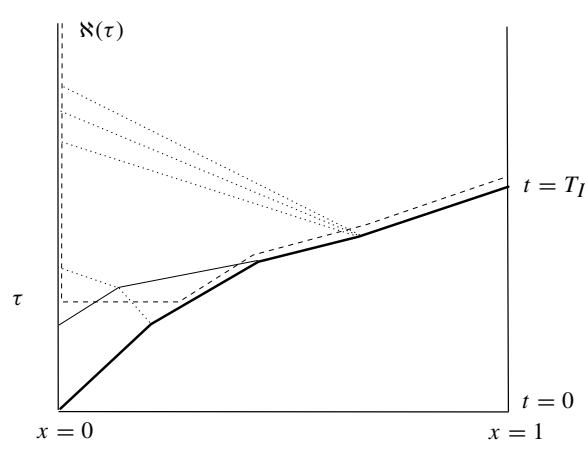

(b) The curve $\aleph(\tau)$

Fig. 11. Two families of curves.

Now, we introduce a family of curves depending on the time $\tau$, as described in Figure 11 b): the curve $\aleph(\tau)$ is composed of $[\tau,+\infty) \times\{0\}$, the horizontal line from $(\tau, 0)$ to the point $\left(\tau, \mathfrak{S}^{-1}(\tau)\right)$ (if $\mathfrak{S}^{-1}(\tau)$ is defined, and $(\tau, 1)$ otherwise), and then the curve following $\mathfrak{S}$ (along its left side) to the right boundary of the domain.

As previously, we define $\mathcal{V}$ and $\mathcal{Q}$ along the curve $\aleph(\tau)$. Note that by what precedes, $V$ computed on $\aleph(0)$ is of order $O(1) T V\left(u_{0}\right)$. Then one easily sees that $\mathcal{V}+C \mathcal{Q}$ computed along $\aleph(\tau)$ is nonincreasing, which leads to

$$
T V_{\{t\} \times[0, S(t))}\left(U^{n}\right) \leq C^{\prime \prime} T V\left(u_{0}\right)
$$

for any $t$ and suitable $C^{\prime \prime}$. This implies that the states considered are in the domain described in Subsection 6.2 if $T V\left(u_{0}\right)$ is small enough. All the same, we also conclude that all rarefaction fronts satisfy

$$
|\sigma|=O(1 / n)
$$

by distinguishing the rarefaction fronts that come from a rarefaction front "crossing" the strong shock, and rarefaction fronts that come from the interaction of the strong 2-shock with another 2 -shock (the latter have original strength of order $1 / n$ when leaving the strong 2-shock). We omit the details. 


\subsection{Conclusion}

As previously, we also deduce a $\operatorname{Lip}_{t}\left(L_{x}^{1}\right)$ bound on $U^{n}$, and hence, up to a subsequence, one gets

$$
U^{n} \rightarrow U \quad \text { in } L_{\text {loc }}^{1}([0,+\infty) \times[0,1]) .
$$

The fact that the limit is an entropic solution of $(\mathrm{P})$ is deduced as previously, using (164) and (167): all fronts travel approximately at shock speed, and the rarefaction fronts are all of size $O(1 / n)$.

It remains to justify that $U$ reaches a constant state. It is sufficient to prove that

$$
U^{n}(t, \cdot) \text { is constant for } t \geq 1 .
$$

But this is a consequence of the construction which implies that there are only 1-rarefaction fronts in the domain for $t \geq T_{i}$. This can be seen as follows. The fronts above the strong 2-shock come from a point $\left(t_{i}, x_{i}\right)$. These fronts are only 1-rarefaction fronts or shock 2-fronts (and each $i$-front keeps its nature-rarefaction front or shock-after successive interactions). But the 2-shocks evolve backward in time. Consequently, for $t \geq T_{i}$, only 1-rarefactions fronts can be left in the domain. But rarefaction fronts going forward in time do not interact. Using the definition of $r$ (characteristic speeds are bounded away from 0), we deduce (168). This ends the proof of Proposition 5

Now, in order to finish the proof of Theorem 2, it remains to establish corresponding Propositions 3 and 4 for $(\mathrm{P})$. Proposition 3 for $(\mathrm{P})$ is simpler to prove than for $(\mathrm{EI})$, because there is only one zone in which $\lambda_{1}<0<\lambda_{2}$. We omit the details. Then the proof of Proposition 4 for the system $(\mathrm{P})$ is similar to the one for $(\mathrm{EI})$, with both strong shocks to be retained. This is left to the reader.

Acknowledgments. The author wishes to thank the referee for stimulating remarks on the first version of the paper.

\section{References}

[1] Alber, H.-D.: Local existence of weak solutions to the quasilinear wave equation for large initial values. Math. Z. 190, 249-276 (1985) Zbl 0581.35049 MR 0797541

[2] Amadori, D., Colombo, R. M.: Continuous dependence for $2 \times 2$ conservation laws with boundary, J. Differential Equations 138, 229-266 (1997) Zbl 0884.35091 MR 1462268

[3] Ancona, F., Bressan, A., Coclite, G. M.: Some results on the boundary control of systems of conservation laws. In: Hyperbolic Problems: Theory, Numerics, Applications, Springer, Berlin, 255-264 (2003) Zbl 1073.93029 MR 2053176

[4] Ancona, F., Coclite, G. M.: On the attainable set for Temple class systems with boundary controls. SIAM J. Control Optim. 43, 2166-2190 (2005) Zbl 1087.93010 MR 2179483

[5] Ancona, F., Marson, A.: On the attainable set for scalar nonlinear conservation laws with boundary control. SIAM J. Control Optim. 36, 290-312 (1998) Zbl 0919.35082 MR 1616586

[6] Beauchard, K.: Local controllability of a 1-D Schrödinger equation. J. Math. Pures Appl. 84, 851-956 (2005) Zbl pre02228731 MR 2144647 
[7] Beauchard, K., Coron, J.-M.: Controllability of a quantum particle in a moving potential well. J. Funct. Anal. 232, 328-389 (2006) Zbl pre05017416 MR 220740

[8] Bressan, A.: Hyperbolic Systems of Conservation Laws, the One-Dimensional Cauchy Problem. Oxford Lecture Ser. Math. Appl. 20, Oxford Univ. Press (2000) Zbl 0997.35002 MR 1816648

[9] Bressan, A., Coclite, G. M.: On the boundary control of systems of conservation laws. SIAM J. Control Optim. 41, 607-622 (2002) Zbl 1026.35075 MR 1920513

[10] Bressan, A., Colombo, R. M.: Unique solutions of $2 \times 2$ conservation laws with large data. Indiana Univ. Math. J. 44, 677-725 (1995) Zbl 0852.35092 MR 1375345

[11] Chang, T., Hsiao L.: The Riemann Problem and Interaction of Waves in Gas Dynamics. Pitman Monogr. Surveys Pure Appl. Math. 41, Longman (1989) Zbl 0698.76078 MR 0994414

[12] Chen, Q. G., Dehua, W.: The Cauchy problem for the Euler equations for compressible fluids. In: Handbook of Mathematical Fluid Dynamics. Vol. I, S. Friedlander and D. Serre (eds.), North-Holland, Amsterdam, 421-543 (2002) Zbl pre01942876 MR 1942468

[13] Corli, A., Sablé-Tougeron, M.: Perturbations of bounded variation of a strong shock wave. J. Differential Equations 138, 195-228 (1997) Zbl 0881.35071 MR 1462267

[14] Coron, J.-M.: Global Asymptotic stabilization for controllable systems without drift. Math. Control Signal Systems 5, 295-312 (1992) Zbl 0760.93067| MR 1164379

[15] Coron, J.-M.: On the controllability of 2-D incompressible perfect fluids. J. Math. Pures Appl. 75, 155-188 (1996) Zbl 0848.76013 MR 1380673

[16] Coron, J.-M.: Local controllability of a 1-D tank containing a fluid modeled by the shallow water equations. ESAIM Control Optim. Calc. Var. 8, 513-554 (2002) Zbl 1071.76012 MR 1932962

[17] Di Perna, R. J.: Global solutions to a class of nonlinear hyperbolic systems of equations. Comm. Pure Appl. Math. 26, 1-28 (1973) Zbl 0256.35053 MR 0330788

[18] Dubois, F., LeFloch, P. G.: Boundary conditions for nonlinear hyperbolic systems of conservation laws. J. Differential Equations 71, 93-122 (1988) Zbl 0649.35057 MR 0922200

[19] Glass, O.: Exact boundary controllability of 3-D Euler equation, ESAIM Control Optim. Calc. Var. 5, 1-44 (2000) Zbl 0940.93012 MR 1745685

[20] Glass, O.: On the controllability of the Vlasov-Poisson system. J. Differential Equations 195, 332-379 (2003) Zbl pre02032993 MR 2016816

[21] Glimm, J.: Solutions in the large for nonlinear hyperbolic systems of equations. Comm. Pure Appl. Math. 18, 697-715 (1965) Zbl 0141.28902 MR 0194770

[22] Glimm, J., Lax, P. D.: Decay of solutions of systems of nonlinear hyperbolic conservation laws. Mem. Amer. Math. Soc. 101 (1970) Zbl 0204.11304 MR 0265767

[23] Gugat, M., Leugering, G.: Global boundary controllability of the de St. Venant equations between steady states. Ann. Inst. H. Poincaré Anal. Non Linéaire 20, 1-11 (2003) Zbl 1032.93030 MR 1958159

[24] Horsin, T.: On the controllability of the Burgers equation. ESAIM Control Optim. Calc. Var. 3, 83-95 (1998) Zbl 0897.93034 MR 1612027

[25] Lax, P. D.: Hyperbolic systems of conservation laws. Comm. Pure Appl. Math. 10, 537-566 (1957) Zbl 0081.08803 MR 0093653

[26] Lax, P. D.: Hyperbolic Systems of Conservation Laws and the Mathematical Theory of Shock Waves. CBMS Reg. Conf. Ser. Appl. Math. 11, SIAM, Philadelphia (1973) Zbl 0268.35062 MR 0350216

[27] Lewicka, M., Trivisa, K.: On the $L^{1}$ well posedness of systems of conservation laws near solutions containing two large shocks. J. Differential Equations 179, 133-177 (2002) Zbl 0994.35086 MR 1883740 
[28] Li, T.-T., Rao, B.-P.: Exact boundary controllability for quasi-linear hyperbolic systems. SIAM J. Control Optim. 41, 1748-1755 (2003) Zbl 1032.35124 MR 1972532

[29] Lions, P.-L., Perthame, B., Souganidis, P. E.: Existence and stability of entropy solutions for the hyperbolic systems of isentropic gas dynamics in Eulerian and Lagrangian coordinates. Comm. Pure Appl. Math. 49, 599-638 (1996) Zbl 0853.76077 MR 1383202

[30] Majda, A.: Compressible Fluid Flow and Systems of Conservation Laws in Several Space Variables. Springer, New York (1984) Zbl 0537.76001 MR 0748308

[31] Sablé-Tougeron, M.: Stabilité de la structure d'une solution de Riemann à deux grands chocs. Ann. Univ. Ferrara Sez. VII (N.S.) 44, 129-172 (1998) Zbl 0943.35052 MR 1744132

[32] Schochet, S.: Sufficient conditions for local existence via Glimm's scheme for large BV data. J. Differential Equations 89, 317-354 (1991) Zbl 0733.35072 MR 1091481

[33] Serre, D.: Systems of Conservation Laws. I: Hyperbolicity, Entropies, Shock Waves. Cambridge Univ. Press, Cambridge (1999) Zbl 0930.35001 MR 1707279

[34] Smoller, J.: Shock Waves and Reaction-Diffusion Equations. 2nd ed., Grundlehren Math. Wiss. 258, Springer, New York (1994) Zbl 0807.35002 MR 1301779

[35] Wagner, D.: Equivalence of the Euler and Lagrangian equations of gas dynamics for weak solutions. J. Differential Equations 68, 118-136 (1987) Zbl 0647.76049 MR 0885816 